\title{
WestVirginiaUniversity
}

THE RESEARCH REPOSITORY @ WVU

Graduate Theses, Dissertations, and Problem Reports

2013

\section{The Diabetic Heart: Examination of the Inner Mitochondrial Membrane}

Tara Lyne Slider Croston

West Virginia University

Follow this and additional works at: https://researchrepository.wvu.edu/etd

\section{Recommended Citation}

Croston, Tara Lyne Slider, "The Diabetic Heart: Examination of the Inner Mitochondrial Membrane" (2013). Graduate Theses, Dissertations, and Problem Reports. 4958.

https://researchrepository.wvu.edu/etd/4958

This Dissertation is protected by copyright and/or related rights. It has been brought to you by the The Research Repository @ WVU with permission from the rights-holder(s). You are free to use this Dissertation in any way that is permitted by the copyright and related rights legislation that applies to your use. For other uses you must obtain permission from the rights-holder(s) directly, unless additional rights are indicated by a Creative Commons license in the record and/ or on the work itself. This Dissertation has been accepted for inclusion in WVU Graduate Theses, Dissertations, and Problem Reports collection by an authorized administrator of The Research Repository @ WVU.

For more information, please contact researchrepository@mail.wvu.edu. 


\title{
The Diabetic Heart:
}

\section{Examination of the Inner Mitochondrial Membrane}

\author{
Tara Lyne Slider Croston
}

\begin{abstract}
Dissertation submitted to the School of Medicine at West Virginia University in partial fulfillment of the requirements for the degree of
\end{abstract}

\author{
Doctor of Philosophy \\ in \\ Exercise Physiology \\ John M. Hollander, Ph.D., Chair \\ Stephen E. Alway, Ph.D. \\ Emidio Pistilli, Ph.D. \\ I. Mark Olfert, Ph.D. \\ S. Jamal Mustafa, Ph.D. \\ Division of Exercise Physiology \\ Morgantown, West Virginia \\ 2013
}

Keywords: Diabetes mellitus, Mitochondria, Cardiolipin, Grp75, Human heart 


\section{ABSTRACT}

\section{The Diabetic Heart: Examination of the Inner Mitochondrial Membrane}

\section{Tara L. Croston}

Cardiovascular complications, such as diabetic cardiomyopathy, are the primary cause of morbidity and mortality in patients with diabetes mellitus. The hyperglycemic environment associated with diabetes mellitus has been shown to induce the mitochondrial dysfunction contributing to diabetic cardiomyopathy. The examination of mitochondrial function is further complicated by the existence of two spatially and functionally distinct cardiac mitochondrial subpopulations, the subsarcolemmal (SSM) interfibrillar (IFM) mitochondria. These two pools of mitochondria respond differently to pathologic stimuli, such as diabetes mellitus. Targeted by the deleterious effects of diabetes mellitus, the inner mitochondrial membrane (IMM) is composed of a specific lipid environment that houses many critical processes and machinery required for proper mitochondrial function. The impact of diabetes mellitus on the IMM, including the lipids and processes housed within, is not completely understood in type 1 diabetic setting, and has not been assessed in mitochondrial subpopulations from type 2 diabetic human heart. The goal of the studies included in this dissertation was to understand the importance of IMM preservation during diabetes mellitus and to translate rodent mitochondrial dysfunction that occurs during diabetic cardiomyopathy to the human diabetic heart. FVB mice (20-25 grams) were made type 1 diabetic through multiple low dose injections of streptozotocin (STZ) for five consecutive days. Five weeks post diabetic onset, hearts were excised and mitochondrial subpopulations were isolated. For the type 2 diabetic human population, right atrial appendages were excised during coronary artery bypass grafting or valve replacement surgery, from which mitochondrial subpopulations were isolated. Cardiolipin, an essential phospholipid contained within the IMM, was shown to be decreased during a type 1 diabetic insult; however the mechanism attributing to this decrease in unknown; therefore, the cardiolipin biosynthetic pathway was evaluated in the type 1 diabetic mouse model. Results indicated decreased cardiolipin synthase protein content and activity in the diabetic IFM. The data also revealed a decrease in two critical cardiolipin-ATP synthase associations, which could potentially contribute to the observed decreased ATP synthase activity in the diabetic IFM, all of which are located in the IMM. Within the type 1 diabetic mouse model, a key constituent involved in protein import, glucose regulated protein 75 , has been reported to be decreased and when overexpressed, preliminary data depicted mitochondrial functional restoration in the diabetic IFM. Mitochondrial dysfunction, as evidenced by decreased state 3 mitochondrial respiration, as well as decreased electron transport chain complex I and complex IV activity was observed in the SSM of type 2 diabetic human heart, compared to the non-diabetic heart. These results suggest for the first time, that mitochondrial dysfunction is present in the type 2 diabetic human heart and that SSM impacted to a greater extent than IFM. Taken together, the results of these studies demonstrate the importance of preserving the IMM structure to alleviate mitochondrial dysfunction associated with the diabetic heart, as well as understanding the effect of diabetes mellitus on human cardiac mitochondrial 
subpopulations. Further, the preliminary results highlight therapeutic strategies involving protein import constituents on the ability to attenuate mitochondrial dysfunction that occurred during a diabetic insult. 


\section{ACKNOWLEDGEMENTS}

First, I would like to acknowledge my advisor and mentor, Dr. John Hollander. Thank you for offering me the opportunity to become a member of your laboratory, where I was able to grow both personally and professionally. The training and confidence you have instilled in me will guide me to thrive in the next phases of my career and of my life. Thank you for your honesty, guidance, and time you have devoted to me over the past five years. I would also like to acknowledge my committee members, Dr. Stephen Alway, Dr. Emidio Pistilli, Dr. Mark Olfert, and Dr. S. Jamal Mustafa. Thank you for not only finding the time to meet with me, but for your encouragement and direction during my graduate career.

I would also like to thank the members in my laboratory, especially Dr. Erinne Dabkowski for the initial training of my career, as well as your continued support through my time at West Virginia University. I would like to thank Courtney Williamson, Dr. Walter Baseler, Danielle Shepherd, Cody Nichols, Dr. Rajaganapathi Jagannathan and Sara Lewis for all of their help and guidance over these past few years. I would also like thank honorary lab member Gerald Audet and Janelle Stricker for their encouragement and help. Lastly, I want to especially thank Dharendra Thapa for all your help and encouragement, as well as for being there from the beginning of my time in Dr. Hollander's laboratory.

I would like to acknowledge the Exercise Physiology and Graduate Education Department, especially Dr. Fred Minnear at West Virginia University for the support during my graduate career. I would like to thank Dr. Jamal Mustafa, Dr. Richard Dey and the Cardiovascular and Pulmonary T32 committee for selecting me to have an appointment on this distinguished training grant.

Finally, I want to thank my family. Thank you for your steadfast support, unwavering devotion and confidence during my graduate career. I could not have asked for more. To my husband Garrett, you are my rock and for that, I am eternally grateful. 


\section{LIST OF ABBREVIATIONS}

ADP

ATP

ATP5A

ATP5B

ATP5F1

ATP5H

BAX

BSA

CAD

CDP-DAG

CDS

CL

cox

CRLS

DCCT

ETC

FSC

GLUT2

GLUT4

GFP

Grp75
Adenosine Diphosphate

Adenosine Triphosphate

ATP Synthase Alpha

ATP Synthase Beta

ATP Synthase subunit b

ATP Synthase subunit d

B-cell lymphoma-2-associated $X$ protein

Bovine Serum Albumin

Coronary Artery Disease

Cytidine diphosphate diacylglycerol

Cytidine diphosphate diacylglycerol synthase

Cardiolipin

Cytochrome C Oxidase

Cardiolipin synthase

Diabetes Control and Complications Trial

Electron Transport Chain

Forward Scatter

Glucose Transporter 2

Glucose Transporter 4

Green Fluorescent Protein

Glucose Regulated Protein 75 


$\begin{array}{ll}\text { HbA1C } & \text { Glycated hemoglobin } \\ \text { HSP } & \text { Heat Shock Protein } \\ \text { IFM } & \text { Interfibrillar Mitochondria } \\ \text { IMM (or IM) } & \text { Inner Mitochondrial Membrane } \\ \text { IMS } & \text { Inner Mitochondrial Space } \\ \text { mMDH2 } & \text { malate dehydrogenase } \\ \text { MHC } & \text { Myosin Heavy Chain } \\ \text { MPP } & \text { Matrix-processing peptidase } \\ \text { MPTP } & \text { Mitochondrial permeability transition pore } \\ \text { NDUF3 } & \text { NADH dehydrogenase [ubiquinone] 1 alpha } \\ \text { NGSP } & \text { National Glycohemoglobin Standardization Program } \\ \text { OGT } & \text { Oral Glucose Tolerance } \\ \text { OMM (or OM) } & \text { Outer Mitochondrial Membrane } \\ \text { OXPHOS } & \text { Oxidative Phosphorylation } \\ \text { PAGE } & \text { Polyacrylamide Gel Electrophoresis } \\ \text { PAM } & \text { Presequence Translocase-associated Motor } \\ \text { PBS } & \text { Phosphate Buffered Saline } \\ \text { PCR } & \text { Phospherase Chain Reaction } \\ \text { PG } & \begin{array}{l}\text { PGos } \\ \text { PGP }\end{array}\end{array}$


SSM

STZ

TG

TIM

TOM

VDAC

WT

ZDF
Subsarcolemmal Mitochondria

Streptozotocin

Transgenic

Translocase of the Inner Membrane

Translocase of the Outer Membrane

Voltage Dependent Anion Channel

Wild Type

Zucker Diabetic Fatty Rat 


\section{TABLE OF CONTENTS}

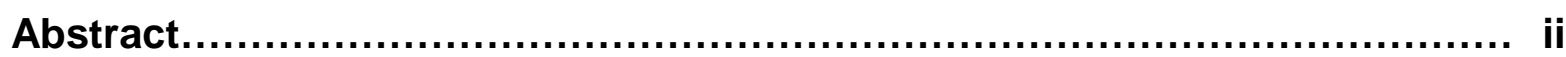

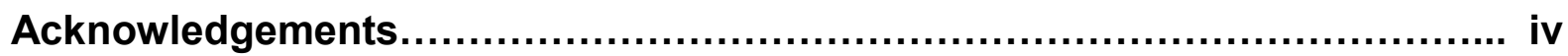

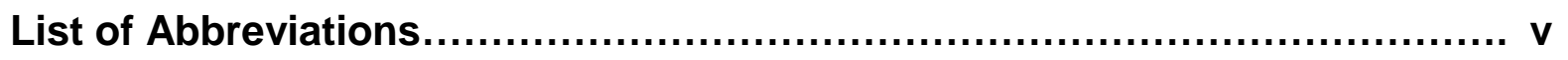

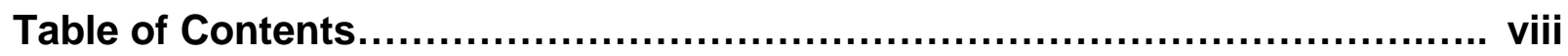

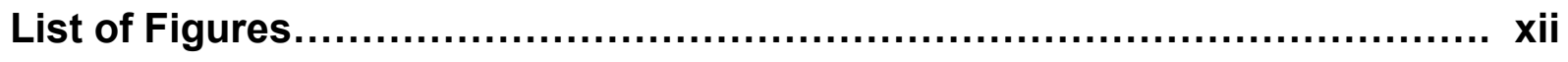

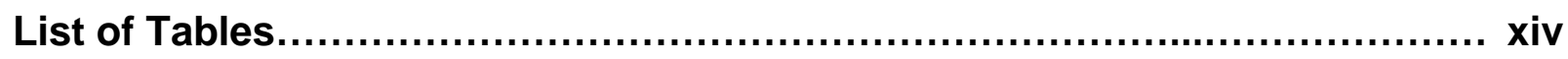

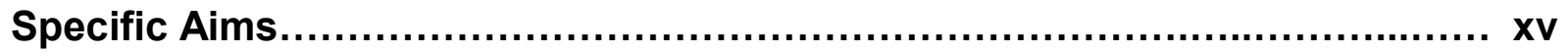

Chapter 1. Background and Significance...................................... 1

1.1 Diabetes Mellitus............................................ 2

1.1.1 Type 1 diabetes mellitus.............................. 3

1.1.2 Type 2 diabetes mellitus $\ldots \ldots \ldots \ldots \ldots \ldots \ldots \ldots \ldots \ldots \ldots \ldots \ldots, 4$

1.1 .3 Significance....................................... 5

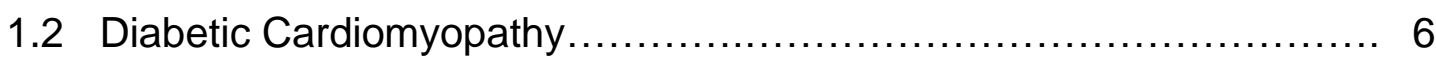

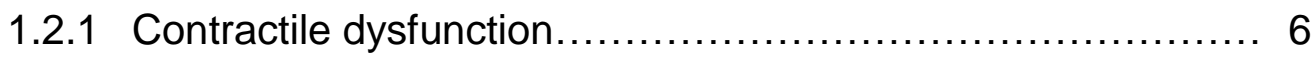

1.2.2 Cardiac metabolism................................... 8

1.2 .3 Oxidative stress $\ldots \ldots \ldots \ldots \ldots \ldots \ldots \ldots \ldots \ldots \ldots \ldots \ldots \ldots \ldots . \ldots$

1.3 Mouse models of diabetic cardiomyopathy....................... 11 
1.4 Mitochondrial Subpopulations

1.5 Mitochondrial Dysfunction in the diabetic heart........................ 16

1.5.1 Oxidative stress............................................. 17

1.5.2 Calcium homeostasis..................................... 17

1.5.3 Lipotoxicity................................................ 18

1.5.4 Mitochondrial dysfunction in the type 1 diabetic heart.......... 19

1.5.5 Mitochondrial dysfunction in the type 2 diabetic heart.......... 19

1.6 Inner Mitochondrial Membrane ....................................... 20

1.6.1 Inner mitochondrial membrane machinery..................... 21

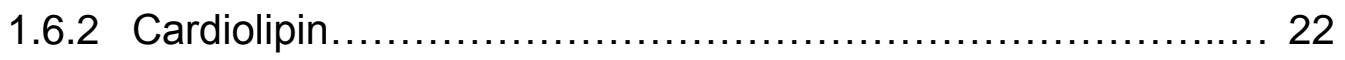

$1.7 \quad$ Mitochondrial Protein Import........................................ 25

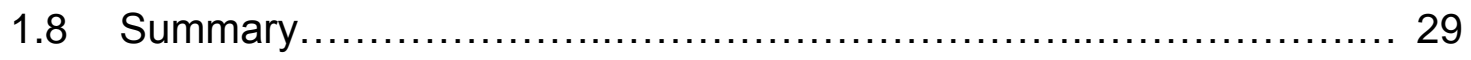

1.9 References........................................................ 30

\section{Chapter 2. Evaluation of the cardiolipin biosynthetic pathway and its}

interactions in the diabetic heart................................................... 53

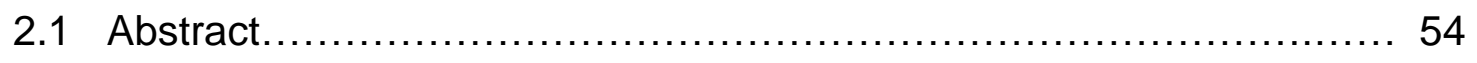

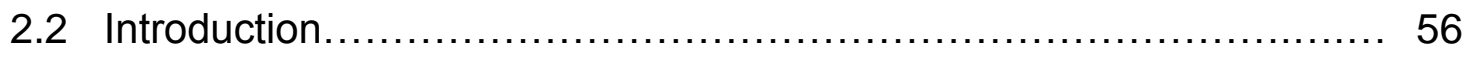


2.3 Methods............................................................... 59

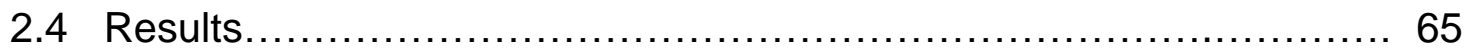

2.5 Discussion............................................................. 70

2.6 Acknowledgements ................................................ 77

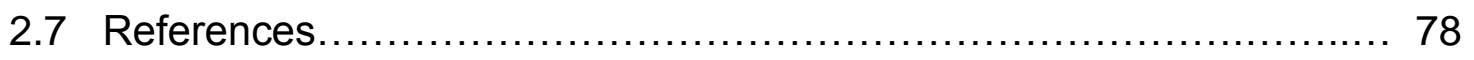

2.8 Figures and figure legends......................................... 83

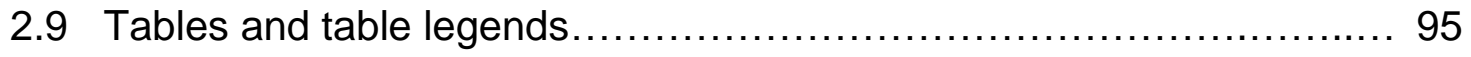

Chapter 3. Mitochondrial Dysfunction in the Human Diabetic Heart: Impact on Distinct Mitochondrial Subpopulations........................................ 97

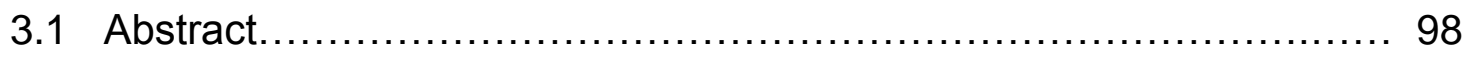

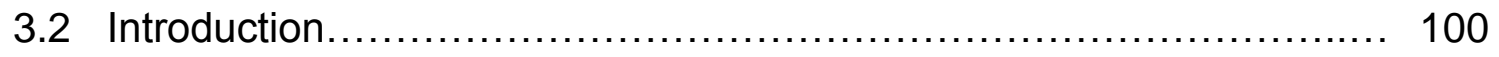

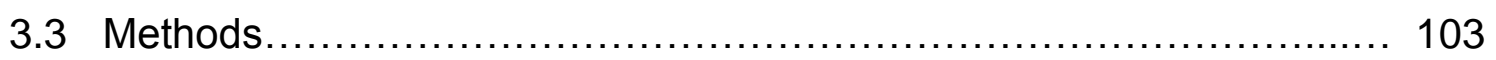

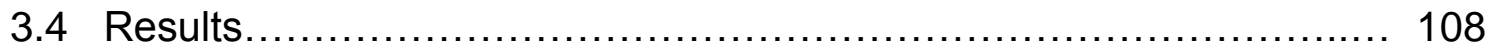

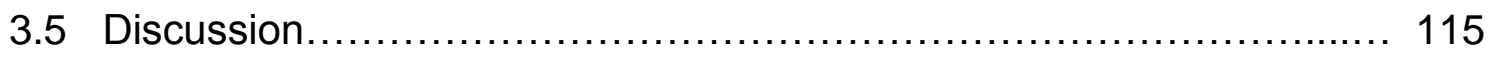

3.6 Acknowledgements ............................................... 120

3.7 References......................................................... 121

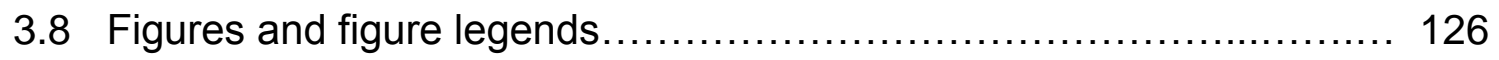

3.9 Tables and table legends............................................. 144

Chapter 4. General Discussion.................................................... 152

4.1 References............................................................. 166 


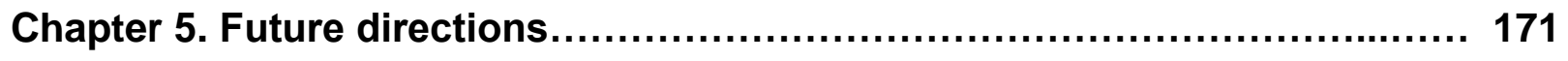

5.1 References.......................................................... 177

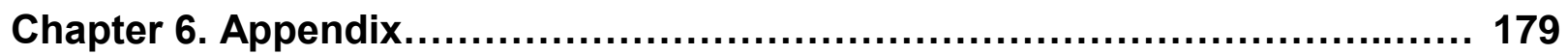

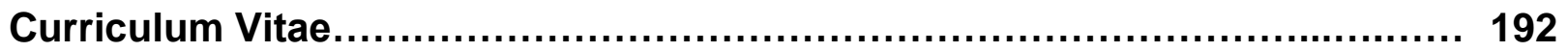




\section{LIST OF FIGURES}

\section{Chapter 1}

1.1 Mitochondrial Subpopulations ........................................ 15

1.2 Schematic of the inner mitochondrial membrane ......................... 21

1.3 Structure of tetralinoleoyl cardiolipin ..................................... 23

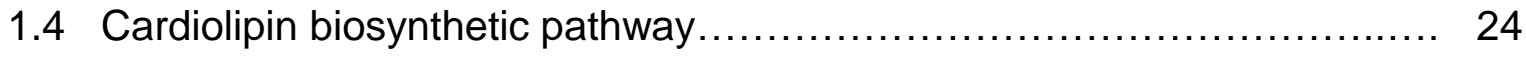

1.5 Mitochondrial import of matrix-targeted proteins ......................... 26

1.6 MitoGFP1 construct and protein import................................ 28

\section{Chapter 2}

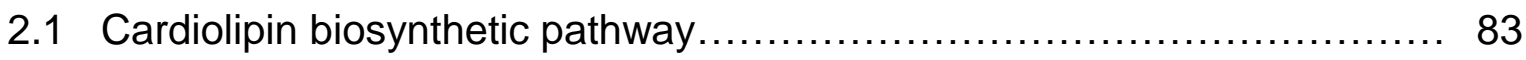

2.2 Protein content of cardiolipin biosynthetic pathway constituents........... 85

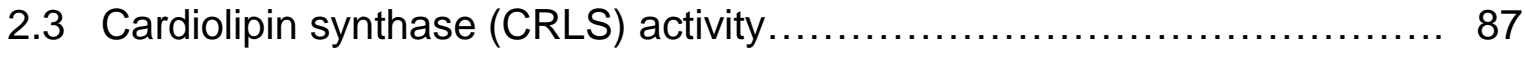

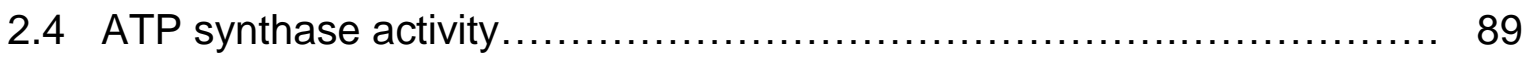

2.5 Cardiolipin - ATP synthase association .................................. 91

2.6 Native cardiolipin - ATP synthase association........................... 93

\section{Chapter 3}

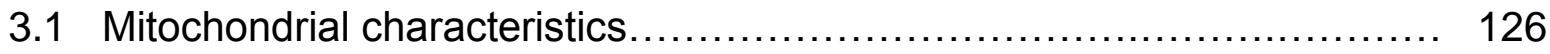

3.2 Mitochondrial subpopulation respiration rates.......................... 128

3.3 Linear spline illustrations for mitochondrial respiration................... 130

3.4 Electron transport chain activities in mitochondrial subpopulations......... 133 
3.5 Linear spline illustrations for electron transport chain complex

3.6 Electron transport chain complex I expression $\ldots \ldots \ldots \ldots \ldots \ldots \ldots \ldots \ldots \ldots \ldots \ldots \ldots \ldots \ldots$

3.7 Electron transport chain complex IV expression...................... 140

3.8 Linear spline illustration for state 3 mitochondrial respiration and ETC complex I activity identifying BMI levels

\section{Chapter 4}

4.1 Mitochondrial proteome during different diabetic states

\section{Chapter 5}

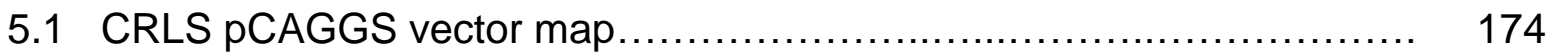

\section{Chapter 6}

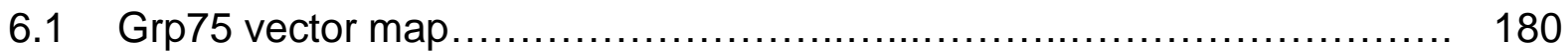

6.2 The protective benefit of Grp75 overexpression on mitochondrial

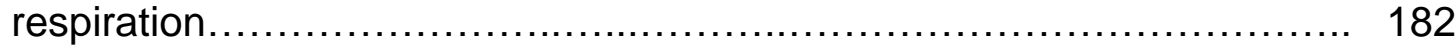

6.3 Protein levels of Grp75 in the type 2 diabetic human heart.............. 184

6.4 Preliminary protein import in the type 2 diabetic human heart............ 186

6.5 Cardiolipin content in the type 2 diabetic heart........................ 188 


\section{LIST OF TABLES}

\section{Chapter 1}

1.1 American Diabetes Association: Criteria for the diagnosis of diabetes..... 2

\section{Chapter 2}

2.1 mRNA analysis of inner mitochondrial membrane proteins 95

\section{Chapter 3}

3.1 Patient Demographics and Characteristics.

3.2 Non-diabetic and Diabetic Patients with Both Coronary Artery Diseases and Hypertension

3.3 Non-Coronary Artery Diseases (CAD) and CAD Patients without Diabetes Mellitus

3.4 Non-Hypertensive (HT) and Hypertensive Patients without Diabetes Mellitus

\section{Chapter 4}

4.1 Mitochondrial dysfunction associated with type 1 and type 2 diabetes mellitus in mouse and human models

\section{Chapter 6}

6.1 Grp75 overexpression in isolated mitochondrial subpopulations 190 


\section{SPECIFIC AIMS}

Cardiovascular complications, such as diabetic cardiomyopathy, are the primary cause of heart failure in patients with diabetes mellitus (3). The hyperglycemic environment produced during diabetes mellitus contributes to diabetic-associated mitochondrial dysfunction $(8,9)$. A critical component in the mitochondrion is the inner mitochondrial membrane (IMM) which contains a signature lipid environment required for proper mitochondrial function. Cardiac mitochondria consist of two spatially distinct subpopulations: subsarcolemmal mitochondria (SSM) and interfibrillar mitochondria (IFM), of which no group has currently examined in the diabetic human heart.

Cardiolipin, an integral phospholipid located in the IMM, has been shown to play a vital role in mitochondrial bioenergetics (1). We have previously observed decreased cardiolipin content during a type 1 diabetic insult (6), specifically in IFM (5); however, the mechanisms underlying this loss are unknown. Proteomic data indicates decreased abundance of mitochondrial proteins in the diabetic heart, which include IMM proteins $(2,7)$, such as electron transport chain (ETC) proteins associated with cardiolipin. Our laboratory has suggested a correlation between the proteomic decrements to a decrease in nuclear-encoded protein import into the mitochondria during a type 1 diabetic insult (2). Mitochondrial glucose regulated protein 75 (Grp75), an essential component of nuclear-encoded protein import, was also decreased in diabetes mellitus

$(2,4)$. Currently, the mechanisms contributing to cardiolipin loss and the therapeutic benefit of molecular gene manipulation on a key mitochondrial protein import constituent during diabetes mellitus are currently unclear. 
Our long term goal is to understand the importance of IMM preservation during diabetes mellitus and to translate rodent mitochondrial dysfunction that occurs during diabetic cardiomyopathy to the human diabetic heart. The objectives of this dissertation were to: (1) to determine how the cardiolipin biosynthesis pathway and cardiolipin-protein interactions are impacted by type 1 diabetes mellitus; (2) to determine the therapeutic value of mitochondrial Grp75 overexpression on mitochondrial protein import during a type 1 diabetic insult; and (3) to examine how mitochondrial subpopulations in the human heart are affected by diabetes mellitus. The central hypothesis of this dissertation was that the mitochondrion is negatively impacted by diabetes mellitus, leading to deleterious effects to the IMM and compromising the proteins associated with cardiolipin and protein import, which will translate to the diabetic human heart. The central hypothesis of this study will be examined by testing the following Specific Aims:

Specific Aim I: Determine the effect of a type 1 diabetic insult on the biosynthesis of cardiolipin, and evaluate key cardiolipin -protein interactions in the IMM. Further, determine if the effect is mitochondrial-subpopulation specific.

Our working hypothesis is that type 1 diabetes mellitus decreases cardiolipin content, due to deficient cardiolipin synthase, and that this lack of cardiolipin decreases its association with vital proteins in the IMM, specifically ATP synthase. Further, these effects will be most pronounced in IFM. 
Specific Aim II: Examine the therapeutic value of Grp75 overexpression on mitochondrial function following a type 1 diabetic insult. Further, determine if the effect is mitochondrial-subpopulation specific.

A novel transgenic mouse model created in our laboratory will be used to examine the role of Grp75 during diabetes mellitus. Our working hypothesis is that Grp75 overexpression will preserve mitochondrial function through a mechanism of enhanced protein import during a type 1 diabetic insult. These effects will be most pronounced in IFM.

Specific Aim III: Evaluate mitochondrial dysfunction in human heart tissue and examine the effects of diabetes mellitus on mitochondrial subpopulations.

Our working hypothesis is that diabetes mellitus causes abnormalities in mitochondria, characterized by decreased size, function and protein import. The decreased protein import potentially leads to mitochondrial proteome decrements and ultimately resulting in mitochondrial dysfunction associated with diabetes mellitus.

Our rationale for the research conducted for this dissertation is based on the importance of preserving the IMM structure to alleviate mitochondrial dysfunction associated with the diabetic heart, as well as understanding the effect of diabetes mellitus on human cardiac mitochondrial subpopulations. The anticipated outcomes were intended to highlight therapeutic strategies involving cardiolipin synthesis and protein import on the ability to attenuate mitochondrial dysfunction that occurred during a diabetic insult. The research gathered from this dissertation was innovative because 
the mechanisms elucidating mitochondrial dysfunction during a diabetic insult have not been previously examined, especially in a translational model, and several of the methodologies utilized were developed in our laboratory. At the completion of this dissertation, we were able to highlight potential at-risk targets within the IMM during a diabetic insult and determine the therapeutic benefit of overexpressing a key constituent in the protein import machinery. The results gathered has advanced our understanding of the mechanisms involved in both type 1 and type 2 models, from which therapeutic strategies can be developed. 


\section{REFERENCES}

1. Acehan D, Malhotra A, Xu Y, Ren M, Stokes DL, and Schlame M. Cardiolipin affects the supramolecular organization of ATP synthase in mitochondria. Biophys $J$ 100: 2184-2192, 2011.

2. Baseler WA, Dabkowski ER, Williamson CL, Croston TL, Thapa D, Powell MJ, Razunguzwa TT, and Hollander JM. Proteomic alterations of distinct mitochondrial subpopulations in the type 1 diabetic heart: contribution of protein import dysfunction. Am J Physiol Regul Integr Comp Physiol 300: R186-200, 2011.

3. Cai L, Li W, Wang G, Guo L, Jiang Y, and Kang YJ. Hyperglycemia-Induced Apoptosis in Mouse Myocardium. Diabetes 51: 1938-1948, 2002.

4. Dabkowski ER, Baseler WA, Williamson CL, Powell M, Razunguzwa TT, Frisbee JC, and Hollander JM. Mitochondrial dysfunction in the type 2 diabetic heart is associated with alterations in spatially distinct mitochondrial proteomes. Am J Physiol Heart Circ Physiol 299: H529-540, 2010.

5. Dabkowski ER, Williamson CL, Bukowski VC, Chapman RS, Leonard SS, Peer CJ, Callery PS, and Hollander JM. Diabetic cardiomyopathy-associated dysfunction in spatially distinct mitochondrial subpopulations. Am J Physiol Heart Circ Physiol 296: H359-369, 2009.

6. Han X, Yang J, Cheng H, Yang K, Abendschein DR, and Gross RW. Shotgun lipidomics identifies cardiolipin depletion in diabetic myocardium linking altered substrate utilization with mitochondrial dysfunction. Biochemistry 44: 16684-16694, 2005. 
7. Hollander JM, Baseler WA, and Dabkowski ER. Proteomic remodeling of mitochondria in heart failure. Congest Heart Fail 17: 262-268, 2011.

8. Rolo AP and Palmeira CM. Diabetes and mitochondrial function: Role of hyperglycemia and oxidative stress. Toxicology and Applied Pharmacology 212: 167178, 2006.

9. Shen X, Zheng S, Thongboonkerd V, Xu M, Pierce WM, Jr., Klein JB, and Epstein PN. Cardiac mitochondrial damage and biogenesis in a chronic model of type 1 diabetes. Am J Physiol Endocrinol Metab 287: E896-905, 2004. 


\section{Chapter 1:}

\section{Literature Review}




\subsection{Diabetes Mellitus}

Diabetes mellitus is a metabolic disease characterized by high blood glucose levels and insulin deficiency. There are different types of diabetes mellitus, all of which result in a hyperglycemic environment; however, they manifest differently. Type 1 and type 2 are the more common forms of diabetes mellitus. A third form, called gestational diabetes, occurs in pregnant women who have high blood glucose levels during pregnancy (4). Other types of diabetes mellitus due to other causes also exists, such as genetic defects in $\beta$-cell function and insulin action, different diseases or a drug and/or chemical induction of the disease (5). Previously, the diagnosis of diabetes mellitus was based on fasting plasma glucose or an oral glucose tolerance test; however, in 2010, the American Diabetes Association included the assessment of glycated hemoglobin $(\mathrm{HbA1c})$ into the criteria used to diagnose an individual with diabetes mellitus (5). Table 1.1 summarizes the specific criteria used to diagnose diabetes mellitus.

Table 1.1. American Diabetes Association: Criteria for the diagnosis of diabetes

\begin{tabular}{|l|l|}
\hline HbA1c & $\begin{array}{l}\geq 6.5 \% \text { (Using a NGSP certified method and standardized } \\
\text { to the DCCT reference assay) }\end{array}$ \\
\hline Fasting Plasma Glucose & $\geq 126 \mathrm{mg} / \mathrm{dL}$ (No caloric intake for $\geq 8$ hours) \\
\hline 2-hour Plasma Glucose & $\begin{array}{l}\geq 126 \mathrm{mg} / \mathrm{dL} \text { during an OGT test (Glucose load }=75 \mathrm{~g} \\
\text { anhydrous glucose in dissolved water) }\end{array}$ \\
\hline Random Plasma Glucose & $\geq 200 \mathrm{mg} / \mathrm{dL}$ in patients with symptoms of hyperglycemia \\
\hline $\begin{array}{l}\text { Note: This table is from the American Diabetes Association (5). NGSP = National Glycohemoglobin } \\
\text { Standardization Program; DCCT = Diabetes Control and Complications Trial; and OGT = Oral Glucose } \\
\text { Tolerance }\end{array}$
\end{tabular}




\subsubsection{Type 1 Diabetes Mellitus}

Type 1 diabetes mellitus makes up about $5-10 \%$ of all cases and is characterized by the lack of insulin production (136). Previously known as juvenile diabetes, insulindependent diabetes or type 1 diabetes mellitus is caused by pancreatic $\beta$-cell autoimmune destruction and subsequently, results in the inability for insulin production causing an insulin deficiency. This depletion in insulin levels results in the inability for the body to maintain glucose homeostasis, producing the hyperglycemic environment that is associated with diabetes mellitus. The rate of $\beta$-cell destruction is variable, with the onset occurring quickly in infants and children, to slowly in adults (12). Some forms of type 1 diabetes mellitus have no origination and are inherited, with these particular patients having insulinopenia and an increased susceptibility to ketoacidosis (12).

Type 1 diabetes is known to affect a multitude of organs and systems, including eyes, liver, kidney, nervous system, skeletal muscle, and the heart (45). The main treatment for type 1 diabetes mellitus is glucose monitoring and insulin therapy, which will aid in the utilization of glucose. Although type 1 diabetic patients are not normally obese, diet and exercise allow the body to accept the insulin therapy more efficiently and consequently, is recommended. Other potential treatments include cell-based therapeutics that target immune cells in attempt to prevent against, or reverse, type 1 diabetes mellitus (14). 


\subsubsection{Type 2 Diabetes Mellitus}

The more prevalent form of diabetes mellitus is classified as type 2, making approximately $90-95 \%$ of cases. Also known as non-insulin dependent diabetes, Type 2 diabetes mellitus is characterized by insulin resistance and a $\beta$-cell secretory defect, also resulting in a hyperglycemic environment (136). Patients with type 2 diabetes mellitus are often obese; however, it is possible to be non-obese with this disease. These particular patients may have an increased body fat percentage, distributed mainly in the abdominal region (12). In contrast to type 1 diabetes mellitus, ketoacidosis is not usually present. Type 2 diabetes mellitus is more common in African, Native, and Asian Americans, as well as Latinos and the aged population (4).

Patients with type 2 diabetes mellitus frequently go undiagnosed because of the slow disruption of the glucose homeostasis. These patients may have normal insulin levels; however, when compared to the elevated levels of blood glucose, the insulin levels are not high enough to compensate for the elevated blood glucose resulting in insulin secretion deficiency. At the beginning stages of type 2 diabetes mellitus, patients exhibit dyslipidemia and a lipoprotein pattern including increased triglyceride levels, low high density lipoprotein cholesterol values and small, dense low density lipoprotein

particles (141). Dyslipidemia is a critical risk factor to target in attempt to prevent cardiovascular disease in patients with type 2 diabetes. 
The main treatment of type 2 diabetes is diet and exercise to reduce the body weight in obese patients and to improve glycemic control $(141,158)$. By lowering body weight, blood glucose levels decrease; and therefore, the amount of insulin the pancreas secretes would be sufficient to maintain glucose homeostasis. Insulin therapy or medications that increase insulin sensitivity and/or decreased glucose production can also serve as treatment for type 2 diabetes mellitus (120).

\subsubsection{Significance}

Patients with diabetes mellitus are at an increased risk for cardiovascular complications, which are the leading cause of morbidity and mortality in patients with diabetes mellitus (66). In $2011,8.3 \%$ of the population in the United States was diagnosed with diabetes mellitus. Every year, there are 1.9 million new cases diagnosed in individuals 20 years old or older $(4,34)$. By the year 2030, the incidence of diabetes mellitus is expected to reach 366 million (161). This vastly growing disease is quickly becoming an epidemic; therefore, the evaluation and identification of the mechanisms

underlying the deleterious effects of diabetes mellitus can advance the biomedical field, from which therapeutic strategies can be developed and implemented. 


\subsection{Diabetic Cardiomyopathy}

In 1999, Grundy et al. (70) suggested that the most common complication of diabetes is cardiovascular disease. In the presence of hypertension and ischemic heart disease, patients with diabetes mellitus have an increased risk to develop heart failure. This development of heart failure can also occur in asymptomatic patients, without the presence of the hypertension or heart disease, which is termed diabetic cardiomyopathy $(8,21,58,122,131)$. Characterized by cardiac contractile abnormalities and independent of vascular pathogenesis, diabetic cardiomyopathy was first described by Rubler et al. in 1972 (131) and has been associated with mitochondrial dysfunction (51, $70,122,136,147)$. Studies have also reported direct mitochondrial dysfunction associated with cardiac contractile dysfunction $(62,98)$ due to the decrease in ATP production (143).

\subsubsection{Cardiac contractile dysfunction}

The deleterious effects to the heart caused by the hyperglycemic environment associated with diabetes mellitus ultimately results in heart failure. Studies have reported increased heart failure rates in individuals with diabetes mellitus, even after correcting for covariables, such as age, hypertension, and coronary artery disease (11, 19, 109). Human studies have indicated increased left ventricular mass and systolic dysfunction $(51,65,144)$, which may contribute to the diabetic-associated decreased cardiac function (21). Experimental data collected from animal models indicate 
contractile dysfunction presence as early as one week post diabetic induction (134). Literature has also suggested that diastolic dysfunction precedes systolic dysfunction in diabetic cardiomyopathy $(18,60,100)$. In 2006 , Galderisi stated that diabetic patients often develop abnormal left ventricular relaxation while the systolic function is still normal (64).

Previously, cardiac catheterization was used to assess left ventricular diastolic function from which the rates of relaxation, filling and left ventricular wall stiffness was measured (105). More recently, non-invasive conventional echocardiography and tissue Doppler techniques have been used to identify and quantify diastolic dysfunction (57, 146, 171). In normotensive diabetic patients, left ventricular dysfunction was observed by increased pre-ejection period and a shorter left ventricular ejection time using conventional echocardiography (6). Impaired left ventricular relaxation and restrictive filling patterns have been identified using Doppler echocardiography in diabetic patients $(64,111)$. Carugo et al. observed increased left ventricular wall thickness and mass, as well as a reduced E/A ratio (mitral peak velocity of early $(E)$ and late $(A)$ ventricular filling) in normotensive diabetic patients (33). Dabkowski et al. reported decreased cardiac function as determined by decreased rates of contraction, relaxation and developed pressure in Langendorff - perfused diabetic mouse hearts (48). Studies examining diabetic rats have shown decreased cardiac contractile performance in perfused, working hearts $(87,151)$, including decreased systolic function as measured by decreased speed and prolongation of cardiac contraction $(59,118)$. Regan et al. 
demonstrated decreased ventricular compliance with no change is systolic function in diabetic dogs (121).

In the diabetic heart, increases in the polyol and hexosamine pathway flux, advance glycation end products, and superoxide production have been reported to contribute to the contractile dysfunction and are suggested to be induced by the hyperglycemic environment (26). Along with hyperglycemia, the reduced insulin action and hyperlipidemia has resulted in an increase in both oxidative stress and lipotoxicity, as well as an alteration in calcium homeostasis (136).

\subsubsection{Cardiac metabolism}

Diabetes mellitus influences cardiac metabolism due to an altered substrate supply and decreased insulin action (129). Normally, $70 \%$ of the ATP production in the heart is derived from fatty acid oxidation compared to $30 \%$ of the energy derived from glucose and lactate (8). In type 1 diabetic patients, glucose uptake in the heart is normal, suggesting that the limiting factor is insulin (8). In type 2 diabetics, myocardial GLUT4 is responsible for the translocation of glucose into the tissue in order for the heart to derive energy through glucose oxidation. During the shift of substrate utilization, GLUT4 content and translocation activity is decreased, reducing the rates of glucose oxidation (164). Along with a decrease in glucose transport into the tissue, glucose supply has also been reported to be decreased in isolated diabetic cardiomyocytes (40), as well as in diabetic patients (112). 
Hyperglycemia causes increased circulating free fatty acids and triglycerides, due to enhanced lipolysis and increased lipoprotein synthesis. Fatty acid transport across the plasma membrane does not require any hormone, such as insulin; therefore, intracellular fatty acids increase during diabetes mellitus, causing fatty acid oxidation, which becomes the heart's exclusive energy source (8). As diabetes develops, the increased FA substrates activate peroxisome proliferator-activated receptor alphamediated signaling, leading to the transcriptional induction of enzymes involved in beta oxidation (22). Peroxisome proliferator-activated receptor beta and AMP-activated protein kinase are also activated by diabetes mellitus, which promotes fatty acid utilization $(8,106)$ and turns on ATP generating processes, such as fatty acid oxidation and glycolysis $(75,81)$.

Fatty acid oxidation is known to inhibit glycolysis and glucose oxidation. Along with these inhibitions and decrease glucose uptake, calcium homeostasis is disrupted, contributing to diabetic cardiomyopathy. The calcium homeostasis can also be altered by decreased expression of ion transporters or the $\mathrm{Na}^{+} / \mathrm{Ca}^{+}$exchanger, which has been shown to take place during type $1(76,91)$ and type 2 diabetes $(17)$ in animal models.

\subsubsection{Oxidative stress}

Oxidative stress is reflected by the body's ability to balance between the manifestation and removal of reactive oxygen species (ROS). The hyperglycemic environment associated with diabetes mellitus, leads to an increase in ROS. Examples 
of ROS include superoxide, hydrogen peroxide and hydroxyl radicals. Research previously published in a streptozotocin (STZ) type 1 diabetic mouse model suggested an increased in superoxide production (48). The body defends against the increase in ROS through an antioxidant defense system, which contains antioxidant enzymes that either inhibit ROS from being formed or scavenges ROS in attempt to maintain homeostasis. The antioxidant levels in the heart are lower in the presence of hyperglycemia, rendering the heart more susceptible to ROS damage (129).

Literature has suggested that the polyol and hexosamine pathway flux, advance glycation end products, and activation of protein kinase C (26) are reported to be increased in the diabetic heart due to an increase in ROS production. Because much of the damage during diabetes mellitus is caused by increased ROS levels, strategies to decrease ROS and subsequent oxidative damage are suggested to have therapeutic potential (69). One study reported that different antioxidants administered to GotoKakizaki rats, a type 2 diabetic rodent model, did not reverse the diabetic phenotype (114). In contrast, mitochondrial phospholipid hydroperoxide glutathione peroxidase overexpression has been shown to improve cardiac and mitochondrial function during a type 1 diabetic insult in mouse hearts (15). Taken together, studies have identified an increase in ROS production during diabetes mellitus, and as a result, alleviating the increased oxidative environment could serve as a potential therapeutic treatment for diabetes mellitus. 


\subsection{Mouse models of diabetic cardiomyopathy}

The mechanisms underlying human diabetic cardiomyopathy are still only partly understood. In order to understand diabetic cardiomyopathy more completely, both type 1 and type 2 rodent models have been used. Even though both type 1 and type 2 models show increased fatty acid utilization, decreased glucose utilization, and mitochondrial dysfunction (29), differences also exist between the two phenotypes. For example, increased ROS production in the hearts of type 2 diabetic models have been determined $(28,77)$, as well as fatty acid-mediated mitochondrial uncoupling induced by an increase in fatty acids $(24,28)$. Regardless of the overall similarities and differences between the groups, each model has helped to advance our understanding of the progression of diabetic cardiomyopathy.

Much of the research conducted in this area has employed mouse models, due to their inability to develop atherosclerosis (unless modified to do so). Our laboratory utilizes the type 1 diabetic STZ mouse model. STZ is a glucosamine-nitrosourea antibiotic that is structurally similar to glucose and is taken into pancreatic $\beta$-cells by the GLUT2 receptor. Through an ROS-mediated mechanism, STZ damages the pancreatic $\beta$-cell from which insulin cannot then be produced (145). Studies use both high dose and low dose STZ to render models type 1 diabetic. Our laboratory follows the protocol of the Animal Models of Diabetic Complications Consortium using multiple low-dose (50 $\mathrm{mg} / \mathrm{kg}$ body weight STZ dissolved in sodium citrate buffer) STZ injected intraperitoneally over five consecutive days. This model was chosen because it is most widely used and 
does not induce deleterious effects associated with diabetes mellitus (165). Using this method, cardiac dysfunction has been observed by decreased rates of relaxation, contraction, developed pressure and systolic pressure $(48,90,155)$ compared to controls. The increased levels of ROS and superoxide production affect the antioxidant capacity in the mitochondria, rendering mitochondria dysfunctional in the STZ model $(48,67,140)$. Studies have identified an increase in fatty acid oxidation, along with lipid storage, while glucose oxidation is decreased in the STZ model $(37,61,84)$. Calcium handling is also decreased in the STZ model; consequently impacting cardiac function $(42,62,76)$.

Alloxan is another chemical used to induce type 1 diabetes mellitus. Alloxan produces superoxide radials that eventually form ROS, which disrupts pancreatic $\beta$-cells and subsequently prevents insulin production (145). One study using alloxan-induced type 1 diabetic mice reported insulin secretory impairment in response to glucose (7). Turko et al. (149) found a common nitrating species that may underlie the nitration involved in mitochondria dysfunction in alloxan-susceptible mice. Aasum et al. also observed decreased systolic and diastolic function (1).

Other type 1 diabetic models include the OVE26 mouse, the Akita mouse, the non-obese diabetic (NOD) mouse, and the BioBreeding (BB) rat. The OVE26 mouse was developed in 1989 by Epstein et al. (56) and was reported to have decreased cardiac contractility and mitochondrial function, along with increased oxidative stress $(137,138,167)$. The Akita mouse, discovered in 1997 by Dr. Akio Koizumi (168), was 
reported to have increased mitochondrial content, even though overall mitochondrial function was decreased (30, 31). First reported in 1980 (102) NOD mice have a susceptibility to spontaneously develop type 1 diabetes mellitus (93). This model appears to be used more for immunological responses on the development of diabetes $(55,95)$. The BB rat is a model of human type 1 diabetes mellitus, from which two inbred lines have been created: one that is prone to diabetes and the other is diabetic resistant (160). The diabetic prone BB develops type 1 diabetes mellitus within 2 to 3 months. The diabetic resistant needs to be virally infected in order to develop diabetes (88). Interestingly, Rodrigues and McNeill observed cardiac dysfunction in perfused hearts from diabetic-prone BB rats, similar to the dysfunction observed in STZ rats and the human population (126).

Rodent models of type 2 diabetes include the ob/ob mouse, the $\mathrm{db} / \mathrm{db}$ mouse, and the Zucker diabetic fatty rat. Both the ob/ob and $\mathrm{db} / \mathrm{db}$ mouse were derived by targeting the same mechanism. The ob/ob mouse resulted from a mutation in the leptin gene (172), where as the $\mathrm{db} / \mathrm{db}$ is a consequence of an impaired leptin receptor (44). The ob/ob mouse develops type 2 diabetes by 15 weeks, even though they display higher insulin levels and lower glucose tolerance by only 5 weeks (27). Compared to the ob/ob, the db/db mouse is has a normal glucose tolerance at 4 weeks, but develops type 2 diabetes at 8 weeks (27). Due to the earlier manifestation of type 2 diabetes, the $\mathrm{db} / \mathrm{db}$ mouse has greater contractile abnormalities (170). In both models, fatty acid oxidation rates and oxidative stress levels are increased, while glucose oxidation rates 
are decreased $(17,24,27,29,99,103,170)$. Mitochondrial dysfunction is also evident in both models $(23,24)$.

The Zucker diabetic fatty rat, like the $\mathrm{db} / \mathrm{db}$, contains a mutation in the leptin receptor, which is the cause of type 2 diabetes mellitus. Hyperglycemia develops around 6 weeks of age; however, the high blood glucose levels are not stable until 10 12 weeks, when the insulin levels start to fall (29). The Zucker diabetic fatty rat has not been studied as extensively as the mouse type 2 diabetic models. Even though cardiac size is increased in this model (29), cardiac function is decreased $(117,169)$. The decreased contractile function could be contributed to the increase in fatty acid availability, which would also increase fatty acid oxidation (169). Carbohydrate oxidation is also seen to decrease during the type 2 diabetic state (38).

\subsection{Mitochondrial Subpopulations}

Two distinct mitochondrial subpopulations are present in striated muscle: subsarcolemmal (SSM) and interfibrillar mitochondria (IFM) $(48,116,124)$ (Figure 1.1). Each population is spatially, morphologically and functionally distinct. The SSM are located just beneath the sarcolemma and produce primarily operate to actively transport electrolytes and metabolites across the sarcolemma. They are larger in size and contain lamelliform cristae (124). The IFM are situated between the myofibrils, are smaller, more compact in size. Their cristae are lamelliform and tubular in shape, allowing for a 
higher concentration of protons, and subsequently, higher ATP synthase activity (124). Compared to the SSM, the IFM have higher membrane potential and state 3 respiration rates, as well as increased ATP production $(157,163)$. Their main function is suggested to be to generate ATP for muscle contraction (48).

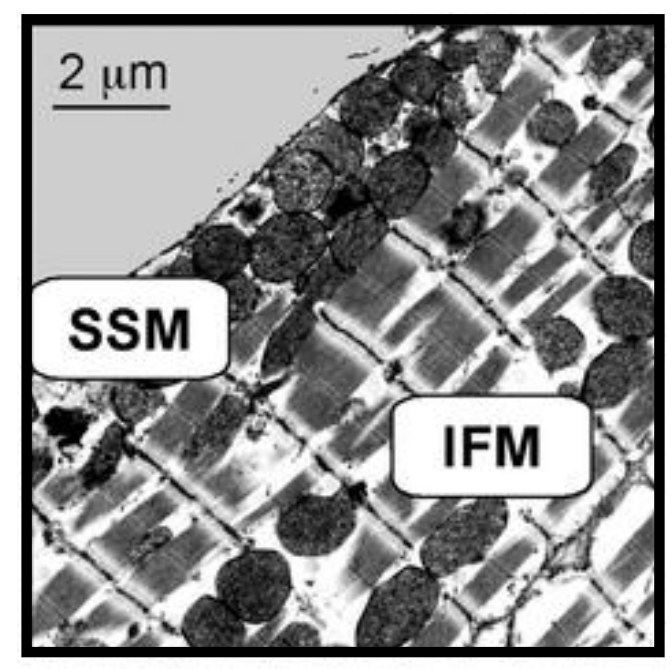

Figure 1.1. Mitochondrial subpopulations. Electron micrograph of the placement of subsarcolemmal mitochondria (SSM) and interfibrillar mitochondria (IFM) in the heart muscle (in situ) (79).

Literature has suggested that the two mitochondrial pools respond differently to pathological insults, such as ischemia/reperfusion, aging, heart failure and diabetes (39, $47,48,80,97,123,130)$. Chen et al. reported decreased mitochondrial respiration through cytochrome oxidase, as well as increased hydrogen peroxide production from electron transport chain (ETC) complexes I and III in both SSM and IFM after an ischemic insult (39). Although, Lesnefsky et al. also reported an ischemia-mediated mitochondrial respiration decrease through cytochrome oxidase, the SSM were the only subpopulation affected (97). Aging also greatly impacts mitochondrial function. In 2002, Hoppel et al. (80) reported decreased oxidative phosphorylation through ETC complex III and IV, specifically in the IFM, of aged rats compared to control (80). Rosca et al. 
reported increased ETC complex I and complex III in the SSM, with no change in ETC complex IV in canine coronary heart failure model as measured by Native PAGE (130). Type 1 and type 2 diabetes mellitus affect the mitochondrial subpopulations. Interestingly, type 1 diabetes mellitus has been shown to affect the IFM (48), whereas type 2 diabetes affects the $\operatorname{SSM}(47,123)$. Although the presence of hyperglycemia leads to the phenotype of both type 1 and type 2 diabetes mellitus, it is unclear as to why mitochondrial subpopulations are differentially affected. It has been suggested that the spatial arrangement and environment of the distinct subpopulations allow for the mitochondria to be impacted differently in response to pathological insults. For example, type 2 diabetes mellitus is characterized by high levels of triglyceride accumulation and lipid deposition (110), which could be the potential mechanism contributing to the dysfunctional SSM, when compared to IFM during a type 2 diabetic insult, because of the sub-sarcolemmal location of the SSM (110).

\subsection{Mitochondrial dysfunction in the diabetic heart}

Hyperglycemia is a recognized complication of both type 1 and type 2 diabetes (132) and in combination with oxidative stress and subsequent increased production of ROS, deleterious effects cause the heart and the organelles that reside within, such as mitochondria, to become dysfunctional $(125,128,138)$. Mitochondria are the main source of ROS in cells and the effects of ROS damage have been linked to different pathologies, such as ischemia/reperfusion injury, atherosclerosis, and diabetes mellitus 
$(52,115)$. Literature suggests that the increase in ROS production is the unifying mechanism underlying mitochondrial dysfunction during both type 1 and type 2 diabetes mellitus (26).

\subsubsection{Mitochondrial oxidative stress}

Mitochondria are more vulnerable to oxidative stress than other organelle because the inner mitochondrial membrane (156) is the primary source of ROS production, specifically at ETC complex I and complex III (115). ROS are suggested to induce lipid modifications, DNA damage and protein damage (153). Along with the elevated levels of free fatty acids observed during diabetes mellitus, lipid peroxidation and membrane disruption are more likely to occur $(53,68)$ given the increase in ROS production. Our laboratory reported increased damage to proteins and lipids, as determined by nitrotyrosine content and lipid peroxidation in type 1 diabetic IFM, respectively (48). Interestingly, animal models manipulated to overexpress players involved in the antioxidant defense system, such as manganese superoxide dismutase and mitochondrial phospholipid hydroperoxide glutathione peroxidase, have provided protection against mitochondrial dysfunction associated with diabetes mellitus $(15,137)$.

\subsubsection{Calcium homeostasis}

The uptake of calcium into mitochondria regulates mitochondrial metabolism (54) and acts to stimulate oxidative phosphorylation $(13,49,74,104)$. When needed, the 
calcium influx is increased enhancing ATP production in order to meet the ATP demand needed to increase contractile force. In the presence of a pathological stimulus, calcium entering mitochondria increases ROS by increasing oxidative phosphorylation. Alteration in calcium homeostasis can also activate the opening of the mitochondrial permeability transition pore (mPTP) and subsequently trigger apoptosis (25). One study using type 1 diabetic rats, reported decreased calcium uptake, along with increased mPTP opening (113). In agreement, our laboratory observed increased mPTP opening in the IFM of type 1 diabetic mouse hearts (162). In conjunction with the increased mPTP opening, an observed increase in cytochrome $c$ release suggested damage to the anchoring phospholipid environment, also an effect of increased ROS generation.

\subsubsection{Lipotoxicity}

Obesity associated with diabetes mellitus is linked to excess lipid deposition in multiple tissues, including the heart (159). The tissues can store some of the lipid deposition as triglycerides; however, the excess lipid deposition can cause lipotoxic effects, affecting multiple processes. For example, the excess lipids are forced through the oxidative machinery, causing mitochondrial dysfunction (166) and cardiac dysfunction (78).

In regards to mitochondrial dysfunction, augmented lipid accumulation increases mitochondrial fatty acid oxidation, which in turn alters different pathways that all lead to cardiac contractile dysfunction. Literature has reported reduced ATP generation in the 
presence of increased fatty acid oxidation indicative of mitochondrial uncoupling (23). Further, an increase in fatty acid oxidation is associated with increased ROS production (154), which then oxidizes lipids contributing to mitochondrial and cardiac dysfunction.

\subsubsection{Mitochondrial dysfunction in the type 1 diabetic heart}

Even though mitochondrial dysfunction in the type 1 diabetic heart has been identified, the mechanisms underlying the dysfunction are still being evaluated. Mitochondrial respiration has been reported to be decreased during type 1 diabetes mellitus using different substrates $(48,96,152)$. Proteomic alterations have been identified in the type 1 diabetic heart, with IFM being affected to a greater extent than

SSM (16). We and others have shown a decrease in ETC function during a type 1 diabetic insult, specifically in IFM $(48,96)$. ROS production has been shown to be increased in type 1 OVE26 mice (142), as well as an increase in apoptotic susceptibility, specifically in the IFM (162), all of which contributing to the observed mitochondrial dysfunction.

\subsubsection{Mitochondrial dysfunction in the type 2 diabetic heart}

Many of the same characteristics of mitochondrial dysfunction reported from type 1 animal models are also reported from type 2 animal models; however, the diabetic phenotypes between the two types include important differences. It is interesting to point out that much of the mitochondrial dysfunction occurs in the SSM to a greater 
extent than the IFM during a type 2 diabetic insult, which is in contrast to type 1 diabetes mellitus. Obesity is associated with type 2 diabetes mellitus; therefore, increases in lipid deposition cause much of the dysfunction during type 2 diabetes. Mitochondrial respiration was reported to be decreased in the presence of both glutamate and palmitoylcarnitine in a type 2 diabetic animal model, specifically in the SSM (47). This finding is supported by total mitochondria isolated from human atrial tissues, from which mitochondrial respiration in the presence of palmitoylcarnitine is also decreased (9). The increased lipid deposition leads to increased lipid peroxidation and subsequent ROS production. Further contributing to mitochondrial dysfunction associated with type 2 diabetes mellitus is the proteomic alterations observed in the diabetic SSM, of which many are involved in mitochondrial processes that are housed in the IMM (156).

\subsection{Inner Mitochondrial Membrane}

A critical component of the mitochondrion is the IMM (156) (Figure 1.2), which is composed of a specific lipid environment that houses many proteins required for proper mitochondrial function. This membrane is the primary source of ROS generation, making the IMM susceptible to oxidative damage. Subsequently, the integrity of the lipid environment of the IMM is altered due to the increase in ROS and oxidative damage, further enhancing mitochondrial dysfunction (159). The increased ROS target the lipids

of the IMM due to their highly unsaturated structure, causing enhanced lipid 
peroxidation (85) leading to disassociation of lipids and critical proteins that reside in the IMM, such as the ETC complexes (2). During type 1 diabetes mellitus, ETC proteins are decreased (16) along with their associated activities (48).

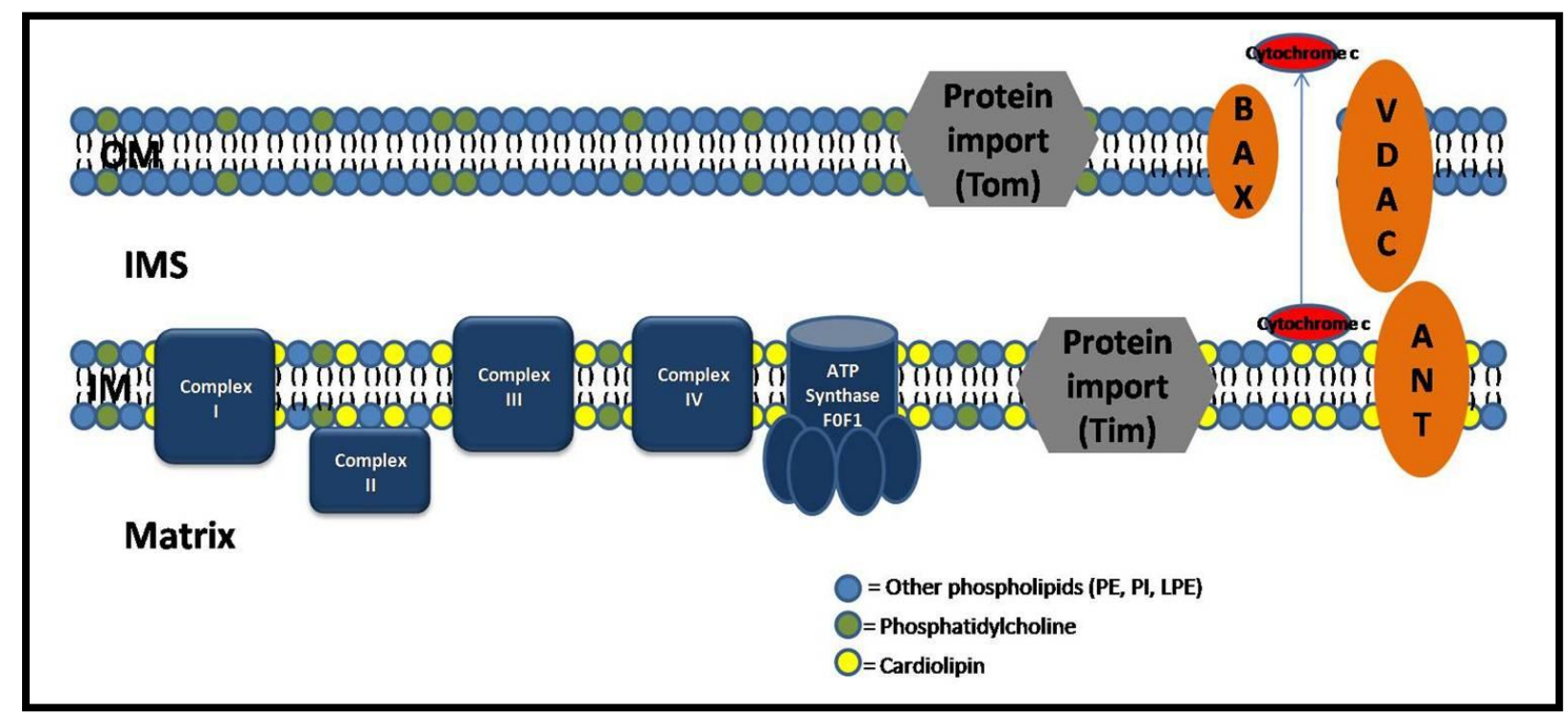

Figure 1.2. Schematic of the inner mitochondrial membrane. $P E=$ Phosphatidylethanolamine; PI = Phosphatidylinositol; LPE = Lyso-phosphatidylethanolamine; VDAC $=$ voltage dependent anion channel; and ANT = adenine nucleotide transporter .

\subsubsection{Inner mitochondrial membrane machinery}

The IMM houses many proteins required for proper mitochondrial function, including the machinery that produces ATP, ETC complexes, and protein import machinery (Figure 1.2). Proteomic data has revealed that the IMM contains the highest percentage of proteins affected by a diabetic insult (16). During a type 1 insult, IFM proteins are affected to a greater extent compared to SSM proteins. Many of these altered proteins are also affected during a type 2 diabetic insult, with the SSM being 
affected to a greater extent (47). An example of this phenomenon would be glucose regulated protein 75 (Grp75), which is a key constituent in the protein import process.

Also housed in the IMM are the constituents involved in mitochondrial-mediated apoptosis, such as the cytochrome $c$ anchoring mechanism and adenine nucleotide transporter, which forms a pore with voltage dependent anion channel to release cytochrome $c$. The release of cytochrome $c$ occurs when the anchoring lipids are damaged during a pathological insult, in this case, diabetes mellitus. The phospholipid environment is critical for the associated proteins to function properly. The impaired mitochondrial function reported during a diabetic insult can be partially explained by examining the IMM phospholipid environment, which contains a unique phospholipid, cardiolipin.

\subsubsection{Cardiolipin and the Biosynthesis Pathway}

Cardiolipin is thought to play a key role in mitochondrial structure and bioenergetics (2). Most abundant in the mammalian heart, cardiolipin is primarily located in the IMM (133); however, literature suggests the presence of cardiolipin in the outer mitochondrial membrane $(50,83)$. Cardiolipin is a unique phospholipid with a dimeric structure containing three glycerol backbones and four fatty acyl side chains (133) (Figure 1.3). 


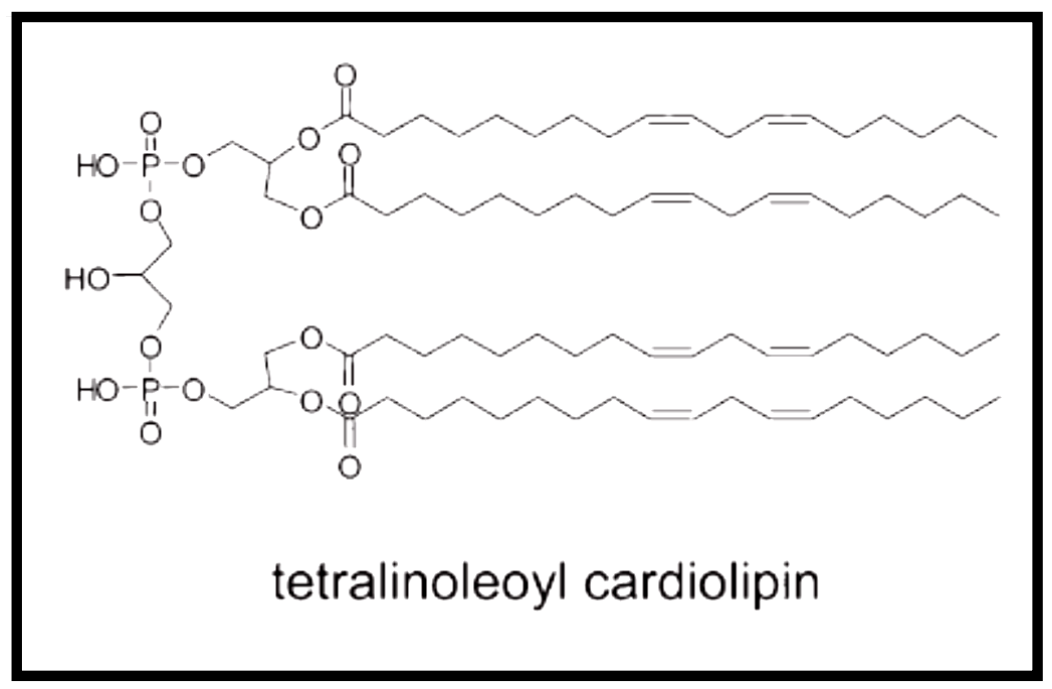

Figure 1.3. Structure of tetralinoleoyl cardiolipin. Adapted from (94)

The biosynthetic pathway of cardiolipin starts in the outer mitochondrial membrane where phosphatidic acid is converted into cytidine diphosphate diacylglycerol (CDP-DAG) by cytidine diphosphate diacylglycerol synthase (CDS). Phosphatidylglycerol synthase (PGS) then converts CDP-DAG to phosphatidyl-glycerol phosphate (PG-P), where the phosphate is removed by phosphatidyl-glycerol phosphatase (PGP), resulting in the formation of phosphatidylglycerol (PG). Cardiolipin synthase (CRLS) catalyzes the condensation of CDP-DAG with PG, resulting in a nascent form of cardiolipin $(41,101)$. Because the substrates used to make cardiolipin do not contain the precise fatty acyl side chains for the specific tissue, a remodeling process takes place converting the nascent form of cardiolipin to the mature form containing specific fatty acyl side chains (41) (Figure 1.4). 


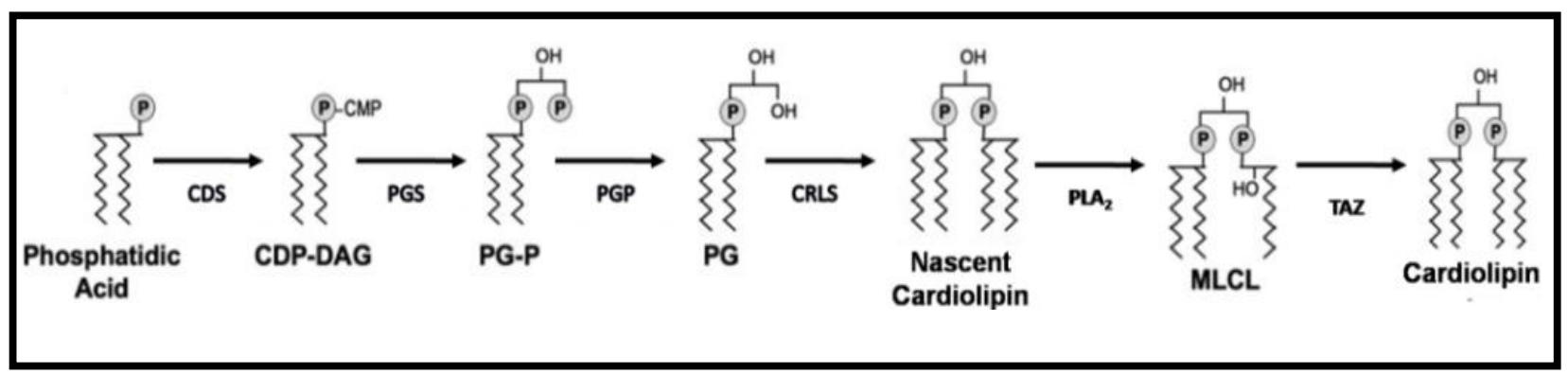

Figure 1.4. Cardiolipin biosynthetic pathway. Schematic illustrating cardiolipin synthesis. $P L A_{2}=$ phospholipase $A_{2} ; M L C L=$ monolyso-cardiolipin; $T A Z=$ Tafazzin. Refer to text for other abbreviations.

Depending on the tissue and environment, the side chains of cardiolipin are composed of different fatty acids. Linoleic acid is the most abundant fatty acid side chain found to compose the mature form of cardiolipin in cardiac mitochondria, constituting about $80 \%$ of all cardiolipin $(3,133)$ (Figure 1.3). Each side chain of a tetralinoleoyl cardiolipin contains eighteen carbons with two double bonds, with the double bonds serving as targets for ROS and oxidative damage $(82,85)$. The side chains of cardiolipin create a conical shaped lipid (82), allowing it to interact with IMM proteins and facilitate proper mitochondrial function.

Cardiolipin has been suggested to be involved in protein import $(82,148)$, membrane stability (133), and cytochrome $c$ attachment $(85,119)$. Other proteins that rely on cardiolipin for proper function and activity are carriers of the IMM, such as adenine nucleotide translocator (20), and carnitine (86), pyruvate and phosphate carriers $(89,107)$. Literature suggests an interaction between cardiolipin and the ETC 
complexes involved in oxidative phosphorylation (133), such as ETC complex I, III, IV, and ATP synthase $(63,135)$.

This important lipid has been shown to play a critical role in the organization of mammalian mitochondrial ATP synthase. It has been suggested that the IMM-bound $\mathrm{F}_{0}$ complex of ATP synthase contains high affinity binding sites for cardiolipin (2); however, these specific binding sites are unknown. Cardiolipin is also known to trap protons within its polar head group, serving as a proton reservoir to maintain IMM potential and to contribute to ATP synthesis (72). Shotgun lipidomics have revealed decreased tetralinoleic cardiolipin content in diabetic mouse hearts and the authors have suggested a possible mechanistic reliance on the interaction between IMM protein activity and cardiolipin (73).

\subsection{Protein Import}

There are over 1500 proteins in the human mitochondria, of which only 13 are transcribed and translated in the organelle $(10,32)$. The remaining $99 \%$ of the nuclear encoded mitochondrial proteins need to be imported into the mitochondria through a complex process. Proteomic analyses indicate a decreased abundance of nuclear encoded proteins during a diabetic insult, which included proteins essential for proper mitochondrial import processes $(46,150)$. 


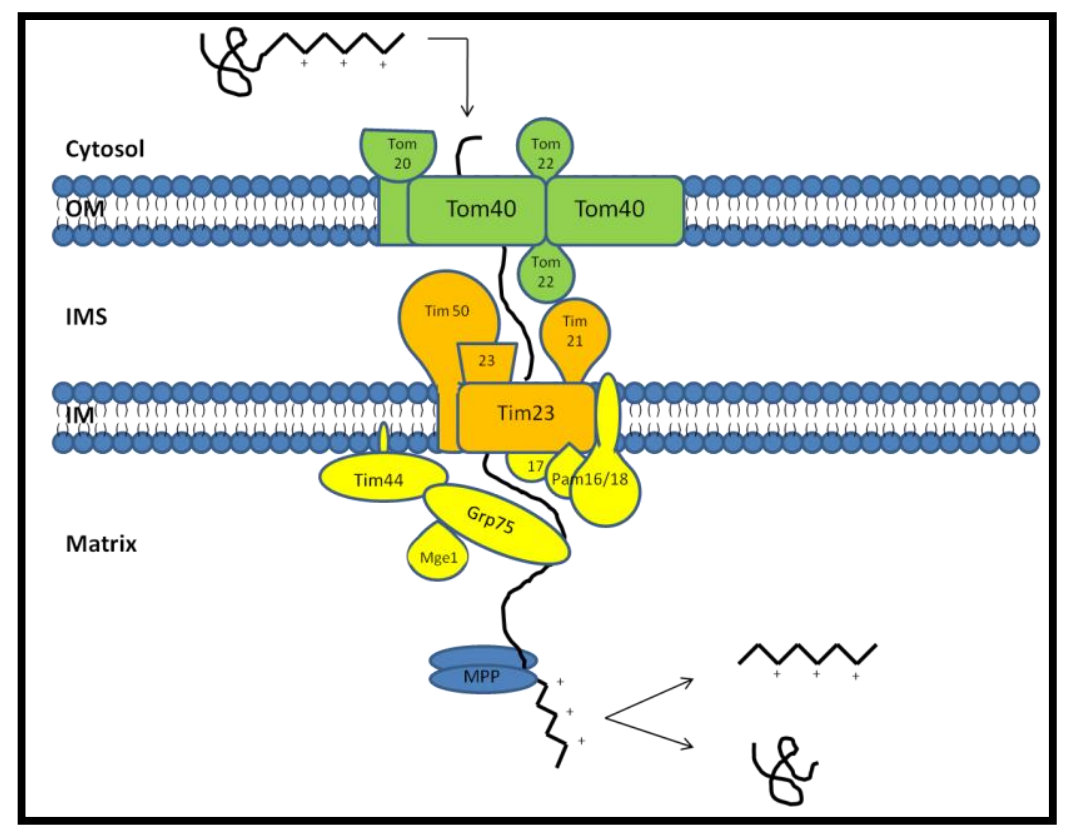

Figure 1.5. Mitochondrial import of matrix-targeted proteins. Schematic illustrating the protein import process (92).

Protein import is mutli-step, complex pathway from which nuclear-encoded proteins enter the mitochondria (Figure 1.5). The constituents involved in this process, outer membrane translocases (Tom), inner membrane translocases (Tim), and the presquence translocase-associated motor (PAM), create a supercomplex that facilitates the movement of proteins into the matrix (36). The protein import process begins outside of the mitochondrion, where $\mathrm{N}$-terminal pre-proteins are recognized by outer mitochondrial membrane receptors Tom20 and Tom22. The pre-proteins then pass through Tom40 and enter the inner mitochondrial space. Proteins that are targeted for the matrix will then bind to the main IMM protein import pore, Tim23, with the help of Tim21 and Tim50. Tim50 recruits the PAM complex, which pulls the pre-protein into the matrix. The key constituent of the PAM complex, Grp75, also known as mortalin or 
mitochondrial heat shock protein 70 (mthsp70), binds to Tim44 on the matrix side and through an energy-driven mechanism, diassociates from Tim44, pulling the pre-protein completely into the matrix (156). Matrix-processing peptidase (MPP) cleaves the targeting sequence from the pre-protein. The protein is then properly hsp60 and hsp10, resulting in a mature protein in the mitochondrial matrix $(35,71,92,108,139)$. Depending on the $\mathrm{N}$-terminal presequence, the protein can be targeted for either the matrix, IMM, outer mitochonrial membrane or inter mitochondrial membrane space.

Mitochondrial protein import has been shown to be decreased in a type 1 diabetic setting, specifically in the IFM (15). By using a novel mitochondrial protein import assay, protein import rates into mitochodria can be measured (15). Figure 1.6A is a vector map that illustrates the MitoGFP plasmid that is grown in a cell-free bacterial protein lysate system. When the lysate is incubated with mitochondria in the presence of substrates necessary for the import process to occur, protein import of MitoGFP1 is initiated. Following a 10 minute incubation period, import is stopped and after processing the mitochondria, a Western blot is performed to determine the amount of MitoGFP1 that has been imported into the mitochondria. Figure 1.6B illustrates the resulting Western blot probed for MitoGFP1, with lane 1 representing mitochondria without the lysate, lane 2 depicting the lysate without mitochondria, whereas lane 3 demonstrates the presence of MitoGFP1 when mitochondria and the lysate are incubated together. The top band, measured at $31 \mathrm{kDa}$, respresents the unprocessed mitoGFP1 that has not yet been imported into the mitochondria. The bottom band, 
measured at $28 \mathrm{kDa}$, respresents the processed mitoGFP1that has been imported into the mitochondria.

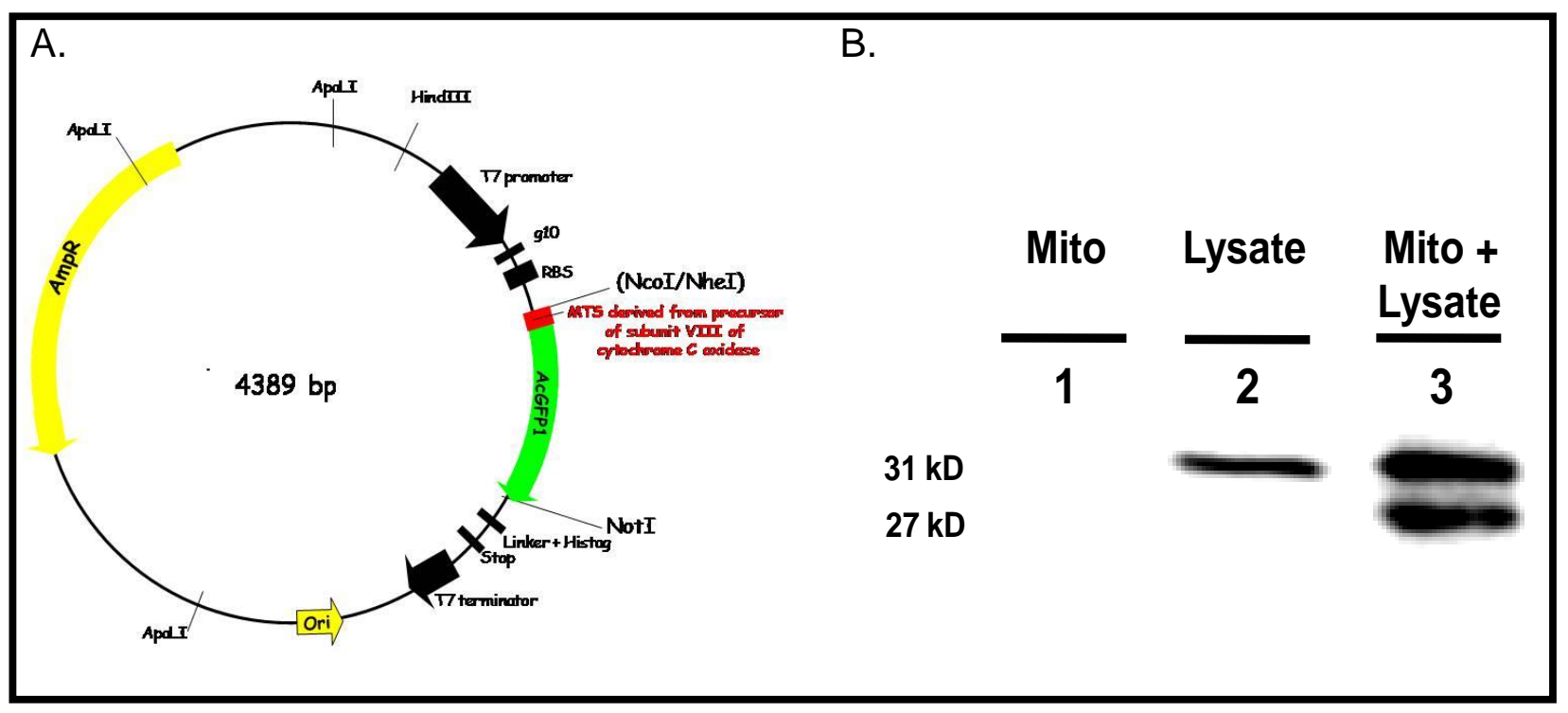

Figure 1.6. MitoGFP1 construct and protein import. (A.) pMITOGFP1 plasmid. (B.) Western blot of MitoGFP1 protein import into isolated cardiac mitochondria probed for MitoGFP1 in samples containing mitochondria only (lane 1), MitoGFP1 protein lysate (lane 2), or combined mitochondria and lysate (lane 3). Upper $31 \mathrm{kD}$ band represents the full length precursor MitoGFP1 un- processed; lower $27 \mathrm{kD}$ band represents mature cleaved MitoGFP1 protein residing within the mitochondrial matrix.

Baseler et al. previously reported protein import deficiency in type 1 diabetic mice, specifically in the IFM (16). The deficiency was attributed to decreased Grp75 protein content in the diabetic IFM compared to control, with no observed differences in 
the SSM regarding protein import or Grp75 protein content (16). To date, protein import has not been reported in a type 2 diabetic mouse model, nor in a diabetic human model.

\subsection{Summary}

In the United States, heart failure is the leading cause of mortality in patients with DM (66). In 2010, 11.3\% of the U.S. population age 20 and older suffered from diabetes (43) and each year, approximately 15,000 more people over the age of 20 are diagnosed with type 1 diabetes (127). Cardiovascular complications that arise from diabetes mellitus, such as diabetic cardiomyopathy, are becoming a more prevalent tool used for the diagnosis of heart failure; however, possible mechanisms involving mitochondrial dysfunction associated with diabetic cardiomyopathy need to be further examined to advance our understanding of the disease and its development. The goal of this dissertation was to evaluate the impact of type 1 diabetes mellitus on the IMM phospholipid environment, specifically cardiolipin, and the processes that are housed within. Further, the mitochondrial dysfunction in the type 2 diabetic human heart was examined with the intention of discovering potential targets for future therapeutic strategies. 


\subsection{References}

1. Aasum E, Belke DD, Severson DL, Riemersma RA, Cooper M, Andreassen M, and Larsen TS. Cardiac function and metabolism in Type 2 diabetic mice after treatment with BM 17.0744, a novel PPAR-alpha activator. Am J Physiol Heart Circ Physiol 283: H949-957, 2002.

2. Acehan D, Malhotra A, Xu Y, Ren M, Stokes DL, and Schlame M. Cardiolipin affects the supramolecular organization of ATP synthase in mitochondria. Biophys $J$ 100: 2184-2192, 2011.

3. Acehan D, Vaz F, Houtkooper RH, James J, Moore V, Tokunaga C, Kulik W, Wansapura J, Toth MJ, Strauss A, and Khuchua Z. Cardiac and skeletal muscle defects in a mouse model of human Barth syndrome. J Biol Chem 286: 899-908, 2011.

4. ADA. American Diabetes Association: Diabetes Basics, 2013.

5. ADA. Standards of Medical Care in Diabetes -2013. Diabetes Care 36: S11-S66, 2013.

6. Ahmed SS, Jaferi GA, Narang RM, and Regan TJ. Preclinical abnormality of left ventricular function in diabetes mellitus. Am Heart J 89: 153-158, 1975.

7. Ahren B and Sundkvist G. Long-term effects of alloxan in mice. Int J Pancreatol 17: 197-201, 1995.

8. An D and Rodrigues B. Role of changes in cardiac metabolism in development of diabetic cardiomyopathy. American Journal of Physiology - Heart and Circulatory Physiology 291: H1489-H1506, 2006.

9. Anderson EJ, Kypson AP, Rodriguez E, Anderson CA, Lehr EJ, and Neufer PD. Substrate-Specific Derangements in Mitochondrial Metabolism and Redox Balance 
in the Atrium of the Type 2 Diabetic Human Heart. Journal of the American College of Cardiology 54: 1891-1898, 2009.

10. Anderson S, Bankier AT, Barrell BG, de Bruijn MH, Coulson AR, Drouin J, Eperon IC, Nierlich DP, Roe BA, Sanger F, Schreier PH, Smith AJ, Staden R, and Young IG. Sequence and organization of the human mitochondrial genome. Nature 290: 457-465, 1981.

11. Aronow WS and Ahn C. Incidence of heart failure in 2,737 older persons with and without diabetes mellitus. Chest 115: 867-868, 1999.

12. Association AD. Diagnosis and Classification of Diabetes Mellitus. Diabetes Care 33: S62-S69, 2010.

13. Balaban RS. Cardiac Energy Metabolism Homeostasis: Role of Cytosolic Calcium. Journal of Molecular and Cellular Cardiology 34: 1259-1271, 2002.

14. Barcala Tabarrozzi AE, Castro CN, Dewey RA, Sogayar MC, Labriola L, and Perone MJ. Cell-based interventions to halt autoimmunity in type 1 diabetes mellitus. Clinical \& Experimental Immunology 171: 135-146, 2013.

15. Baseler WA, Dabkowski ER, Jagannathan R, Thapa D, Nichols CE, Shepherd DL, Croston TL, Powell M, Razunguzwa TT, Lewis SE, Schnell DM, and Hollander JM. Reversal of mitochondrial proteomic loss in Type 1 diabetic heart with overexpression of phospholipid hydroperoxide glutathione peroxidase. Am J Physiol Regul Integr Comp Physiol 304: R553-565, 2013.

16. Baseler WA, Dabkowski ER, Williamson CL, Croston TL, Thapa D, Powell MJ, Razunguzwa TT, and Hollander JM. Proteomic alterations of distinct 
mitochondrial subpopulations in the type 1 diabetic heart: contribution of protein import dysfunction. Am J Physiol Regul Integr Comp Physiol 300: R186-200, 2011.

17. Belke DD, Larsen TS, Gibbs EM, and Severson DL. Altered metabolism causes cardiac dysfunction in perfused hearts from diabetic (db/db) mice. Am J Physiol Endocrinol Metab 279: E1104-1113, 2000.

18. Bell DSH. Diabetic Cardiomyopathy. Diabetes Care 26: 2949-2951, 2003.

19. Bertoni AG, Tsai A, Kasper EK, and Brancati FL. Diabetes and idiopathic cardiomyopathy: a nationwide case-control study. Diabetes Care 26: 2791-2795, 2003.

20. Beyer $\mathbf{K}$ and Klingenberg $\mathbf{M}$. ADP/ATP carrier protein from beef heart mitochondria has high amounts of tightly bound cardiolipin, as revealed by 31P nuclear magnetic resonance. Biochemistry 24: 3821-3826, 1985.

21. Boudina S and Abel ED. Diabetic cardiomyopathy revisited. Circulation 115: 3213-3223, 2007.

22. Boudina S and Abel ED. Diabetic cardiomyopathy, causes and effects. Rev Endocr Metab Disord 11: 31-39, 2010.

23. Boudina S, Sena S, O'Neill BT, Tathireddy P, Young ME, and Abel ED. Reduced mitochondrial oxidative capacity and increased mitochondrial uncoupling impair myocardial energetics in obesity. Circulation 112: 2686-2695, 2005.

24. Boudina S, Sena S, Theobald H, Sheng X, Wright JJ, Hu XX, Aziz S, Johnson JI, Bugger H, Zaha VG, and Abel ED. Mitochondrial energetics in the heart in obesity-related diabetes: direct evidence for increased uncoupled respiration and activation of uncoupling proteins. Diabetes 56: 2457-2466, 2007. 
25. Brookes PS, Yoon Y, Robotham JL, Anders MW, and Sheu S-S. Calcium, ATP, and ROS: a mitochondrial love-hate triangle. American Journal of Physiology Cell Physiology 287: C817-C833, 2004.

26. Brownlee M. The pathobiology of diabetic complications: a unifying mechanism. Diabetes 54: 1615-1625, 2005.

27. Buchanan J, Mazumder PK, Hu P, Chakrabarti G, Roberts MW, Yun UJ, Cooksey RC, Litwin SE, and Abel ED. Reduced cardiac efficiency and altered substrate metabolism precedes the onset of hyperglycemia and contractile dysfunction in two mouse models of insulin resistance and obesity. Endocrinology 146: 5341-5349, 2005.

28. Bugger H and Abel ED. Molecular mechanisms for myocardial mitochondrial dysfunction in the metabolic syndrome. Clin Sci (Lond) 114: 195-210, 2008.

29. Bugger H and Abel ED. Rodent models of diabetic cardiomyopathy. Dis Model Mech 2: 454-466, 2009.

30. Bugger H, Boudina S, Hu XX, Tuinei J, Zaha VG, Theobald HA, Yun UJ, McQueen AP, Wayment B, Litwin SE, and Abel ED. Type 1 diabetic akita mouse hearts are insulin sensitive but manifest structurally abnormal mitochondria that remain coupled despite increased uncoupling protein 3. Diabetes 57: 2924-2932, 2008.

31. Bugger H, Chen D, Riehle C, Soto J, Theobald HA, Hu XX, Ganesan B, Weimer BC, and Abel ED. Tissue-specific remodeling of the mitochondrial proteome in type 1 diabetic akita mice. Diabetes 58: 1986-1997, 2009. 
32. Calvo S, Jain M, Xie X, Sheth SA, Chang B, Goldberger OA, Spinazzola A, Zeviani M, Carr SA, and Mootha VK. Systematic identification of human mitochondrial disease genes through integrative genomics. Nat Genet 38: 576-582, 2006.

\section{Carugo S, Giannattasio C, Calchera I, Paleari F, Gorgoglione MG, Grappiolo}

A, Gamba P, Rovaris G, Failla M, and Mancia G. Progression of functional and structural cardiac alterations in young normotensive uncomplicated patients with type 1 diabetes mellitus. J Hypertens 19: 1675-1680, 2001.

34. CDC. National diabetes fact sheet: national estimates and general information on diabetes and prediabetes in the United States, 2011. : Atlanta, GA: U.S. Department of Health and Human Services, Centers for Disease Control and Prevention, 2011.

35. Chacinska A, Lind M, Frazier AE, Dudek J, Meisinger C, Geissler A, Sickmann A, Meyer HE, Truscott KN, Guiard B, Pfanner N, and Rehling P. Mitochondrial presequence translocase: switching between TOM tethering and motor recruitment involves Tim21 and Tim17. Cell 120: 817-829, 2005.

36. Chacinska A, Rehling P, Guiard B, Frazier AE, Schulze-Specking A, Pfanner N, Voos W, and Meisinger C. Mitochondrial translocation contact sites: separation of dynamic and stabilizing elements in formation of a TOM-TIM-preprotein supercomplex. Embo J 22: 5370-5381, 2003.

37. Chatham JC and Forder JR. Relationship between cardiac function and substrate oxidation in hearts of diabetic rats. Am J Physiol 273: H52-58, 1997.

38. Chatham JC and Seymour AM. Cardiac carbohydrate metabolism in Zucker diabetic fatty rats. Cardiovasc Res 55: 104-112, 2002. 
39. Chen Q, Moghaddas S, Hoppel CL, and Lesnefsky EJ. Ischemic defects in the electron transport chain increase the production of reactive oxygen species from isolated rat heart mitochondria. Am J Physiol Cell Physiol 294: C460-466, 2008.

40. Chen V, lanuzzo CD, Fong BC, and Spitzer JJ. The effects of acute and chronic diabetes on myocardial metabolism in rats. Diabetes 33: 1078-1084, 1984.

41. Chicco AJ and Sparagna GC. Role of cardiolipin alterations in mitochondrial dysfunction and disease. Am J Physiol Cell Physiol 292: C33-44, 2007.

42. Choi KM, Zhong Y, Hoit BD, Grupp IL, Hahn H, Dilly KW, Guatimosim S, Lederer WJ, and Matlib MA. Defective intracellular $\mathrm{Ca}(2+)$ signaling contributes to cardiomyopathy in Type 1 diabetic rats. Am J Physiol Heart Circ Physiol 283: H13981408, 2002.

43. Clearinghouse NDI. National Diabetes Statistics, 2011.

44. Coleman DL. Obese and diabetes: two mutant genes causing diabetes-obesity syndromes in mice. Diabetologia 14: 141-148, 1978.

45. Control CfD. National Diabetes Fact Sheet. 2011.

46. Dabkowski ER. Quantitative proteomic analysis of distinct mitochondrial subpopulations in diabetic myocardium. FASEB J 22, 2008.

47. Dabkowski ER, Baseler WA, Williamson CL, Powell M, Razunguzwa TT, Frisbee JC, and Hollander JM. Mitochondrial dysfunction in the type 2 diabetic heart is associated with alterations in spatially distinct mitochondrial proteomes. Am J Physiol Heart Circ Physiol 299: H529-540, 2010.

48. Dabkowski ER, Williamson CL, Bukowski VC, Chapman RS, Leonard SS, Peer CJ, Callery PS, and Hollander JM. Diabetic cardiomyopathy-associated 
dysfunction in spatially distinct mitochondrial subpopulations. Am J Physiol Heart Circ Physiol 296: H359-369, 2009.

49. Das AM and Harris DA. Control of mitochondrial ATP synthase in heart cells: inactive to active transitions caused by beating or positive inotropic agents. Cardiovascular Research 24: 411-417, 1990.

50. de Kroon AIPM, Dolis D, Mayer A, Lill R, and de Kruijff B. Phospholipid composition of highly purified mitochondrial outer membranes of rat liver and Neurospora crassa. Is cardiolipin present in the mitochondrial outer membrane? Biochimica et Biophysica Acta (BBA) - Biomembranes 1325: 108-116, 1997.

51. Devereux RB, Roman MJ, Paranicas M, OÂ'Grady MJ, Lee ET, Welty TK, Fabsitz RR, Robbins D, Rhoades ER, and Howard BV. Impact of Diabetes on Cardiac Structure and Function : The Strong Heart Study. Circulation 101: 2271-2276, 2000.

52. Droge W. Free radicals in the physiological control of cell function. Physiol Rev 82: 47-95, 2002.

53. Drummen GPC, van Liebergen LCM, Op den Kamp JAF, and Post JA. C11BODIPY581/591, an oxidation-sensitive fluorescent lipid peroxidation probe: (micro)spectroscopic characterization and validation of methodology. Free Radical Biology and Medicine 33: 473-490, 2002.

54. Duchen MR. Mitochondria and calcium: from cell signalling to cell death. The Journal of Physiology 529: 57-68, 2000. 
55. Engkilde K, Buschard K, Hansen AK, Menne T, and Johansen JD. Prevention of diabetes in NOD mice by repeated exposures to a contact allergen inducing a sub-clinical dermatitis. PLoS One 5: e10591, 2010.

56. Epstein PN, Overbeek PA, and Means AR. Calmodulin-induced early-onset diabetes in transgenic mice. Cell 58: 1067-1073, 1989.

57. Fang ZY, Prins JB, and Marwick TH. Diabetic Cardiomyopathy: Evidence, Mechanisms, and Therapeutic Implications. Endocrine Reviews 25: 543-567, 2004.

58. Fein FS. Diabetic cardiomyopathy. Diabetes Care 13: 1169-1179, 1990.

59. Fein FS, Kornstein LB, Strobeck JE, Capasso JM, and Sonnenblick EH. Altered myocardial mechanics in diabetic rats. Circ Res 47: 922-933, 1980.

60. Fein FS and Sonnenblick EH. Diabetic cardiomyopathy. Cardiovasc Drugs Ther 8: 65-73, 1994.

61. Finck BN, Lehman JJ, Leone TC, Welch MJ, Bennett MJ, Kovacs A, Han X, Gross RW, Kozak R, Lopaschuk GD, and Kelly DP. The cardiac phenotype induced by PPARalpha overexpression mimics that caused by diabetes mellitus. $J$ Clin Invest 109: 121-130, 2002.

62. Flarsheim CE, Grupp IL, and Matlib MA. Mitochondrial dysfunction accompanies diastolic dysfunction in diabetic rat heart. American Journal of Physiology - Heart and Circulatory Physiology 271: H192-H202, 1996.

63. Fry $\mathbf{M}$ and Green DE. Cardiolipin requirement for electron transfer in complex I and III of the mitochondrial respiratory chain. Journal of Biological Chemistry 256: 18741880, 1981. 
64. Galderisi M. Diastolic dysfunction and diabetic cardiomyopathy: evaluation by Doppler echocardiography. J Am Coll Cardiol 48: 1548-1551, 2006.

65. Galderisi M, Anderson KM, Wilson PW, and Levy D. Echocardiographic evidence for the existence of a distinct diabetic cardiomyopathy (the Framingham Heart Study). Am J Cardiol 68: 85-89, 1991.

66. Garcia MJ, McNamara PM, Gordon T, and Kannel WB. Morbidity and mortality in diabetics in the Framingham population. Sixteen year follow-up study. Diabetes 23 : 105-111, 1974.

67. Ghosh S, Pulinilkunnil T, Yuen G, Kewalramani G, An D, Qi D, Abrahani A, and Rodrigues B. Cardiomyocyte apoptosis induced by short-term diabetes requires mitochondrial GSH depletion. Am J Physiol Heart Circ Physiol 289: H768-776, 2005.

68. Green K, Brand MD, and Murphy MP. Prevention of Mitochondrial Oxidative Damage as a Therapeutic Strategy in Diabetes. Diabetes 53: S110-S118, 2004.

69. Green K, Brand MD, and Murphy MP. Prevention of mitochondrial oxidative damage as a therapeutic strategy in diabetes. Diabetes 53 Suppl 1: S110-118, 2004.

70. Grundy SM, Benjamin IJ, Burke GL, Chait A, Eckel RH, Howard BV, Mitch W, Smith SC, Jr., and Sowers JR. Diabetes and cardiovascular disease: a statement for healthcare professionals from the American Heart Association. Circulation 100: 1134-1146, 1999.

71. Gupta RS. Evolution of the chaperonin families (Hsp60, Hsp10 and Tcp-1) of proteins and the origin of eukaryotic cells. Mol Microbiol 15: 1-11, 1995.

72. Haines TH and Dencher NA. Cardiolipin: a proton trap for oxidative phosphorylation. FEBS Letters 528: 35-39, 2002. 
73. Han X, Yang J, Cheng H, Yang K, Abendschein DR, and Gross RW. Shotgun lipidomics identifies cardiolipin depletion in diabetic myocardium linking altered substrate utilization with mitochondrial dysfunction. Biochemistry 44: 16684-16694, 2005.

74. Hansford RG and Zorov D. Role of mitochondrial calcium transport in the control of substrate oxidation. Mol Cell Biochem 184: 359-369, 1998.

75. Hardie DG. Minireview: the AMP-activated protein kinase cascade: the key sensor of cellular energy status. Endocrinology 144: 5179-5183, 2003.

76. Hattori Y, Matsuda N, Kimura J, Ishitani T, Tamada A, Gando S, Kemmotsu

O, and Kanno M. Diminished function and expression of the cardiac $\mathrm{Na}+-\mathrm{Ca}+$ exchanger in diabetic rats: implication in Ca2+ overload. J Physiol 527 Pt 1: 85-94, 2000.

77. Herlein JA, Fink BD, O'Malley Y, and Sivitz WI. Superoxide and respiratory coupling in mitochondria of insulin-deficient diabetic rats. Endocrinology 150: 46-55, 2009.

78. Hexeberg S, Hessevik I, and Hexeberg E. Intravenous lipid infusion results in myocardial lipid droplet accumulation combined with reduced myocardial performance in heparinized rabbits. Acta Physiol Scand 153: 159-168, 1995.

79. Holmuhamedov EL, Oberlin A, Short K, Terzic A, and Jahangir A. Cardiac Subsarcolemmal and Interfibrillar Mitochondria Display Distinct Responsiveness to Protection by Diazoxide. PLoS One 7: e44667, 2012.

80. Hoppel CL, Moghaddas S, and Lesnefsky EJ. Interfibrillar cardiac mitochondrial comples III defects in the aging rat heart. Biogerontology 3: 41-44, 2002. 
81. Horman S, Browne G, Krause U, Patel J, Vertommen D, Bertrand L, Lavoinne A, Hue L, Proud C, and Rider M. Activation of AMP-activated protein kinase leads to the phosphorylation of elongation factor 2 and an inhibition of protein synthesis. Curr Biol 12: 1419-1423, 2002.

82. Houtkooper R and Vaz F. Cardiolipin, the heart of mitochondrial metabolism. Cellular and Molecular Life Sciences 65: 2493-2506, 2008.

83. Hovius R, Lambrechts H, Nicolay K, and de Kruijff B. Improved methods to isolate and subfractionate rat liver mitochondria. Lipid composition of the inner and outer membrane. Biochim Biophys Acta 1021: 217-226, 1990.

84. How OJ, Aasum E, Severson DL, Chan WY, Essop MF, and Larsen TS. Increased myocardial oxygen consumption reduces cardiac efficiency in diabetic mice. Diabetes 55: 466-473, 2006.

85. Imai H and Nakagawa Y. Biological significance of phospholipid hydroperoxide glutathione peroxidase (PHGPx, GPx4) in mammalian cells. Free Radic Biol Med 34: 145-169, 2003.

86. Indiveri C, Tonazzi A, Prezioso G, and Palmieri F. Kinetic characterization of the reconstituted carnitine carrier from rat liver mitochondria. Biochim Biophys Acta 1065: 231-238, 1991.

87. Jackson CV, McGrath GM, Tahiliani AG, Vadlamudi RV, and McNeill JH. A functional and ultrastructural analysis of experimental diabetic rat myocardium. Manifestation of a cardiomyopathy. Diabetes 34: 876-883, 1985. 
88. Joost H-G, Al-Hasani H, Schurmann A, Bortell R, and Yang C. The BB Rat as a Model of Human Type 1 Diabetes. In: Animal Models in Diabetes Research: Humana Press, 2012, p. 31-44.

89. Kadenbach B, Mende P, Kolbe HV, Stipani I, and Palmieri F. The mitochondrial phosphate carrier has an essential requirement for cardiolipin. FEBS Lett 139: 109-112, 1982.

90. Kajstura J, Fiordaliso F, Andreoli AM, Li B, Chimenti S, Medow MS, Limana F, Nadal-Ginard B, Leri A, and Anversa P. IGF-1 overexpression inhibits the development of diabetic cardiomyopathy and angiotensin II-mediated oxidative stress. Diabetes 50: 1414-1424, 2001.

91. Kashihara H, Shi ZQ, Yu JZ, McNeill JH, and Tibbits GF. Effects of diabetes and hypertension on myocardial Na+-Ca2+ exchange. Can J Physiol Pharmacol 78: 1219, 2000.

92. Kaul SC, Wadhwa R, Baseler W, Croston T, and Hollander J. Functional Characteristics of Mortalin. In: Mortalin Biology: Life, Stress and Death: Springer Netherlands, 2012, p. 55-80.

93. Kikutani $\mathbf{H}$ and Makino S. The murine autoimmune diabetes model: NOD and related strains. Adv Immunol 51: 285-322, 1992.

94. Kim J, Minkler PE, Salomon RG, Anderson VE, and Hoppel CL. Cardiolipin: characterization of distinct oxidized molecular species. Journal of Lipid Research, 2010. 95. King $\mathbf{C}$ and Sarvetnick $\mathbf{N}$. The incidence of type-1 diabetes in NOD mice is modulated by restricted flora not germ-free conditions. PLoS One 6: e17049, 2011. 
96. King KL, Young ME, Kerner J, Huang H, O'Shea KM, Alexson SE, Hoppel

CL, and Stanley WC. Diabetes or peroxisome proliferator-activated receptor alpha agonist increases mitochondrial thioesterase I activity in heart. J Lipid Res 48: 15111517, 2007.

97. Lesnefsky EJ, Chen Q, Slabe TJ, Stoll MSK, Minkler PE, Hassan MO, Tandler B, and Hoppel CL. Ischemia, rather than reperfusion, inhibits respiration through cytochrome oxidase in the isolated, perfused rabbit heart: role of cardiolipin. Am J Physiol Heart Circ Physiol 287: H258-267, 2004.

98. Lesnefsky EJ, Moghaddas S, Tandler B, Kerner J, and Hoppel CL. Mitochondrial dysfunction in cardiac disease: ischemia--reperfusion, aging, and heart failure. J Mol Cell Cardio/ 33: 1065-1089, 2001.

99. Li SY, Yang X, Ceylan-Isik AF, Du M, Sreejayan N, and Ren J. Cardiac contractile dysfunction in Lep/Lep obesity is accompanied by NADPH oxidase activation, oxidative modification of sarco(endo)plasmic reticulum $\mathrm{Ca} 2+-\mathrm{ATPase}$ and myosin heavy chain isozyme switch. Diabetologia 49: 1434-1446, 2006.

100. Liu JE, Palmieri V, Roman MJ, Bella JN, Fabsitz R, Howard BV, Welty TK, Lee ET, and Devereux RB. The impact of diabetes on left ventricular filling pattern in normotensive and hypertensive adults: the Strong Heart Study. J Am Coll Cardiol 37: 1943-1949, 2001.

101. Lu B, Xu FY, Jiang YJ, Choy PC, Hatch GM, Grunfeld C, and Feingold KR. Cloning and characterization of a cDNA encoding human cardiolipin synthase (hCLS1). J Lipid Res 47: 1140-1145, 2006. 
102. Makino S, Kunimoto K, Muraoka Y, Mizushima Y, Katagiri K, and Tochino Y. Breeding of a non-obese, diabetic strain of mice. Jikken Dobutsu 29: 1-13, 1980.

103. Mazumder PK, O'Neill BT, Roberts MW, Buchanan J, Yun UJ, Cooksey RC, Boudina S, and Abel ED. Impaired cardiac efficiency and increased fatty acid oxidation in insulin-resistant ob/ob mouse hearts. Diabetes 53: 2366-2374, 2004.

104. McCormack JG and Denton RM. Mitochondrial Ca2+ transport and the role of intramitochondrial Ca2+ in the regulation of energy metabolism. Dev Neurosci 15: 165173, 1993.

105. Mirsky I. Assessment of diastolic function: suggested methods and future considerations. Circulation 69: 836-841, 1984.

106. Muoio DM, MacLean PS, Lang DB, Li S, Houmard JA, Way JM, Winegar DA, Corton JC, Dohm GL, and Kraus WE. Fatty acid homeostasis and induction of lipid regulatory genes in skeletal muscles of peroxisome proliferator-activated receptor (PPAR) alpha knock-out mice. Evidence for compensatory regulation by PPAR delta. $J$ Biol Chem 277: 26089-26097, 2002.

107. Nalecz KA, Bolli R, Wojtczak L, and Azzi A. The monocarboxylate carrier from bovine heart mitochondria: partial purification and its substrate-transporting properties in a reconstituted system. Biochim Biophys Acta 851: 29-37, 1986.

108. Neupert W and Herrmann JM. Translocation of proteins into mitochondria. Annu Rev Biochem 76: 723-749, 2007.

109. Nichols GA, Hillier TA, Erbey JR, and Brown JB. Congestive heart failure in type 2 diabetes: prevalence, incidence, and risk factors. Diabetes Care 24: 1614-1619, 2001. 
110. Nielsen J, Mogensen M, Vind BF, Sahlin K, Hojlund K, Schroder HD, and Ortenblad N. Increased subsarcolemmal lipids in type 2 diabetes: effect of training on localization of lipids, mitochondria, and glycogen in sedentary human skeletal muscle. Am J Physiol Endocrinol Metab 298: E706-713, 2010.

111. Nishimura RA and Tajik AJ. Evaluation of diastolic filling of left ventricle in health and disease: Doppler echocardiography is the clinician's Rosetta Stone. J Am Coll Cardiol 30: 8-18, 1997.

112. Ohtake T, Yokoyama I, Watanabe T, Momose T, Serezawa T, Nishikawa J, and Sasaki Y. Myocardial glucose metabolism in noninsulin-dependent diabetes mellitus patients evaluated by FDG-PET. J Nucl Med 36: 456-463, 1995.

113. Oliveira PJ, Seica R, Coxito PM, Rolo AP, Palmeira CM, Santos MS, and Moreno AJ. Enhanced permeability transition explains the reduced calcium uptake in cardiac mitochondria from streptozotocin-induced diabetic rats. FEBS Lett 554: 511$514,2003$.

114. Oliveira PJ, Seica R, Santos DL, Rolo AP, Sardao VA, Ferreira FM, Palmeira CM, Santos MS, and Moreno AJ. Vitamin E or coenzyme Q10 administration is not fully advantageous for heart mitochondrial function in diabetic goto kakizaki rats. Mitochondrion 3: 337-345, 2004.

115. Orrenius S, Gogvadze V, and Zhivotovsky B. Mitochondrial oxidative stress: implications for cell death. Annu Rev Pharmacol Toxicol 47: 143-183, 2007.

116. Palmer JW, Tandler B, and Hoppel CL. Biochemical properties of subsarcolemmal and interfibrillar mitochondria isolated from rat cardiac muscle. Journal of Biological Chemistry 252: 8731-8739, 1977. 
117. Paradise NF, Pilati CF, Payne WR, and Finkelstein JA. Left ventricular function of the isolated, genetically obese rat's heart. Am J Physiol 248: H438-444, 1985.

118. Penpargkul S, Schaible T, Yipintsoi T, and Scheuer J. The effect of diabetes on performance and metabolism of rat hearts. Circ Res 47: 911-921, 1980.

119. Petrosillo G, Ruggiero FM, and Paradies G. Role of reactive oxygen species and cardiolipin in the release of cytochrome c from mitochondria. FASEB J 17: 22022208, 2003.

120. Rabol R, Boushel R, and Dela F. Mitochondrial oxidative function and type 2 diabetes. Appl Physiol Nutr Metab 31: 675-683, 2006.

121. Regan TJ, Ettinger PO, Khan MI, Jesrani MU, Lyons MM, Oldewurtel HA, and Weber M. Altered Myocardial Function and Metabolism in Chronic Diabetes Mellitus without Ischemia in Dogs. Circulation Research 35: 222-237, 1974.

122. Regan TJ, Lyons MM, Ahmed SS, Levinson GE, Oldewurtel HA, Ahmad MR, and Haider B. Evidence for cardiomyopathy in familial diabetes mellitus. J Clin Invest 60: 884-899, 1977.

123. Ritov VB, Menshikova EV, He J, Ferrell RE, Goodpaster BH, and Kelley DE. Deficiency of subsarcolemmal mitochondria in obesity and type 2 diabetes. Diabetes 54 : 8-14, 2005.

124. Riva A, Tandler B, Loffredo F, Vazquez E, and Hoppel C. Structural differences in two biochemically defined populations of cardiac mitochondria. Am J Physiol Heart Circ Physiol 289: H868-872, 2005. 
125. Robertson RP and Harmon JS. Diabetes, glucose toxicity, and oxidative stress: A case of double jeopardy for the pancreatic islet [beta] cell. Free Radical Biology and Medicine 41: 177-184, 2006.

126. Rodrigues B and McNeill JH. Cardiac dysfunction in isolated perfused hearts from spontaneously diabetic BB rats. Can J Physiol Pharmacol 68: 514-518, 1990.

127. Roger VL, Go AS, Lloyd-Jones DM, Adams RJ, Berry JD, Brown TM, Carnethon MR, Dai S, de Simone G, Ford ES, Fox CS, Fullerton HJ, Gillespie C, Greenlund KJ, Hailpern SM, Heit JA, Ho PM, Howard VJ, Kissela BM, Kittner SJ, Lackland DT, Lichtman JH, Lisabeth LD, Makuc DM, Marcus GM, Marelli A, Matchar DB, McDermott MM, Meigs JB, Moy CS, Mozaffarian D, Mussolino ME, Nichol G, Paynter NP, Rosamond WD, Sorlie PD, Stafford RS, Turan TN, Turner MB, Wong ND, and Wylie-Rosett J. Heart Disease and Stroke Statistics - 2011 Update Circulation 123: e18-e209.

128. Rolo AP and Palmeira CM. Diabetes and mitochondrial function: Role of hyperglycemia and oxidative stress. Toxicology and Applied Pharmacology 212: 167178, 2006.

129. Rolo AP and Palmeira CM. Diabetes and mitochondrial function: role of hyperglycemia and oxidative stress. Toxicol Appl Pharmacol 212: 167-178, 2006.

130. Rosca MG, Vazquez EJ, Kerner J, Parland W, Chandler MP, Stanley W, Sabbah HN, and Hoppel CL. Cardiac mitochondria in heart failure: decrease in respirasomes and oxidative phosphorylation. Cardiovasc Res 80: 30-39, 2008. 
131. Rubler S, Dlugash J, Yuceoglu YZ, Kumral T, Branwood AW, and Grishman

A. New type of cardiomyopathy associated with diabetic glomerulosclerosis. Am J Cardiol 30: 595-602, 1972.

132. Santos DL, Palmeira CM, Seiccedila R, Dias J, Mesquita J, Moreno AJ, and Santos MS. Diabetes and mitochondrial oxidative stress: A study using heart mitochondria from the diabetic Goto-Kakizaki rat. Molecular and Cellular Biochemistry 246: 163-170, 2003.

133. Schlame $\mathbf{M}$, Rua $\mathbf{D}$, and Greenberg $\mathbf{M L}$. The biosynthesis and functional role of cardiolipin. Prog Lipid Res 39: 257-288, 2000.

134. Scognamiglio R, Avogaro A, Negut C, Piccolotto R, Vigili de Kreutzenberg S, and Tiengo A. Early myocardial dysfunction in the diabetic heart: current research and clinical applications. Am J Cardiol 93: 17A-20A, 2004.

135. Sedlak E and Robinson NC. Phospholipase A(2) digestion of cardiolipin bound to bovine cytochrome c oxidase alters both activity and quaternary structure. Biochemistry 38: 14966-14972, 1999.

136. Severson DL. Diabetic cardiomyopathy: recent evidence from mouse models of type 1 and type 2 diabetes. Can J Physiol Pharmacol 82: 813-823, 2004.

137. Shen X, Zheng S, Metreveli NS, and Epstein PN. Protection of cardiac mitochondria by overexpression of MnSOD reduces diabetic cardiomyopathy. Diabetes 55: 798-805, 2006.

138. Shen X, Zheng S, Thongboonkerd V, Xu M, Pierce WM, Jr., Klein JB, and Epstein PN. Cardiac mitochondrial damage and biogenesis in a chronic model of type 1 diabetes. Am J Physiol Endocrinol Metab 287: E896-905, 2004. 
139. Singh B, Patel HV, Ridley RG, Freeman KB, and Gupta RS. Mitochondrial import of the human chaperonin (HSP60) protein. Biochem Biophys Res Commun 169: 391-396, 1990.

140. Singh VP, Le B, Khode R, Baker KM, and Kumar R. Intracellular angiotensin II production in diabetic rats is correlated with cardiomyocyte apoptosis, oxidative stress, and cardiac fibrosis. Diabetes 57: 3297-3306, 2008.

141. Solano MP and Goldberg RB. Lipid Management in Type 2 Diabetes. Clinical Diabetes 24: 27-32, 2006.

142. Song Y, Du Y, Prabhu SD, and Epstein PN. Diabetic Cardiomyopathy in OVE26 Mice Shows Mitochondrial ROS Production and Divergence Between In Vivo and In Vitro Contractility. Rev Diabet Stud 4: 159-168, 2007.

143. Stanley WC and Hoppel CL. Mitochondrial dysfunction in heart failure: potential for therapeutic interventions? Cardiovascular Research 45: 805-806, 2000.

144. Struthers AD and Morris AD. Screening for and treating left-ventricular abnormalities in diabetes mellitus: a new way of reducing cardiac deaths. Lancet 359: 1430-1432, 2002.

145. Szkudelski T. The mechanism of alloxan and streptozotocin action in B cells of the rat pancreas. Physiol Res 50: 537-546, 2001.

146. Takenaka K, Sakamoto T, Amano K, Oku J, Fujinami K, Murakami T, Toda I, Kawakubo K, and Sugimoto T. Left ventricular filling determined by Doppler echocardiography in diabetes mellitus. Am J Cardiol 61: 1140-1143, 1988. 
147. Tomita M, Mukae S, Geshi E, Umetsu K, Nakatani M, and Katagiri T. Mitochondrial respiratory impairment in streptozotocin-induced diabetic rat heart. Jpn Circ J 60: 673-682, 1996.

148. Torok Z, Demel RA, Leenhouts JM, and de Kruijff B. Presequence-mediated intermembrane contact formation and lipid flow. A model membrane study. Biochemistry 33: 5589-5594, 1994.

149. Turko IV, Li L, Aulak KS, Stuehr DJ, Chang JY, and Murad F. Protein tyrosine nitration in the mitochondria from diabetic mouse heart. Implications to dysfunctional mitochondria in diabetes. J Biol Chem 278: 33972-33977, 2003.

150. Turko IV and Murad F. Quantitative protein profiling in heart mitochondria from diabetic rats. J Biol Chem 278: 35844-35849, 2003.

151. Vadlamudi RV, Rodgers RL, and McNeill JH. The effect of chronic alloxan- and streptozotocin-induced diabetes on isolated rat heart performance. Can $J$ Physiol Pharmacol 60: 902-911, 1982.

152. Vadvalkar SS, Baily CN, Matsuzaki S, West M, Tesiram YA, and Humphries

KM. Metabolic inflexibility and protein lysine acetylation in heart mitochondria of a chronic model of type 1 diabetes. Biochem J 449: 253-261, 2013.

153. Valko M, Leibfritz D, Moncol J, Cronin MTD, Mazur M, and Telser J. Free radicals and antioxidants in normal physiological functions and human disease. The International Journal of Biochemistry \& Cell Biology 39: 44-84, 2007.

154. van de Weijer T, Schrauwen-Hinderling VB, and Schrauwen P. Lipotoxicity in type 2 diabetic cardiomyopathy. Cardiovascular Research 92: 10-18, 2011. 
155. Van Linthout S, Seeland U, Riad A, Eckhardt O, Hohl M, Dhayat N, Richter U, Fischer JW, Bohm M, Pauschinger M, Schultheiss HP, and Tschope C. Reduced MMP-2 activity contributes to cardiac fibrosis in experimental diabetic cardiomyopathy. Basic Res Cardiol 103: 319-327, 2008.

156. Voos W, Martin H, Krimmer T, and Pfanner N. Mechanisms of protein translocation into mitochondria. Biochim Biophys Acta 1422: 235-254, 1999.

157. Weinstein ES, Benson DW, and Fry DE. Subpopulations of human heart mitochondria. Journal of Surgical Research 40: 495-498, 1986.

158. Weiss JS and Sumpio BE. Review of prevalence and outcome of vascular disease in patients with diabetes mellitus. Eur J Vasc Endovasc Surg 31: 143-150, 2006.

159. Wende AR and Abel ED. Lipotoxicity in the heart. Biochimica et Biophysica Acta (BBA) - Molecular and Cell Biology of Lipids 1801: 311-319, 2010.

160. Whalen BJ, Mordes JP, and Rossini AA. The BB Rat as a Model of Human Insulin-Dependent Diabetes Mellitus. In: Current Protocols in Immunology: John Wiley \& Sons, Inc., 2001.

161. Wild S, Roglic G, Green A, Sicree R, and King H. Global prevalence of diabetes: estimates for the year 2000 and projections for 2030. Diabetes Care 27: 10471053, 2004.

162. Williamson CL, Dabkowski ER, Baseler WA, Croston TL, Alway SE, and Hollander JM. Enhanced apoptotic propensity in diabetic cardiac mitochondria: influence of subcellular spatial location. Am J Physiol Heart Circ Physiol 298: H633-642, 2010. 
163. Williamson CL, Dabkowski ER, Dillmann WH, and Hollander JM. Mitochondria protection from hypoxia/reoxygenation injury with mitochondria heat shock protein 70 overexpression. Am J Physiol Heart Circ Physiol 294: H249-256, 2008.

164. Wright JJ, Kim J, Buchanan J, Boudina S, Sena S, Bakirtzi K, Ilkun O, Theobald HA, Cooksey RC, Kandror KV, and Abel ED. Mechanisms for increased myocardial fatty acid utilization following short-term high-fat feeding. Cardiovasc Res 82: 351-360, 2009.

165. Wu KK and Huan Y. Streptozotocin-Induced Diabetic Models in Mice and Rats. In: Current Protocols in Pharmacology: John Wiley \& Sons, Inc., 2001.

166. Yang R and Barouch LA. Leptin Signaling and Obesity: Cardiovascular Consequences. Circulation Research 101: 545-559, 2007.

167. Ye G, Metreveli NS, Ren J, and Epstein PN. Metallothionein prevents diabetesinduced deficits in cardiomyocytes by inhibiting reactive oxygen species production. Diabetes 52: 777-783, 2003.

168. Yoshioka M, Kayo T, Ikeda T, and Koizumi A. A novel locus, Mody4, distal to D7Mit189 on chromosome 7 determines early-onset NIDDM in nonobese C57BL/6 (Akita) mutant mice. Diabetes 46: 887-894, 1997.

169. Young ME, Guthrie PH, Razeghi P, Leighton B, Abbasi S, Patil S, Youker KA, and Taegtmeyer H. Impaired long-chain fatty acid oxidation and contractile dysfunction in the obese Zucker rat heart. Diabetes 51: 2587-2595, 2002.

170. Yue P, Arai T, Terashima M, Sheikh AY, Cao F, Charo D, Hoyt G, Robbins RC, Ashley EA, Wu J, Yang PC, and Tsao PS. Magnetic resonance imaging of 
progressive cardiomyopathic changes in the db/db mouse. Am J Physiol Heart Circ Physiol 292: H2106-2118, 2007.

171. Zarich SW, Arbuckle BE, Cohen LR, Roberts M, and Nesto RW. Diastolic abnormalities in young asymptomatic diabetic patients assessed by pulsed Doppler echocardiography. J Am Coll Cardiol 12: 114-120, 1988.

172. Zhang Y, Proenca R, Maffei M, Barone M, Leopold L, and Friedman JM. Positional cloning of the mouse obese gene and its human homologue. Nature 372: 425-432, 1994. 


\section{Chapter 2:}

\section{Evaluation of the cardiolipin biosynthetic pathway and its interactions in the diabetic heart}

As accepted in Life Sciences, 2013 July

Tara L. Croston ${ }^{1}$, Danielle L. Shepherd ${ }^{1}$, Dharendra Thapa ${ }^{1}$, Cody E. Nichols ${ }^{1}$, Sara E. Lewis $^{1}$, Erinne R. Dabkowski ${ }^{1}$, Rajaganapathi Jagannathan ${ }^{1}$, Walter A. Baseler ${ }^{1}$, and John M. Hollander ${ }^{1}$

${ }^{1}$ West Virginia University School of Medicine, Division of Exercise Physiology; Center for Cardiovascular and Respiratory Sciences; Morgantown, WV 26506

Running Title: Cardiolipin Biosynthesis in the Diabetic Heart 


\section{ABSTRACT}

Aims: We have previously reported alterations in cardiolipin content and inner mitochondrial membrane (IMM) proteomic make-up specifically in interfibrillar mitochondria (IFM) in the type 1 diabetic heart; however, the mechanism underlying this alteration is unknown. The goal of this study was to determine how the cardiolipin biosynthetic pathway and cardiolipin-IMM protein interactions are impacted by type 1 diabetes mellitus.

Main methods: Male FVB mice were made diabetic by multiple low-dose streptozotocin injections and sacrificed five weeks post-diabetic onset. Messenger RNA was measured and cardiac mitochondrial subpopulations were isolated. Further mitochondrial functional experimentation included evaluating the protein expression of the enzymes directly responsible for cardiolipin biosynthesis, as well as ATP synthase activity. Interactions between cardiolipin and ATP synthase subunits were also examined.

Key findings: Western blot analysis revealed a significant decrease of cardiolipin synthase (CRLS) protein content in diabetic IFM, with a concomitant decrease in its activity. ATP synthase activity was also significantly decreased. We identified two novel direct interactions between two subunits of the ATP synthase $F_{0}$ complex (ATP5F1 and ATP5H), both of which were significantly decreased in diabetic IFM.

Significance: Overall, these results indicate that type 1 diabetes mellitus negatively impacts the cardiolipin biosynthetic pathway specifically at CRLS, contributing to decreased cardiolipin content and loss of interactions with key ATP synthase $F_{0}$ complex constituents in the IFM. 
KEY WORDS: diabetes mellitus, mitochondria, inner mitochondria membrane, cardiolipin, ATP Synthase 


\section{INTRODUCTION}

Cardiovascular complications are the primary cause of morbidity in diabetic patients, of which diabetic cardiomyopathy has been associated with mitochondrial dysfunction $(10,28,31)$. The inner mitochondria membrane (IMM) houses vital processes in a specific lipid environment. Alteration in the IMM lipid environment has been associated with dysfunction to mitochondrial processes $(1,6,14,18)$. Proteomic analyses of type 1 diabetic mitochondria indicates that IMM proteins may be particularly prone to damage and loss (3).

Impaired mitochondrial function, particularly in those processes situated in the IMM, may be associated with disruption to the phospholipid environment contained within. Most abundant in the mammalian heart, cardiolipin is a unique phospholipid, primarily located in the IMM (26) and is thought to play a critical role in mitochondrial structure and bioenergetics (1). The biosynthetic pathway (Figure 2.1) begins in the outer mitochondrial membrane where phosphatidic acid is converted into cytidine diphosphate diacylglycerol (CDP-DAG) by cytidine diphosphate diacylglycerol synthase (CDS) (17). Phosphatidylglycerol synthase (PGS) then converts CDP-DAG to phosphatidylglycerol phosphate (PG-P), where the phosphate is removed by phosphatidylglycerol phosphatase (PGP), resulting in the formation of phosphatidylglycerol (PG). Cardiolipin synthase (CRLS) then catalyzes the condensation of CDP-DAG and PG, resulting in a nascent form of cardiolipin $(6,21)$. Because the nascent cardiolipin does not contain the precise fatty acyl side chains for 
the specific tissue, a remodeling process takes place, where tafazzin (TAZ) or monolysocardiolipin acyltransferase, converts the nascent cardiolipin to mature cardiolipin containing specific fatty acyl side chains (6), which is dependent upon the tissue and environment. Linoleic acid, the most abundant fatty acid found in cardiac mitochondria, constitutes approximately $80 \%$ of all side chains $(2,26)$. The properties of cardiolipin allow it to interact with IMM proteins and facilitate proper mitochondrial function.

Literature suggests an interaction between cardiolipin and the electron transport chain (ETC) complexes involved in oxidative phosphorylation (26), including complexes I, III, IV, and ATP synthase $(12,27)$. Cardiolipin plays a critical role in the organization of mammalian mitochondrial ATP synthase and it has been suggested that the $F_{0}$ complex of ATP synthase contains high affinity binding sites for cardiolipin (1); however, the specific binding sites remain undefined. Cardiolipin also serves as a proton reservoir for maintenance of IMM potential thus, contributing to ATP synthesis (13). Shotgun lipidomics revealed decreased tetralinoleic cardiolipin content in diabetic hearts suggesting a possible mechanistic reliance on the interaction between IMM protein activity and cardiolipin (14). We have previously reported decreased ETC protein content and function in IFM following a type 1 diabetic insult $(3,8)$, correlating with decreased tetralinoleic cardiolipin content, with no effect on subsarcolemmal mitochondria (SSM) situated beneath the plasma membrane (8). 
To date, the impact of type 1 diabetes mellitus on the cardiolipin biosynthetic pathway in mitochondrial subpopulations and on the interactions between cardiolipin and IMM proteins has not been studied. We hypothesized that the decreased cardiolipin content associated with type 1 diabetes mellitus results from defective cardiolipin biosynthesis influencing its association with IMM proteins, with the effects being most pronounced in IFM. 


\section{MATERIALS AND METHODS}

\section{Experimental animals and induction of diabetes}

The animal experiments in this study conformed to the National Institutes of Health (NIH) Guidelines for the Care and Use of Laboratory Animals and were approved by the West Virginia University Animal Care and Use Committee. Male FVB mice were housed in the West Virginia University Health Sciences Center animal facility on a 12$\mathrm{hf}$ light/dark cycle in a temperature controlled room. Mice were given unlimited access to a standard rodent diet and water. Type 1 diabetes mellitus was induced in 6-week-old mice following the protocol of the Animal Models of Diabetic Complications Consortium using multiple low-dose streptozotocin (STZ; Sigma-Aldrich Corporation, St. Louis, MO) intraperitoneal injections. The STZ-induced type 1 diabetic model was chosen because it is the most widely utilized model and the multiple low dose administration is not associated with many of the deleterious side effects observed with the single high-dose approach (34). Injections of $50 \mathrm{mg} / \mathrm{kg}$ body weight STZ dissolved in sodium citrate buffer $(\mathrm{pH} 4.5)$ were performed daily for 5 consecutive days after 6 hours of fasting. Mice serving as vehicle controls were given the same volume per body weight of sodium citrate buffer. One week post-injection, hyperglycemia was confirmed by measuring blood glucose (Contour Blood Glucose test strips; Bayer, Mishawaka, IN), where $>250 \mathrm{mg} / \mathrm{dL}$ was considered diabetic. Five weeks after the onset of hyperglycemia, animals were sacrificed for experimentation. Blood glucose levels were again tested at this time and all remained $>250 \mathrm{mg} / \mathrm{dL}$. 


\section{Individual Mitochondrial Subpopulations Isolation}

Five weeks post-diabetic onset, FVB mice and their littermate controls were sacrificed and hearts excised. Hearts were rinsed in phosphate buffered saline (PBS, $\mathrm{pH}$ 7.4), then blotted dry. SSM and IFM were isolated as previously described by Palmer et al. (25) with modifications by our laboratory $(8,9,33)$. Mitochondrial pellets were resuspended in either $\mathrm{KME}$ buffer $(100 \mathrm{mM} \mathrm{KCl}, 50 \mathrm{mM}$ MOPS, and $0.5 \mathrm{mM}$ EDTA) for Western blot analysis and activity measurements, or in $1.0 \%$ NP-40 wash/binding buffer (10 mM HEPES (pH 7.4), 1.0\% NP-40, $150 \mathrm{mM} \mathrm{NaCl)}$ for cardiolipin/protein interactions. Protein content was determined by the Bradford method using bovine serum albumin as a standard (4).

\section{Cardiolipin}

Isolated mitochondrial subpopulations from control and diabetic hearts were pooled, and sent off to Avanti Polar Lipids, Inc. (Alabaster, AL) for cardiolipin analysis. Extraction and analysis of cardiolipin species were based on the protocols of Sparagna et al. (29) and Minkler et al. (23). In brief, cardiolipin detection was performed by liquid chromatography (Dionex U-3000 binary HPLC) / hybrid tandem mass spectrometry using a quadrapole -linear ion trap (API 4000 Qtrap) operated in the negative ion mode. Mass spectrometer instrument conditions included a spray voltage of $-4.5 \mathrm{kV}$, capillary voltage of $-150 \mathrm{~V}$, heated capillary temperature of $400^{\circ} \mathrm{C}$, and a sheath gas flow rate of 10 arbitrary units. Mitochondrial cardiolipin and internal standard spectra were identified by multiple reaction monitoring. All experiments were performed in triplicate. 


\section{mRNA Isolation and analysis}

mRNA levels were examined as previously described (3). Briefly, hearts were rinsed in phosphate buffered saline (PBS, pH 7.4) then snap frozen in liquid nitrogen. Thirty milligrams of tissue was excised from control and diabetic hearts from which the mRNA was extracted using an RNeasy mini kit, per manufacturer's instructions (Qiagen, Valencia, CA) and reverse transcribed. Equal amounts of cDNA from the hearts of control and diabetic mice were subjected to real-time PCR. Custom primers were designed for target genes CDS, PGS, CRLS, and TAZ. SYBR Green I was used for quantification of respective cDNA replicates (Qiagen, Velencia, CA). Data were normalized by gene expression relative to the levels of GAPDH.

\section{Western Blot Analyses}

Sodium dodecyl sulfate-polyacrylamide gel electrophoresis (SDS-PAGE) was run on a $4-12 \%$ gel as previously described $(20,33)$ with equal amounts of protein loaded for each study treatment. Relative amounts of subpopulation-specific CDS, PGS, CRLS, and TAZ, were assessed using the following primary antibodies; anti-CDS rabbit antibody (product No. ab84019, Abcam, Cambridge, MA), anti-PGS rabbit antibody (product No. ab104815, Abcam, Cambridge, MA), anti-CRLS rabbit antibody (product No. 49444_P050, Aviva Systems Biology, San Diego, CA), and anti-TAZ rabbit antibody (product No. ab84927, Abcam, Cambridge, MA). The secondary antibody used was a goat anti-rabbit IgG horseradish peroxidase conjugate (product No. 10004301, Cayman Chemical Company, Ann Arbor, MI). Detection of signal was performed according to the Pierce ECL Western Blotting Substrate detection system 
manufacturer's directions (Thermo Fisher Scientific Inc., Rockford, IL). Cytochrome $c$ oxidase (COX IV) was used to control for protein loading (product No. ab16056, Abcam, Cambridge, MA). Quantification of chemiluminescent signals were assessed using a G:Box Bioimaging System (Syngene, Frederick, MD), and the data captured using GeneSnap software (Syngene, Frederick, MD). Densitometry was measured using Image J Software (National Institutes of Health, Bethesda, MD).

\section{Cardiolipin Synthase (CRLS) Activity}

CRLS activity was assessed as previously described (5) with minor modifications. Briefly, a reaction mixture containing $20 \mu \mathrm{M}{ }^{14} \mathrm{C}$-oleoyl-CoA (50 $\mathrm{mCi} / \mathrm{mmol}$; American Radiolabeled Chemicals, Inc, MO), 2.0 mM LPG [1-oleoyl-2hydroxy-sn-glycero-3-[phospho-rac-(1-glycerol)]] and 2.0 mM CDP-DAG (Avanti Polar Lipids Inc., Alabaster, AL) were incubated with $50 \mu \mathrm{g}$ of lysed mitochondrial protein for $30 \mathrm{~min}$ at $37^{\circ} \mathrm{C}$. The reactions were terminated by adding chloroform/methanol, followed by a quick vortex, then chloroform, followed by a quick vortex and lastly, $0.1 \mathrm{M} \mathrm{KCl}$ to facilitate phase separation. A brief centrifugation was performed to extract lipids. The organic phase was collected, dried under an argon stream and separated by high performance thin layer chromatography plates (Whatman, GE Healthcare, U.S.A) with chloroform/hexane/methanol/acetic acid (25:15:5:2.5 by volume) as the developing solvent. The high performance thin layer chromatography plates are then stained with iodine vapor. The appropriate lipid spots are scraped off the plate and dissolved in scintillation fluid for scintillation counting. 


\section{ATP Synthase Activity}

Activity was measured in control and diabetic mitochondrial subpopulations as oligomycin-sensitive ATPase activity using an assay coupled with pyruvate kinase which converts the ADP to ATP and produces pyruvate from phosphoenolpyruvate as previously described (7). Protein content was assessed as described above (4) with final values expressed as nanomoles of $\mathrm{NADH}$ per minute per milligram of protein, equal to the nanomoles of $\mathrm{NADH}$ oxidized per minute per milligram of protein.

\section{Lipid-Protein Interaction}

Cardiolipin-coated beads (Product No. P-BCLP, Echelon Biosciences Incorporated, Salt Lake City, UT) were used to determine protein interactions with cardiolipin per the manufacturer's instructions. In brief, $100 \mu$ of cardiolipin-coated beads were incubated with $600 \mu \mathrm{g}$ mitochondrial protein diluted in $1.0 \% \mathrm{NP}-40$ wash/binding buffer overnight at $4^{\circ} \mathrm{C}$. The beads were spun down at $800 \times g$. The supernatant containing the unbound proteins was collected and the beads were washed four times with 10x excess of wash/binding buffer. The proteins bound to the beads were eluted with $4 \mathrm{x}$ Laemmli sample buffer and heated to $95^{\circ} \mathrm{C}$. A Western blot was then performed on the eluted protein from the beads and the unbound protein located in the supernatant using the following specific antibodies: anti-ATP5F1 mouse antibody (product No. ab117991, Abcam, Cambridge, MA), anti-ATP5H mouse antibody (product No. ab110275, Abcam, Cambridge, MA), anti-ATPB mouse antibody (product No. ab14730, Abcam, Cambridge, MA), anti-ATP5A (product No. ab110273, Abcam, Cambridge, MA), and anti-GRP75 rabbit antibody (product No. 2816S, Cell Signaling 
Technology, Danvers, MA). The secondary antibodies used were either a goat antirabbit IgG horseradish peroxidase conjugate (product No. 10004301, Cayman Chemical Company, Ann Arbor, MI) or a goat anti-mouse IgG horseradish peroxidase conjugate (product No. 31430, Thermo Fisher Scientific Inc., Rockford, IL). Detection of signal was performed according to the Pierce ECL Western Blotting Substrate detection system manufacturer's directions (Thermo Fisher Scientific Inc., Rockford, IL). Quantification of chemiluminescent signals were assessed using a G:Box Bioimaging System (Syngene, Frederick, MD), and the data captured using GeneSnap software (Syngene, Frederick, MD) as above. Densitometry was measured using Image J Software (National Institutes of Health, Bethesda, MD) as above. The association between cardiolipin and the mitochondrial protein of interest was determined by using the densitometric ratio of the protein bound to native cardiolipin/protein bound to native cardiolipin + protein bound to the cardiolipin-coated beads.

\section{Statistics}

Means and SEMs were calculated for all data sets. Data were analyzed using a Student's $t$-test (GraphPad Software Inc., La Jolla, CA). Differences between control and diabetic groups were considered statistically significant when a $P<0.05$ was observed. 


\section{RESULTS}

\section{Cardiolipin Content in the Diabetic Heart}

Han et al. (14) indicated an overall decrease in cardiolipin content in the diabetic heart and our laboratory has previously reported decreased tetralinoleic cardiolipin content in the diabetic IFM (8). Because CRLS synthesizes the nascent form of cardiolipin, total cardiolipin content was measured. The cardiolipin content in the IFM significantly decreased from $84.46 \pm 1.93 \mathrm{nmol} / \mathrm{mg}$ of protein in the control heart to $48.75 \pm 2.83 \mathrm{nmol} / \mathrm{mg}$ of protein in the diabetic heart, with no difference in the SSM $(44.99 \pm 1.75 \mathrm{nmol} / \mathrm{mg}$ of protein in control heart compared to $37.87 \pm 0.11 \mathrm{nmol} / \mathrm{mg}$ of protein in diabetic heart); therefore, we hypothesized that the decrease in cardiolipin may be caused by an alteration in the cardiolipin biosynthetic pathway during a diabetic insult.

\section{mRNA Analyses of Cardiolipin Biosynthesis Pathway Constituents}

To gain insight into the impact of diabetes mellitus on transcriptional regulation of the enzymatic components of the cardiolipin biosynthetic pathway, the mRNA levels of three constituent enzymes, CDS, PGS, and CRLS, as well as the remodeling enzyme TAZ, were analyzed. The results indicated no significant differences between control and diabetic heart for any of the constituents responsible for cardiolipin biosynthesis or remodeling (Table 2.1). These results suggest that the observed decreased in IFM cardiolipin content resulting from a type 1 diabetic insult does not occur at the transcriptional level. 
The expression levels of other IMM proteins were also listed in Table 1. None of the mRNA levels of proteins involved in protein import were altered in a diabetic heart. Of all the transcripts involved in oxidative phosphorylation, only electron transfer flavoprotein-ubiquinone oxidoreductase was significantly decreased in the diabetic. This protein is responsible for the transferring of electrons to the mitochondrial respiratory chain. This decrease suggests a reduction in the electron flux through the respiratory chain, which could potentially cause decreased oxidative phosphorylation. This potential result is supported by decreased mitochondrial respiration in the presence of type 1 diabetes mellitus reported by our laboratory (Dabkowski et al. 2009).

\section{Protein Analyses of Cardiolipin Biosynthesis Pathway Constituents}

To determine the impact of diabetes mellitus on translational regulation of the enzymatic components of the cardiolipin biosynthetic pathway, the protein levels of three constituent enzymes, CDS, PGS, and CRLS, as well as the remodeling enzyme TAZ, were analyzed. Western blot analyses revealed a significant decrease only in CRLS protein content in diabetic IFM as compared to control, with no impact on CRLS protein content in the SSM (Figures 2.2E and 2.2F). No significant differences were observed in the protein levels of the other cardiolipin biosynthetic enzymes assessed, regardless of subpopulation (Figure 2.2). It is important to note that CDS-2 is the major isoform in the heart and when the protein expression was measured, there was no difference between the two treatment groups for either subpopulation (data not shown). These results indicate that the observed decrease in IFM cardiolipin content resulting 
from a type 1 diabetic insult is associated with a decrease in the protein content of CRLS, and thus the biosynthetic pathway.

\section{Enzymatic Activity Analyses of CRLS}

Because CRLS protein content was the only change observed in the biosynthetic pathway following a diabetic insult, we investigated the impact of diabetes mellitus on CRLS enzymatic activity using an assay that utilizes ${ }^{14} \mathrm{C}$-oleoyl-coenzyme $\mathrm{A}$ as an acyl donor. ${ }^{14}$ C-oleoyl-coenzyme A lipid was incubated with additional compounds and mitochondrial protein then subjected to high performance thin layer chromatography. Our results revealed a decrease in CRLS activity in diabetic IFM as compared to control (Figure 2.3B), with no change in the SSM (Figure 2.3A). During high performance thin layer chromatography, PG runs below cardiolipin, so as a control to ensure that the assay was only affecting the radioactivity incorporated into cardiolipin, the amount of radioactivity incorporated into PG was also measured through scintillation counting. The results indicated no change in ${ }^{14} \mathrm{C}-\mathrm{PG}$ in diabetic mitochondria relative to control for either subpopulation (Figures $2.3 \mathrm{C}$ and 2.3D). These results complement the CRLS proteomic data and suggest that loss of CRLS protein in the IFM results in a decrease in CRLS enzymatic activity.

\section{ATP Synthase Activity Analyses}

Literature suggests that cardiolipin interacts with components of the ATP synthase complex $(1,11)$; however, these interaction sites are unknown. Further, previous proteomic data from our laboratory indicates that ATP synthase components 
are decreased in diabetic IFM (3). As a result, we determined whether the activity of the ATP synthase complex was impacted in diabetic mitochondria. Our analyses indicated that ATP synthase activity was significantly decreased in the diabetic IFM as compared to control (Figure 2.4B) with no differences observed in the SSM subpopulation (Figure 2.4A). These results suggest that the decrease in ATP synthase activity observed in diabetic IFM is associated with a decrease in cardiolipin content, which may be influencing its functional properties.

\section{Cardiolipin and ATP Synthase Interaction}

To gain insight into which ATP synthase constituent proteins may be interacting with cardiolipin, we developed an approach in which we incubated cardiolipin-coated beads with mitochondrial proteins and probed for the interaction. Figure $2.5 \mathrm{~A}$ and $2.5 \mathrm{~B}$ demonstrates direct association of cardiolipin with ATP synthase subunit b (ATP5F1) and ATP synthase subunit d (ATP5H), both of which are subunits of the $F_{0}$ complex, or proton pore, of the ATP synthase. To validate that the cardiolipin-coated beads were specifically binding to proteins that interact with cardiolipin, interactions of proteins not associated with cardiolipin were examined. First, ATP synthase alpha (ATP5A) and ATP synthase beta (ATPB) were measured. The results indicated that neither protein was interacting with the cardiolipin-coated beads (Figures 2.5C and 2.5D, respectively). Next, glucose regulated protein 75 (GRP75), a protein located in the mitochondrial matrix, was examined. The results indicated the absence of GRP75 bound to the cardiolipin-coated beads in both subpopulations (Figures 2.5E and 2.5F, respectively). 
Once the interactions between the aforementioned ATP synthase subunits and cardiolipin were detected, we quantified the association between the lipid and protein that occurs in the mitochondrion during a diabetic insult. By assessing proteins that are associated with native cardiolipin, which are incapable of binding to the coated beads (native bound), as well as those proteins that can bind to the beads (bead bound), we were able to determine how diabetes mellitus impacts cardiolipin association with these two proteins. Figure 2.6A represents the protein content of ATP5F1 in the different mitochondrial subpopulations of control and diabetic hearts, respectively, while Figure 2.6B represents the protein content of ATP5H. In order to quantify the associations of ATP synthase subunits and native cardiolipin, the optical density of the band that resulted from the protein interacting with native cardiolipin (native bound protein) was divided by the optical density of the bands that resulted from both the protein bound to native cardiolipin (native bound) and the protein bound to the cardiolipin-coated beads (bead bound). This ratio was used to quantify the interactions of cardiolipin with the protein in control and diabetic mitochondrial subpopulations. The results indicated no difference in the associations between cardiolipin and ATP5F1 or ATP5H in the diabetic SSM compared to controls (Figures $2.6 \mathrm{C}$ and 2.6D, respectively). Interestingly, a significant decrease in the interactions between native cardiolipin and both ATP5F1 and ATP5H in diabetic IFM, as compared to control, was observed (Figures 2.6E and 2.6F, respectively). 


\section{DISCUSSION}

Mitochondrial dysfunction is an underlying contributor to diabetic cardiomyopathy $(10,28,31)$. The IMM is an important submitochondrial locale which houses the machinery required for a number of mitochondrial functional processes. When the lipid environment of the IMM is altered, the processes contained within can become impaired $(1,6,14,18)$. Cardiolipin, makes up approximately $20 \%$ of the total IMM phospholipid membrane (32) and is required for proper functioning of a number of mitochondrial processes $(6,12,18)$. In this study, we observed a decrease in total cardiolipin content in the diabetic IFM. Our laboratory has previously reported significant decreases in tetralinoleic cardiolipin content of diabetic IFM, as well as loss of a number of proteins that are constituents for crucial functional processes $(3,8)$. These results are further supported by others who have reported decreased total cardiolipin content 28 days after the induction of diabetes mellitus (14), as well as decreased tetralinoleic cardiolipin in diabetic mouse hearts compared to control days after the induction of diabetes mellitus (15). Thus, our hypothesis for the current study centered on understanding whether

proteomic loss of IMM constituents and cardiolipin content was the result of dysfunction to the pathway responsible for cardiolipin synthesis. If our hypothesis were to be supported, it would suggest a mechanism contributing to mitochondrial dysfunction in the diabetic heart.

The biosynthesis of cardiolipin occurs in the IMM and is a multistep process (Figure 2.1). To determine whether alterations in the cardiolipin biosynthetic pathway 
contribute to loss of cardiolipin, mRNA and protein levels for a number of key constituents were measured. The results indicated no difference in mRNA levels between control and diabetic hearts for any of the enzymes directly responsible for cardiolipin biosynthesis; however, a significant decrease in CRLS protein content was observed in the diabetic IFM. It is unclear why CRLS protein content was decreased specifically in the IFM though the observation may be the result of decreased protein import specifically in the diabetic IFM, which has been reported previously (3).

Because CRLS was the only enzyme affected by a type 1 diabetic insult, we determined whether the response influenced the activity of the CRLS enzyme. Using ${ }^{14} \mathrm{C}$-oleoyl-coenzyme $\mathrm{A}$ as an acyl donor, we measured incorporation of ${ }^{14} \mathrm{C}$ to assess CRLS activity. One limitation to this method is that remodeling of cardiolipin is not taken into account and other methods do not possess this limitation. Nie et al. (24) reported that lysophosphatidylglycerol acyltransferase also acts as a CRLS, converting lyso-PG to PG; therefore, to ensure the CRLS activity assay was only measuring the incorporation of ${ }^{14} \mathrm{C}$ into cardiolipin, we measured the incorporation of ${ }^{14} \mathrm{C}$ into $\mathrm{PG}$. Results indicated no change in ${ }^{14} \mathrm{C}$ - PG levels between diabetic and control samples for either subpopulation, and a significant decrease in ${ }^{14} \mathrm{C}$-cardiolipin levels in diabetic IFM compared to control, with no change in the SSM. These results instill confidence that the methods utilized in our studies are reliable.

Though our data indicated decreased CRLS protein content and activity in the diabetic IFM relative to control with no change observed in the SSM, the protein content 
of CRLS was not different between the SSM and IFM subpopulations; therefore, this eliminated the possibility that absolute CRLS protein levels underlie the decreased CRLS activity observed in the IFM. Hatch et al. (16) reported no change in PG or cardiolipin biosynthesis during a 4 day or 28 day diabetic insult in rats and Taylor et al. (30) suggested that neither cardiolipin content nor CRLS activity was altered during STZ-induced diabetes. These results were obtained by examining total mitochondria as opposed to our current study examining mitochondrial subpopulations, which may explain the different results between studies. In either case, the results support the importance of examining the two distinct pools of mitochondria.

Previous research has indicated an association between cardiolipin and ETC complexes I, III and IV, from which the contact points with cardiolipin have been identified $(12,27)$. Literature has also shown an association between cardiolipin and ATP synthase $(1,11)$. One study demonstrated that cardiolipin is central for the assembly of ATP synthase dimers, affecting the organization and ultimately the structure of the IMM (1). Other studies have suggested that the interaction between cardiolipin and the ATP synthase complex occurs on subunits composing the $F_{0}$ complex of the ATP synthase due to its location within the IMM (11). Although an association between cardiolipin and ATP synthase has been reported, the direct contact points have not been identified. For this reason, we used a novel cardiolipin-coated bead assay to separate out the two pools of specific ATP synthase subunits - those already bound to native cardiolipin and those available to bind to the cardiolipin-coated beads. The ATP synthase subunits predicted to interact with cardiolipin were identified 
using previous proteomic data performed in our laboratory (3). Western blot analysis revealed two direct associations between cardiolipin and ATP synthase.

It is important to note that although the cardiolipin-bead pull-down assay is a quick and accessible method to gather cardiolipin-protein interaction data, the assay requires a large amount of protein. This is a limitation when measuring associations within mitochondrial subpopulations, due to limited yields of mitochondrial subpopulation protein content as compared to the analysis of total mitochondria. Nevertheless, we are the first to detect direct associations between cardiolipin and two specific ATP synthase subunits.

To gain insight into whether cardiolipin association with the identified ATP synthase subunits was compromised in diabetic mitochondria, we performed analyses in control and diabetic mitochondria. Western blot analysis revealed a lighter band when measuring the association between specific ATP synthase subunits and native cardiolipin (represented by the native bound lane) in the diabetic IFM compared to control. This finding suggests that the association between the native form of cardiolipin and the ATP synthase subunits is less in the diabetic IFM compared to control. Western blot analysis also revealed a denser band when measuring the association between the subunit and cardiolipin-coated beads (represented by the bead bound lane) in the diabetic IFM compared to control. The denser band suggests that more ATP synthase subunits are not in association with cardiolipin, but are free to bind to the cardiolipincoated beads in the diabetic IFM. Taken together, these results indicate that the 
decrease in interaction between native cardiolipin and ATP synthase could be an underlying mechanism causing decreased ATP synthase activity in the diabetic IFM.

A number of studies have utilized cell lines to examine the physiological role of cardiolipin. When the structural gene encoding CRLS was disrupted resulting in depletion of cardiolipin in the mitochondrial membranes of Saccharomyces cerevisiae cells, ATPase activities, cytochrome oxidase activities, membrane potential and protein import were all decreased (18). This study concluded that cardiolipin is required for maintaining mitochondrial function. Chen et al. (5) identified and characterized the cDNA encoding human CRLS. The human CRLS was expressed in COS-7 cells from which cardiolipin was not only efficiently synthesized in vitro using CDP-DAG and PG as substrates, but was also synthesized at an increased rate. Kiebish et al. (19) determined that CRLS overexpression in a STZ-induced diabetic model attenuated mitochondrial dysfunction associated with diabetes mellitus. Although these authors did not find a difference in total cardiolipin content between diabetic and control hearts, the overexpression of CRLS increased cardiac cardiolipin remodeling and lipidomic flux, which in turn, increased tetralinoleic cardiolipin. The novel finding of CRLS-mediated regulatory control was central in the preservation of mitochondrial function during diabetic insult. This study further supports the importance of IMM preservation.

Though our data suggest that changes in essential constituents in the cardiolipin biosynthetic pathway (i.e., CRLS) are associated with a decrease in cardiolipin, it is important to note that diabetes mellitus is a multifactorial disease that influences a 
number of processes, specifically in mitochondria. Increased content of the oxidized form of cardiolipin in the diabetic IFM has been reported by our laboratory (Dabkowski et al. 2009), thus one must consider that an oxidative stress pathway may also be contributing to the results reported in this study. Increased oxidation of cardiolipin would likely predispose the phospholipid to loss, influencing mitochondrial functionality (22). Future studies in which manipulation of mitochondrial oxidative milieu specifically at the IMM may lend insight into the contribution of oxidative stress. 


\section{CONCLUSION}

In conclusion, our results indicate the importance of preserving the IMM during type 1 diabetes mellitus. We show dysregulation of the cardiolipin biosynthetic pathway during a diabetic setting, as seen by a decrease in CRLS protein content and activity in the diabetic IFM. These studies are the first to demonstrate a direct association between cardiolipin and ATP synthase $\mathrm{F}_{0}$-complex subunit $\mathrm{b}$ and $\mathrm{d}$ in both mitochondrial subpopulations. CRLS and IMM conservation represents a potential therapeutic target that may be manipulated for treatment against development of cardiomyopathy associated with the type 1 diabetic heart. 


\section{ACKNOWLEDGEMENTS}

We would like acknowledge Dr. Vazhaikkurichi Rajendran in the Biochemistry Department at West Virginia University for the assistance and expertise during the CRLS activity assay experimentation.

There are no conflicts of interests to disclose. This work was supported by the National Institutes of Health from the National Institutes of Diabetes and Digestive and Kidney Diseases [DP2DK083095]. This work was also supported by a Grant-In-Aid from the American Heart Association [0855484D]. Tara Croston is a recipient of an NIH Predoctoral Fellowship [T32HL090610]. Danielle Shepherd is a recipient of an NIH Predoctoral Fellowship [T32HL090610]. Erinne Dabkowski is a recipient of an American Heart Association Predoctoral Fellowship [0815406D]. Cody Nichols is a recipient of an Integrative Graduate Education and Research Traineeship Program [DGE-1144676]. 


\section{REFERENCES}

1. Acehan D, Malhotra A, Xu Y, Ren M, Stokes DL, and Schlame M. Cardiolipin affects the supramolecular organization of ATP synthase in mitochondria. Biophys $J$ 100: 2184-2192, 2011.

2. Acehan D, Vaz F, Houtkooper RH, James J, Moore V, Tokunaga C, Kulik W, Wansapura J, Toth MJ, Strauss A, and Khuchua Z. Cardiac and skeletal muscle defects in a mouse model of human Barth syndrome. J Biol Chem 286: 899-908, 2011.

3. Baseler WA, Dabkowski ER, Williamson CL, Croston TL, Thapa D, Powell MJ, Razunguzwa TT, and Hollander JM. Proteomic alterations of distinct mitochondrial subpopulations in the type 1 diabetic heart: contribution of protein import dysfunction. Am J Physiol Regul Integr Comp Physiol 300: R186-200, 2011.

4. Bradford MM. A rapid and sensitive method for the quantitation of microgram quantities of protein utilizing the principle of protein-dye binding. Anal Biochem 72: 248254, 1976.

5. Chen $\mathbf{D}$, Zhang $\mathbf{X Y}$, and Shi Y. Identification and functional characterization of hCLS1, a human cardiolipin synthase localized in mitochondria. Biochem $J$ 398: 169176, 2006.

6. Chicco AJ and Sparagna GC. Role of cardiolipin alterations in mitochondrial dysfunction and disease. Am J Physiol Cell Physiol 292: C33-44, 2007.

7. Dabkowski ER, Baseler WA, Williamson CL, Powell M, Razunguzwa TT, Frisbee JC, and Hollander JM. Mitochondrial dysfunction in the type 2 diabetic heart is associated with alterations in spatially distinct mitochondrial proteomes. Am J Physiol Heart Circ Physiol 299: H529-540, 2010. 
8. Dabkowski ER, Williamson CL, Bukowski VC, Chapman RS, Leonard SS, Peer CJ, Callery PS, and Hollander JM. Diabetic cardiomyopathy-associated dysfunction in spatially distinct mitochondrial subpopulations. Am J Physiol Heart Circ Physiol 296: H359-369, 2009.

9. Dabkowski ER, Williamson CL, and Hollander JM. Mitochondria-specific transgenic overexpression of phospholipid hydroperoxide glutathione peroxidase (GPx4) attenuates ischemia/reperfusion-associated cardiac dysfunction. Free Radic Biol Med 45: 855-865, 2008.

10. Devereux RB, Roman MJ, Paranicas M, OÂ'Grady MJ, Lee ET, Welty TK, Fabsitz RR, Robbins D, Rhoades ER, and Howard BV. Impact of Diabetes on Cardiac Structure and Function : The Strong Heart Study. Circulation 101: 2271-2276, 2000.

11. Eble KS, Coleman WB, Hantgan RR, and Cunningham CC. Tightly associated cardiolipin in the bovine heart mitochondrial ATP synthase as analyzed by 31P nuclear magnetic resonance spectroscopy. J Biol Chem 265: 19434-19440, 1990.

12. Fry M and Green DE. Cardiolipin requirement for electron transfer in complex I and III of the mitochondrial respiratory chain. Journal of Biological Chemistry 256: 18741880, 1981.

13. Haines TH and Dencher NA. Cardiolipin: a proton trap for oxidative phosphorylation. FEBS Letters 528: 35-39, 2002.

14. Han X, Yang J, Cheng H, Yang K, Abendschein DR, and Gross RW. Shotgun lipidomics identifies cardiolipin depletion in diabetic myocardium linking altered 
substrate utilization with mitochondrial dysfunction. Biochemistry 44: 16684-16694, 2005.

15. Han X, Yang J, Yang K, Zhao Z, Abendschein DR, and Gross RW. Alterations in Myocardial Cardiolipin Content and Composition Occur at the Very Earliest Stages of Diabetes: A Shotgun Lipidomics Study. Biochemistry 46: 6417-6428, 2007.

16. Hatch GM, Cao SG, and Angel A. Decrease in cardiac phosphatidylglycerol in streptozotocin-induced diabetic rats does not affect cardiolipin biosynthesis: evidence for distinct pools of phosphatidylglycerol in the heart. Biochem J 306 ( Pt 3): 759-764, 1995.

17. Houtkooper R and Vaz F. Cardiolipin, the heart of mitochondrial metabolism. Cellular and Molecular Life Sciences 65: 2493-2506, 2008.

18. Jiang F, Ryan MT, Schlame M, Zhao M, Gu Z, Klingenberg M, Pfanner N, and Greenberg ML. Absence of cardiolipin in the crd1 null mutant results in decreased mitochondrial membrane potential and reduced mitochondrial function. $J$ Biol Chem 275: 22387-22394, 2000.

19. Kiebish MA, Yang K, Sims HF, Jenkins CM, Liu X, Mancuso DJ, Zhao Z, Guan S, Abendschein DR, Han X, and Gross RW. Myocardial Regulation of Lipidomic Flux by Cardiolipin Synthase: SETTING THE BEAT FOR BIOENERGETIC EFFICIENCY. J Biol Chem 287: 25086-25097, 2012.

20. Laemmli UK. Cleavage of structural proteins during the assembly of the head of bacteriophage T4. Nature 227: 680-685, 1970. 
21. Lu B, Xu FY, Jiang YJ, Choy PC, Hatch GM, Grunfeld C, and Feingold KR. Cloning and characterization of a cDNA encoding human cardiolipin synthase (hCLS1). J Lipid Res 47: 1140-1145, 2006.

22. Ma ZA, Zhao Z, and Turk J. Mitochondrial dysfunction and beta-cell failure in type 2 diabetes mellitus. Exp Diabetes Res 2012: 703538, 2012.

23. Minkler PE and Hoppel CL. Separation and characterization of cardiolipin molecular species by reverse-phase ion pair high-performance liquid chromatographymass spectrometry. Journal of Lipid Research 51: 856-865, 2010.

24. Nie J, Hao X, Chen D, Han X, Chang Z, and Shi Y. A novel function of the human CLS1 in phosphatidylglycerol synthesis and remodeling. Biochim Biophys Acta 1801: 438-445, 2010.

25. Palmer JW, Tandler B, and Hoppel CL. Biochemical properties of subsarcolemmal and interfibrillar mitochondria isolated from rat cardiac muscle. Journal of Biological Chemistry 252: 8731-8739, 1977.

26. Schlame M, Rua D, and Greenberg ML. The biosynthesis and functional role of cardiolipin. Prog Lipid Res 39: 257-288, 2000.

27. Sedlak E and Robinson NC. Phospholipase A(2) digestion of cardiolipin bound to bovine cytochrome c oxidase alters both activity and quaternary structure. Biochemistry 38: 14966-14972, 1999.

28. Severson DL. Diabetic cardiomyopathy: recent evidence from mouse models of type 1 and type 2 diabetes. Can J Physiol Pharmacol 82: 813-823, 2004.

29. Sparagna GC, Johnson CA, McCune SA, Moore RL, and Murphy RC. Quantitation of cardiolipin molecular species in spontaneously hypertensive heart failure 
rats using electrospray ionization mass spectrometry. Journal of Lipid Research 46: 1196-1204, 2005.

30. Taylor WA, Xu FY, Ma BJ, Mutter TC, Dolinsky VW, and Hatch GM. Expression of monolysocardiolipin acyltransferase activity is regulated in concert with the level of cardiolipin and cardiolipin biosynthesis in the mammalian heart. $B M C$ Biochem 3: 9, 2002.

31. Tomita M, Mukae S, Geshi E, Umetsu K, Nakatani M, and Katagiri T. Mitochondrial respiratory impairment in streptozotocin-induced diabetic rat heart. Jpn Circ J 60: 673-682, 1996.

32. Waring AJ, Rottenberg H, Ohnishi T, and Rubin E. Membranes and phospholipids of liver mitochondria from chronic alcoholic rats are resistant to membrane disordering by alcohol. Proc Natl Acad Sci U S A 78: 2582-2586, 1981.

33. Williamson CL, Dabkowski ER, Baseler WA, Croston TL, Alway SE, and Hollander JM. Enhanced apoptotic propensity in diabetic cardiac mitochondria: influence of subcellular spatial location. Am J Physiol Heart Circ Physiol 298: H633-642, 2010.

34. Wu KK and Huan Y. Streptozotocin-Induced Diabetic Models in Mice and Rats. In: Current Protocols in Pharmacology: John Wiley \& Sons, Inc., 2001. 
Figure 2.1

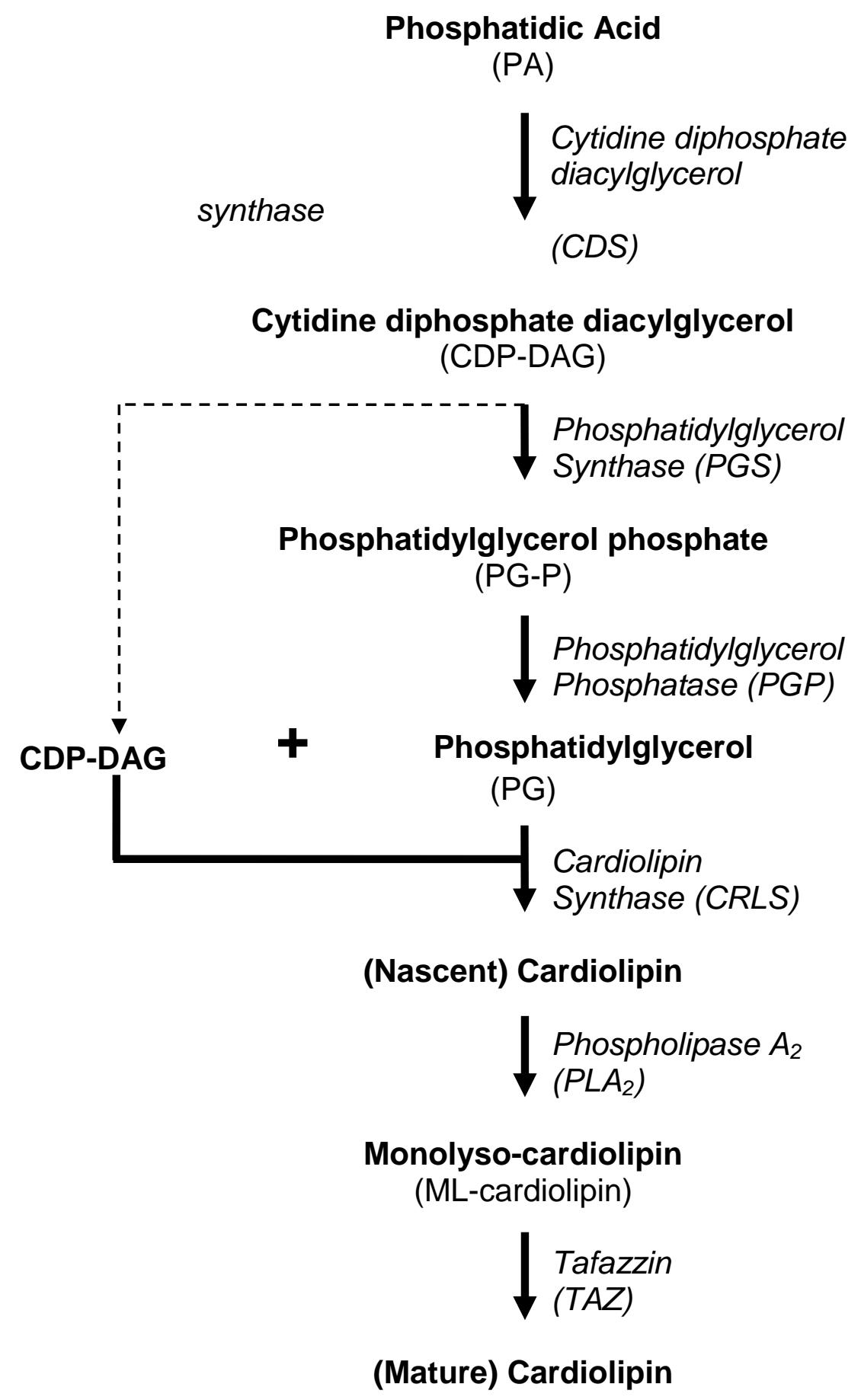


Figure 2.1 Cardiolipin biosynthetic pathway. Schematic diagram illustrating cardiolipin biosynthetic pathway. 
Figure 2.2

A.

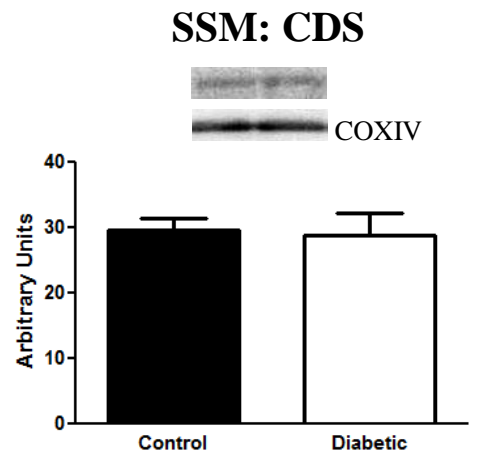

C.

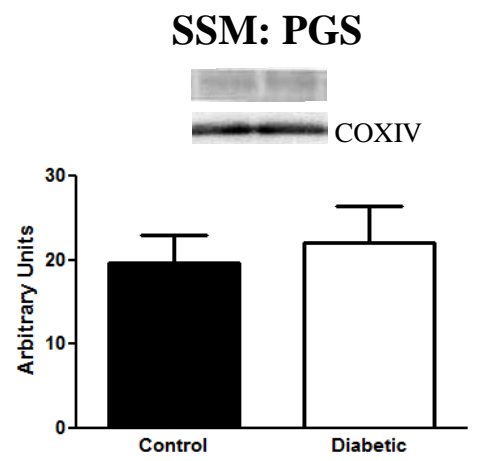

E.

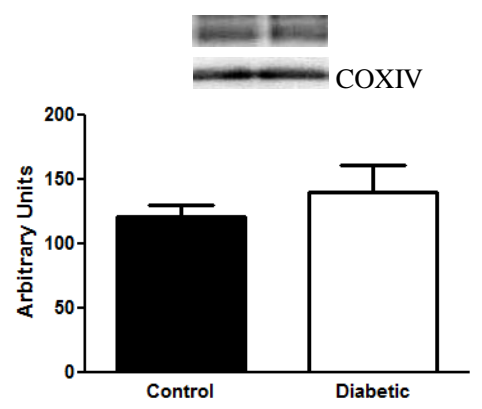

G.

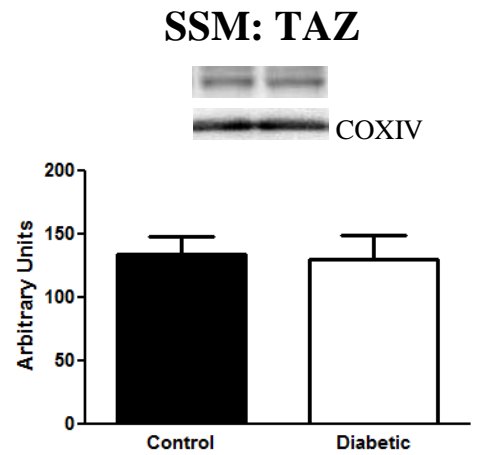

B.

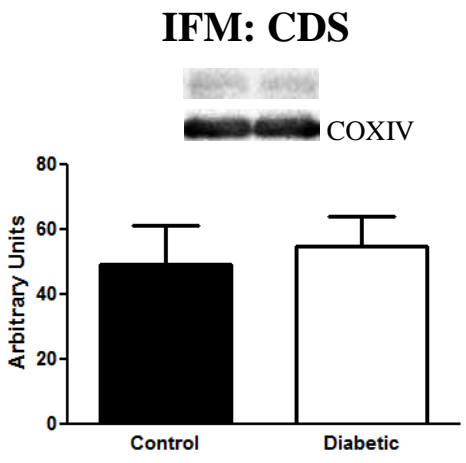

D.

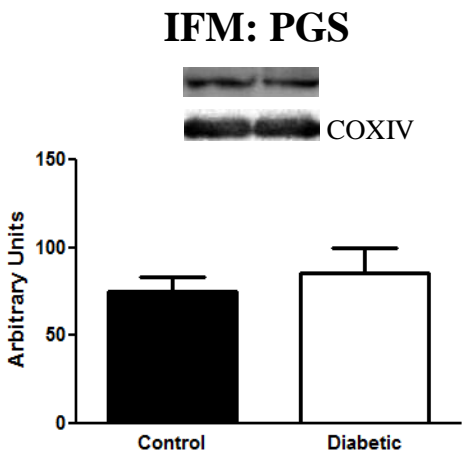

F.

IFM: CRLS

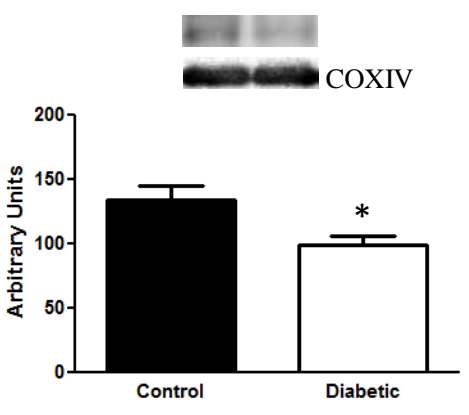

H.

IFM: TAZ

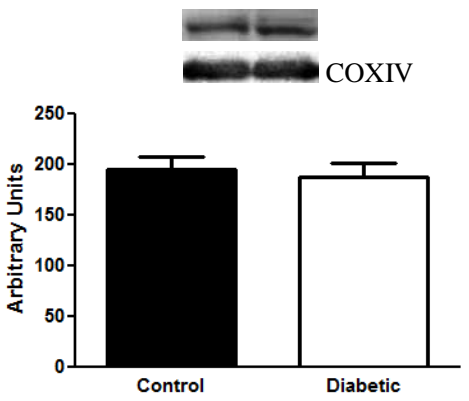


Figure 2.2 Protein content of cardiolipin biosynthetic pathway constituents. Assessment of cardiolipin biosynthetic pathway constituents was performed by Western blot using specific antibodies. (A) and (B): representative CDS content from SSM and IFM control $(n=7)$ and diabetic $(n=8)$, respectively; (C) and (D): representative PGS content from SSM ( $\mathrm{n}=7$ for each group) and IFM ( $\mathrm{n}=8$ for each group) control and diabetic, respectively; (E) and (F): representative CRLS content from SSM and IFM control $(n=8)$ and diabetic $(n=7)$, respectively; $(\mathbf{G})$ and $(\mathbf{H})$ : representative TAZ content from SSM and IFM control $(n=7)$ and diabetic $(n=7)$, respectively. Control for loading was confirmed using COX IV probing. Values are expressed as means \pm SEM; ${ }^{*} P<0.05$ for control vs. diabetic groups. Abbreviations: $C D S=$ cytidine diphosphate diacylglycerol synthase $;$ PGS = phosphatidylglycerol synthase $;$ CRLS = cardiolipin synthase $;$ TAZ = tafazzin; IFM = interfibrillar mitochondria; SSM = subsarcolemmal mitochondria. 
Figure 2.3

A.

SSM

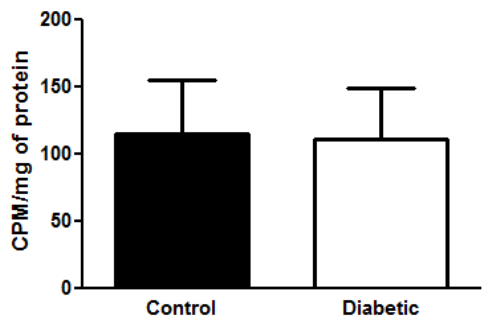

c.

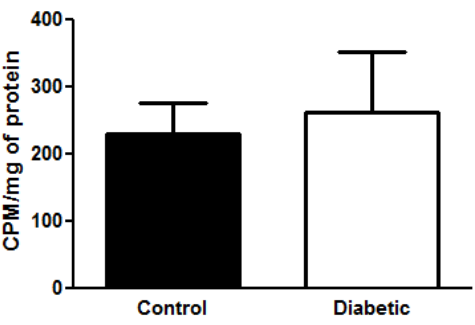

B.

IFM

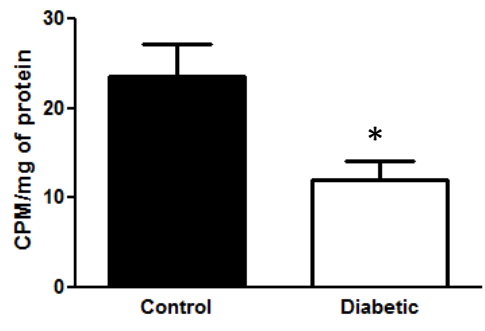

IFM

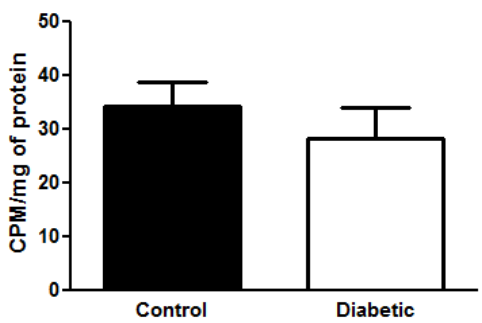


Figure 2.3 Cardiolipin synthase (CRLS) activity. CRLS activity was assessed using a ${ }^{14} \mathrm{C}$-acyl donor. (A): CRLS activity measured in SSM diabetic and control samples. (B): CRLS activity measured in IFM diabetic samples compared to control samples. (C): PG radioactive counts/minute/mg of protein measured in SSM diabetic samples compared to control samples. (D): PG radioactive counts/minute/mg of protein measured in IFM diabetic samples compared to control samples. Values are expressed as means $\underline{\mathbf{S}} \mathrm{SEM}$; $\mathrm{n}=8$ for each group. ${ }^{*} P<0.05$ for control vs. diabetic groups. Abbreviations: $\mathrm{CDS}=$ cytidine diphosphate diacylglycerol synthase; $\mathrm{PG}=$ phosphatidylglycerol; $\mathrm{IFM}=$ interfibrillar mitochondria; SSM = subsarcolemmal mitochondria. 
Figure 2.4

A.

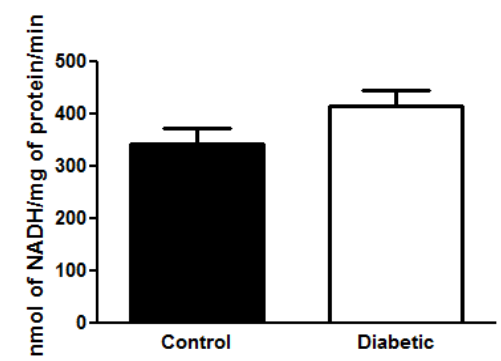

B.

IFM

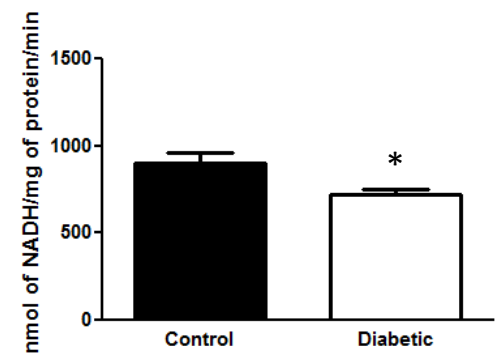


Figure 2.4 ATP synthase activity. Spectrophotometric analysis of ATP synthase activities in control and diabetic samples. (A): ATP synthase activity from SSM control and diabetic groups. (B): ATP synthase activity from IFM control and diabetic groups. Values are expressed as means $\pm \mathrm{SEM} ; \mathrm{n}=4$ for each group. ${ }^{*} P<0.05$ for control vs. diabetic groups. Abbreviations: IFM = interfibrillar mitochondria; SSM = subsarcolemmal mitochondria. 
Figure 2.5

A.

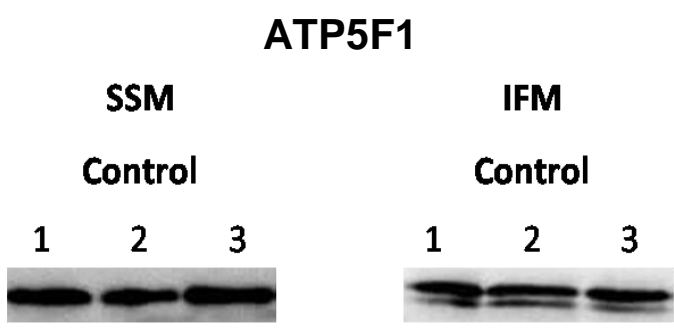

c.

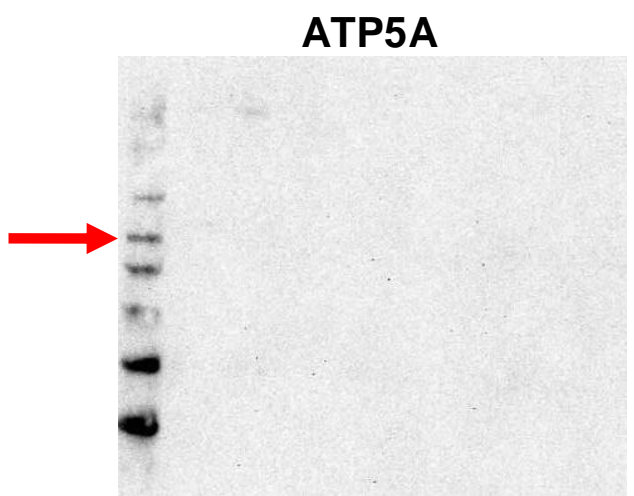

E.

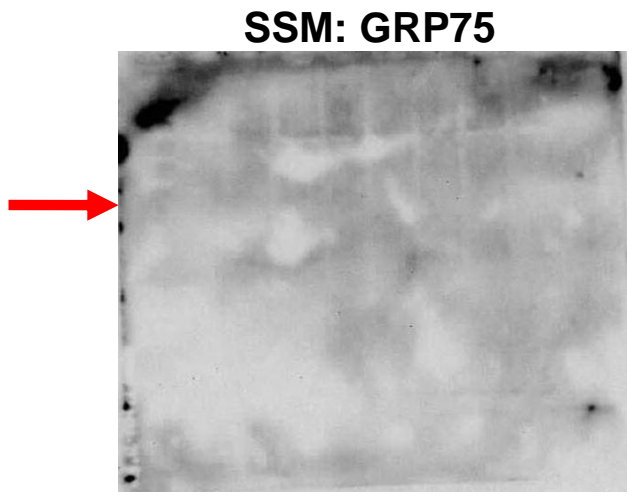

B.

\begin{tabular}{cccccc} 
& \multicolumn{3}{c}{ ATP5H } & \multicolumn{2}{c}{ IFM } \\
& & \multicolumn{2}{c}{$\begin{array}{c}\text { IFM } \\
\text { Control }\end{array}$} & & \multicolumn{2}{c}{ Control } \\
1 & 2 & 3 & 1 & 2 & 3 \\
\hline
\end{tabular}

D.

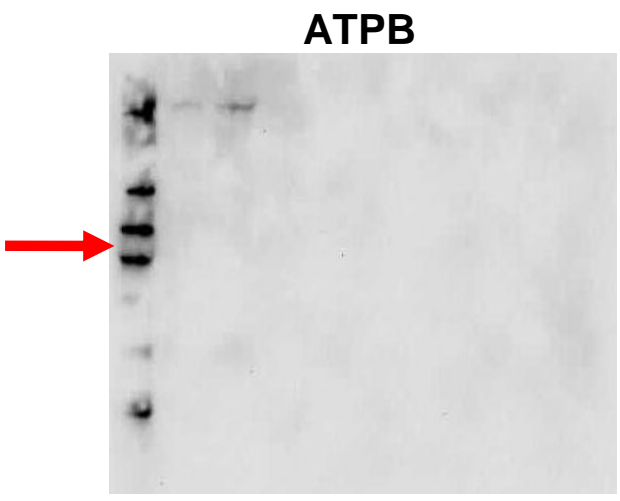

F.

IFM: GRP75

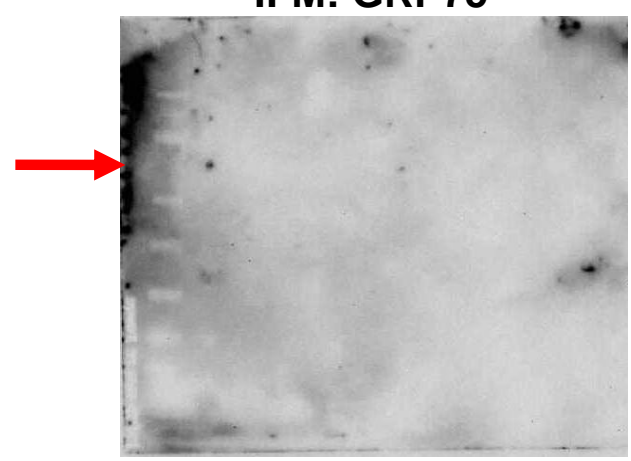


Figure 2.5 Cardiolipin - ATP synthase association. Mitochondrial protein was incubated with cardiolipin-coated beads. Western blots were performed to determine associations between cardiolipin and (A): ATP synthase subunit b (ATP5F1); $n=3$ for each group and (B): ATP synthase subunit $d(A T P H) ; n=3$ for each group (C): ATP synthase alpha (ATP5A); $n=2$ for each group and (D): ATP synthase beta (ATPB); $n=2$ for each group and (E): Glucose regulated protein (GRP75) in the SSM; $n=3$ for each group and (F): Glucose regulated protein (GRP75) in the IFM; $n=3$ for each group using specific antibodies (Representative blots shown). Abbreviations: IFM = interfibrillar mitochondria; SSM = subsarcolemmal mitochondria. 
Figure 2.6

A.

SSM

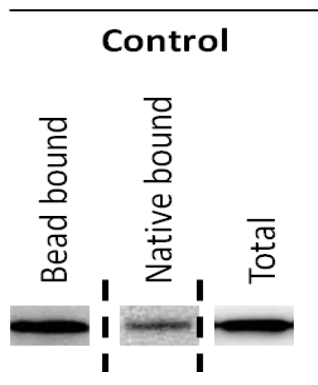

B.

SSM

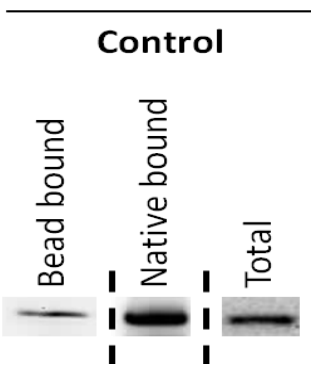

Diabetic

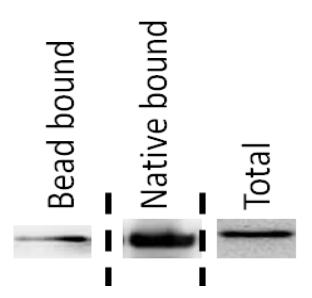

C.

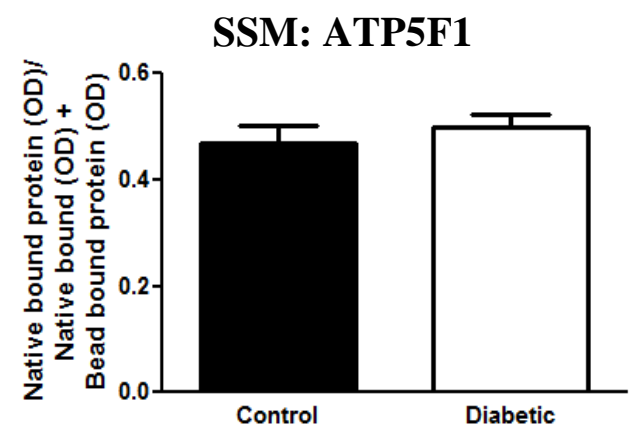

E.

IFM: ATP5F1

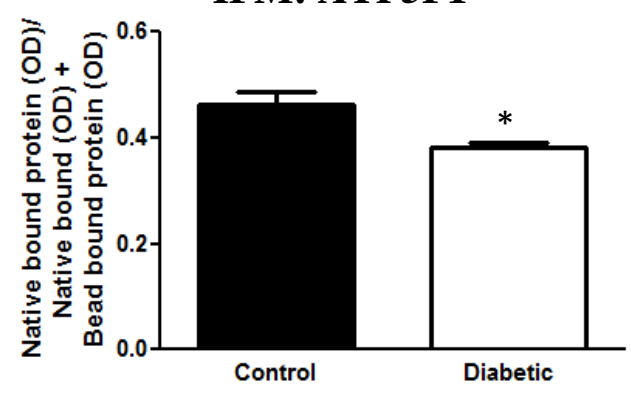

ATP5F1

IFM

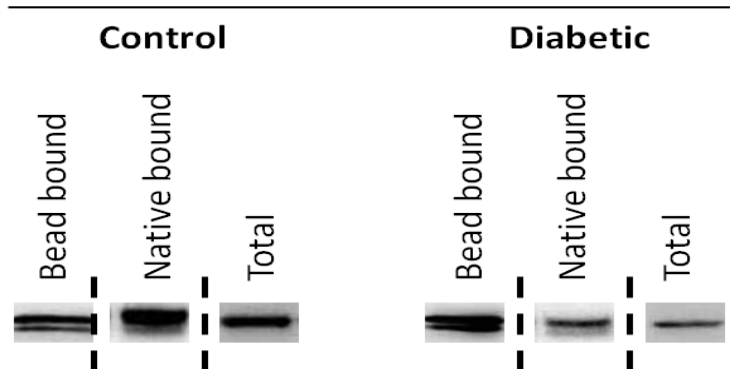

ATP5H

IFM

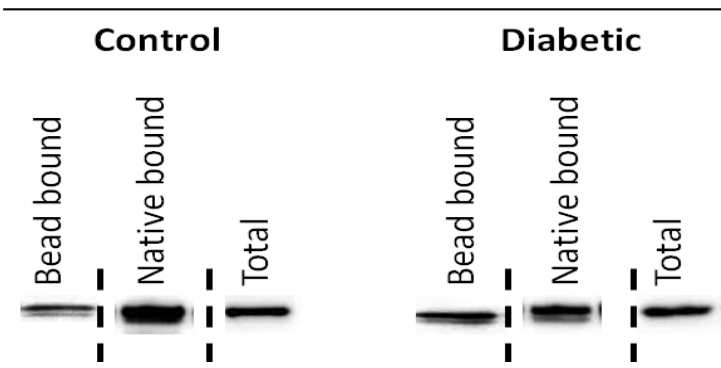

D. SSM: ATP5H

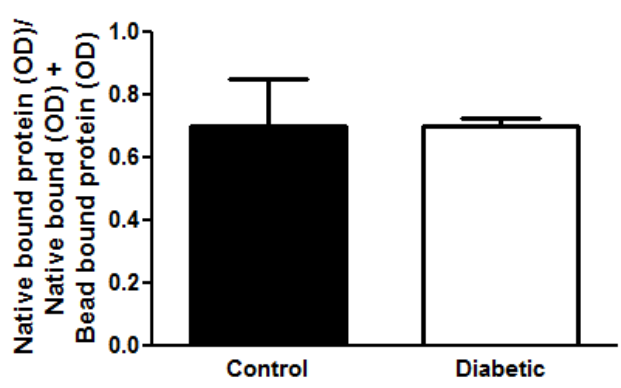

F. IFM: ATP5H

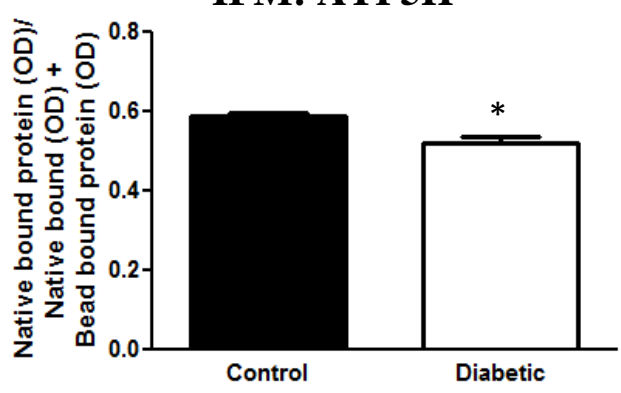


Figure 2.6 Native cardiolipin - ATP synthase association. The association between cardiolipin and the detected interactions were measured in control and diabetic mitochondrial subpopulations. (A): Representation of ATP5F1 protein content in control and diabetic SSM and IFM, respectively. The "Bead bound" column represents ATP5F1 that was eluted off of the cardiolipin-coated beads, whereas the "Native bound" column represents ATP5F1 associated with native cardiolipin that was present in the supernatant. The "Total" band represents the total ATP5F1 protein content in isolated mitochondria. (B): Representation of ATP5H in the same manner as ATP5F1 (Figure 2.6A). Quantification of the association between native cardiolipin and ATP5F1 in control and diabetic hearts in (C): SSM and (E): IFM. Quantification of the association between native cardiolipin and ATP5H in control and diabetic hearts in (D): SSM and (F): IFM. Values are expressed as means \pm SEM; $\mathrm{n}=3$ for each group. ${ }^{*} P<0.05$ for control vs. diabetic groups. Abbreviations: IFM = interfibrillar mitochondria; SSM = subsarcolemmal mitochondria. 
Table 2.1

\begin{tabular}{|c|c|c|}
\hline mRNA & \begin{tabular}{|c|}
$\begin{array}{c}\text { Diabetic vs. } \\
\text { Control }\end{array}$ \\
\end{tabular} & p-value \\
\hline \multicolumn{3}{|l|}{ Cardiolipin biosynthesis } \\
\hline Cytidine diphosphate-diacylglycerol synthase 1 & 1.18 & 0.17 \\
\hline Phosphatidylglycerol synthase 1 & 0.92 & 0.47 \\
\hline Cardiolipin synthase & 0.98 & 0.91 \\
\hline Tafazzin & 0.86 & 0.24 \\
\hline \multicolumn{3}{|l|}{ Protein import } \\
\hline Mitochondrial import inner membrane translocase subunit TIM16 & 0.98 & 0.89 \\
\hline Mitochondrial import inner membrane translocase subunit TIM14 & 0.97 & 0.98 \\
\hline \multicolumn{3}{|l|}{ Oxidative phosphorylation } \\
\hline NADH dehydrogenase [ubiquinone] flavoprotein 1 , mitochondrial & 0.91 & 0.20 \\
\hline NADH dehydrogenase [ubiquinone] flavoprotein 2 , mitochondrial & 1.05 & 0.60 \\
\hline NADH dehydrogenase [ubiquinone] 1 alpha subcomplex subunit 1 & 0.97 & 0.84 \\
\hline NADH dehydrogenase [ubiquinone] 1 alpha subcomplex subunit 2 & 0.98 & 0.92 \\
\hline NADH dehydrogenase [ubiquinone] 1 alpha subcomplex subunit 3 & 0.94 & 0.45 \\
\hline NADH dehydrogenase [ubiquinone] 1 alpha subcomplex subunit 4 & 0.95 & 0.63 \\
\hline NADH dehydrogenase [ubiquinone] 1 alpha subcomplex subunit 12 & 0.92 & 0.62 \\
\hline Succinate dehydrogenase [ubiquinone] iron-sulfur subunit, mitochondrial & 1.02 & 0.88 \\
\hline Electron transfer flavoprotein-ubiquinone oxidoreductase, mitochondrial & 0.62 & 0.000049 \\
\hline Cytochrome b-c1 complex subunit 1 , mitochondrial & 1.06 & 0.49 \\
\hline Cytochrome b-c1 complex subunit Rieske, mitochondrial & 0.96 & 0.69 \\
\hline Cytochrome $\mathrm{c} 1$, heme protein, mitochondrial & 0.99 & 0.98 \\
\hline ATP synthase subunit $b$, mitochondrial & 0.97 & 0.75 \\
\hline \multicolumn{3}{|l|}{ Miscellaneous } \\
\hline Prohibitin-2 & 0.88 & 0.40 \\
\hline
\end{tabular}


Table 2.1. mRNA analysis of inner mitochondrial membrane proteins. mRNA was isolated from control and diabetic hearts. The mRNA content levels for inner mitochondrial membrane proteins were assessed. Values are expressed as fold change in diabetic heart as compared to control heart; $n=4$ for each group. The bold value indicates a $p$-Value $<0.05$ for diabetic vs. control hearts. 


\section{Chapter 3:}

\section{Mitochondrial Dysfunction in the Human Diabetic Heart: Impact on Distinct Mitochondrial Subpopulations}

As submitted in AJP Heart and Circulatory Physiology, 2013 August

Tara L. Croston ${ }^{1}$, Anthony A. Holden ${ }^{2}$, Kevin Tveter $^{2}$, Sara E. Lewis ${ }^{1}$, Dharendra Thapa $^{1}$, Danielle L. Shepherd ${ }^{1}$, Cody E. Nichols ${ }^{1}$, Rajaganapathi Jagannathan ${ }^{1}$, Dustin M. Long ${ }^{3}$, I. Mark Olfert ${ }^{1}$, John M. Hollander ${ }^{1}$

${ }^{1}$ West Virginia University School of Medicine, Division of Exercise Physiology and Center for Cardiovascular and Respiratory Sciences, Morgantown, WV 26506

${ }^{2}$ West Virginia University School of Medicine, Department of Surgery, Morgantown, WV 26506

${ }^{3}$ West Virginia University School of Public Health, Department of Biostatistics, Morgantown, WV 26506

Running Title: Mitochondrial dysfunction in the diabetic heart 


\section{ABSTRACT}

Mitochondrial dysfunction is a central contributor to the development of diabetic cardiomyopathy. The examination of mitochondrial function is further complicated by the existence of two cardiac mitochondrial subpopulations, subsarcolemmal mitochondria (SSM) and interfibrillar (IFM) mitochondria. We have previously reported decreased SSM function in type $2 \mathrm{db} / \mathrm{db}$ mouse heart. Currently, subpopulation based mitochondrial analyses have not been explored in type 2 diabetic human heart. The goal of this study was to examine the impact of type 2 diabetic insult on mitochondrial function in the human heart. Mitochondrial subpopulations were isolated from atrial appendages of non-diabetic and diagnosed type 2 diabetic patients. Glucose-mediated and fatty acid-mediated state 3 and state 4 respiration rates were decreased in diabetic SSM as compared to non-diabetic SSM ( $P \leq 0.05$ for both), with no change in IFM. Electron transport chain complexes I and IV activities were decreased in diabetic SSM compared to non-diabetic SSM ( $P \leq 0.05$ for both), with a concomitant decrease in complex I and IV protein content in diabetic SSM ( $P \leq 0.05$ for both). The primary factor accounting for mitochondrial function was diabetes mellitus, when compared to other co-morbidities such as coronary artery disease and hypertension. Using spline models to determine correlative risk for mitochondrial dysfunction indicated that diabetic patients display the same level of state 3 and electron transport chain complex I dysfunction regardless of absolute glycated hemoglobin (HbA1c) and blood glucose

levels. Overall, the results suggest that independent of other pathologies mitochondrial dysfunction is present in the SSM of type 2 diabetic patients and identifies a threshold 
where mitochondrial dysfunction is present regardless of absolute HbA1c or blood glucose levels. 


\section{INTRODUCTION}

Diabetes mellitus is a condition that is increasingly becoming an epidemic, with an estimated 330 million affected worldwide by the year 2030 (10). Of the individuals diagnosed with the disease, type 2 diabetes mellitus accounts for approximately 90$95 \%$ of cases (10), and has been attributed to sedentary lifestyle (20). Type 2 diabetes mellitus results from insulin resistance caused by an imbalance of the body's glucose homeostasis (20). The body does not properly use the insulin produced in response to increased blood glucose levels; therefore, the pancreas eventually loses its ability to produce insulin (10).

Cardiovascular complications are the leading cause of morbidity and mortality in individuals with diabetes mellitus (17). Mitochondrial dysfunction has been shown to contribute to the development of these cardiovascular complications during type 2 diabetes mellitus $(7,8,13)$. In the cardiomyocyte, there are two mitochondrial subpopulations that are morphologically, biochemically, and spatially distinct $(15,23)$.

The two separate pools of mitochondria respond differently in the face of a cardiovascular insult, such as diabetes, aging, and ischemia/reperfusion $(13,14,21$, 22). The subsarcolemmal mitochondria (SSM) are located just beneath the sarcolemma, whereas the interfibrillar mitochondria (IFM) are located in between the myofibrils. 
Previously, examination in a type 2 diabetic $\mathrm{db} / \mathrm{db}$ mouse model revealed cardiac dysfunction $(6,28)$. When mitochondrial function was assessed, decreased respiration rates and oxidative phosphorylation were observed (26). Upon examination of the two separate pools of mitochondria, our laboratory observed decreased respiration and oxidative phosphorylation, as well as an altered proteomic profile primarily in the diabetic SSM, with the IFM being affected to a lesser extent (13).

Though animal studies have provided convincing evidence of differential responses by spatially-distinct mitochondrial subpopulations during type 2 diabetic insult, studies confirming these effects in a human patient population are limited. Skeletal muscle biopsies have been revealed decreased mitochondrial function in skeletal muscle of type 2 diabetic patients (19), specifically in the SSM subpopulation (25). Fewer studies have examined the human heart due to the invasive procedure required to obtain human heart tissue. Anderson et al. reported decreased mitochondrial function in type 2 diabetic atria $(3,4)$. Nevertheless, it is still unclear as to how diabetes mellitus affects mitochondrial subpopulations in the diabetic human heart.

The goal of this study was to determine how mitochondrial subpopulations are affected in the heart of type 2 diabetic patients compared to non-diabetic patients. Our results suggest that type 2 diabetes mellitus negatively impacts the SSM to a greater extent than the IFM and that the observed dysfunction may be attributed to decreased oxidative phosphorylation machinery. Our data also demonstrate that compounding comorbidities, such as coronary artery disease (CAD), hypertension and body mass index 
(BMI), alone do not influence the observed mitochondrial dysfunction in the type 2 diabetic SSM. 


\section{MATERIALS AND METHODS}

\section{Human Patient Population}

The West Virginia University Institutional Review Board and Institutional Biosafety Committee approved all protocols. Individuals undergoing coronary artery bypass graft surgery or cardiac valve replacement at Ruby Memorial Hospital consented for the release of their cardiac tissue to West Virginia University School of Medicine. Consenting individuals were then characterized as non-diabetic or diabetic based upon prior diagnosis of diabetes mellitus. All demographic and clinical data are shown in Table 1.

\section{Individual Mitochondrial Subpopulations Isolation}

Right atrial appendages were removed from patients and rinsed in phosphate buffered saline (PBS, $\mathrm{pH}$ 7.4). Pericardial fat was then trimmed and the heart tissue was weighed. The SSM and IFM were isolated as previously described by Palmer et al. (23) with modifications by our laboratory $(15,16,33)$. Mitochondrial pellets were resuspended in KME buffer (100 $\mathrm{mM} \mathrm{KCl}, 50 \mathrm{mM}$ MOPS, and $0.5 \mathrm{mM}$ EDTA) for

mitochondrial respiration, flow cytometric analyses, and enzymatic activity measurements. Protein content was determined by the Bradford method using bovine serum albumin as a standard (9). 


\section{Mitochondrial Size, Internal Complexity and Membrane Potential}

Mitochondrial subpopulation size, internal complexity and mitochondrial membrane potential were examined as previously described by our laboratory with modifications $(13,15,33)$. Briefly, flow cytometric analyses were performed using a FACS Calibur equipped with a 15-MW 488-nm argon laser and 633-nm red diode laser (Becton Dickinson, San Jose, CA). The ratiometric dye 5,5',6,6'-tetrachloro-1,1',3,3'tetraethylbenzimidazol carbocyanine iodide (JC-1; Molecular Probes, Carlsbad, CA), was used to selectively stain and gate for respiring mitochondria. Freshly isolated mitochondrial subpopulations were incubated with $\mathrm{JC}-1$ for 15 min at $37^{\circ} \mathrm{C}$, and the samples were run for 30 seconds. Forward scatter (FSC) and side scatter (SSC) detectors were used to examine size (FSC) and complexity (SSC) in isolated mitochondrial subpopulations. Changes in membrane potential were recorded as the ratio between the color change from green to orange. Measurements were performed on freshly isolated mitochondrial subpopulations. All flow cytometric measurements were performed in conjunction with the West Virginia University Flow Cytometry Core Facility.

\section{Mitochondrial Respiration Rates}

State 3 and state 4 respiration rates were measured in isolated mitochondrial subpopulations as previously described $(11,12,18)$, with modifications (16). Briefly, isolated mitochondrial subpopulations were resuspended in KME buffer. Protein content was determined by the Bradford method (9). Fifty micrograms of protein was added to respiration buffer $(80 \mathrm{mmol} / \mathrm{K} \mathrm{KCl}, 50 \mathrm{mmol} / \mathrm{l} \mathrm{MOPS}, 1 \mathrm{mmol} / \mathrm{l} \mathrm{EGTA}, 5 \mathrm{mmol} / \mathrm{K} \mathrm{KH} 2 \mathrm{PO} 4$, 
and $1 \mathrm{mg} / \mathrm{ml} \mathrm{BSA}$ ) and placed into the respiration chamber connected to an oxygen probe (OX1LP-1mL Dissolved Oxygen Package, Qubit System, Kingston, ON, Canada). Maximal glucose-mediated respiration was initiated by the addition of glutamate ( $5 \mathrm{mM})$ and malate $(5 \mathrm{mM})$. Maximal fatty acid-mediated respiration was initiated by the addition of palmitoylcarnitine $(40 \mu \mathrm{M})$ and malate $(5 \mathrm{mM})$. Measurements of state $3(250 \mathrm{mM}$ ADP) and state 4 (ADP-limited) respiration were made as previously described (7). Values were expressed as nmol of oxygen consumed $-\mathrm{min}^{-1}$-mg protein ${ }^{-1}$.

\section{Electron Transport Chain (ETC) Complex Activities}

Complex I, complex III, and complex IV activities were measured spectrophotometrically as previously described $(13,15,16,29)$. Complex I activity was determined by measuring the oxidation of NADH at $340 \mathrm{~nm}$ and complex III activity was determined by measuring the reduction of cytochrome $c$ at $550 \mathrm{~nm}$ in the presence of reduced decylubiquinone $(50 \mu \mathrm{M})$. Complex IV activity was determined by measuring the oxidation of cytochrome $c$ at $550 \mathrm{~nm}$. Protein content was determined by the Bradford method (9), and values were expressed as activities (in nmol substrate consumed $\left.\cdot \mathrm{min}^{-1} \cdot \mathrm{mg} \mathrm{protein}^{-1}\right)$.

\section{Electron Transport Chain Complex Protein Expression}

To assess electron transport chain complex I and IV abundance within the nondiabetic and diabetic human heart, blue native polyacrylamide gel electrophoresis (BNPAGE) was performed according to the manufacturer's protocol (Invitrogen, Carlsbad, CA). Briefly, $20 \mu \mathrm{g}$ of isolated mitochondria were solubilized with $1 \%$ digitonin for 15 min 
on ice. After addition of $1.25 \mu$ of Coomassie G-250, samples were run on $4-16 \%$ NativePAGE gels at $120 \mathrm{~V}$ for $90 \mathrm{~min}$ at room temperature. Gels were transferred to PDVF membranes and subsequently probed with an anti-ND-1 goat antibody (Product No. 20493, Santa Cruz, CA) and an anti-cytochrome $c$ oxidase rabbit antibody (product No. ab16056, Abcam, Cambridge, MA). The secondary antibodies used were a donkey anti-goat IgG horseradish peroxidase conjugate (Product No. 2020, Santa Cruz, CA) and a goat anti-rabbit IgG horseradish peroxidase conjugate (product No. 10004301, Cayman Chemical Company, Ann Arbor, MI). Detection of signal was performed according to the Pierce ECL Western Blotting Substrate detection system manufacturer's directions (Thermo Fisher Scientific, Rockford, IL). Quantification of chemiluminescent signals were assessed using a G:Box Bioimaging System (Syngene, Frederick, MD), and the data captured using GeneSnap software (Syngene, Frederick, MD). Densitometry was measured using Image J Software (National Institutes of Health, Bethesda, MD).

\section{Statistics}

Means and SEM were calculated for all data sets. Data shown in Table 1 and in all subsequent figures were analyzed with a Student's $t$-test. $P<0.05$ was considered statistically significant. Logistic regression was performed to determine if any comorbities were associated with being diabetic. For patients with HbA1c measurements, the relationship between $\mathrm{HbA} 1 \mathrm{c}$ and the outcomes were assessed using a linear spline model with a break-point at $\mathrm{HbA1c}$ equal to $6.5 \%$. The World Health Organization states that a patient is considered diabetic if $\mathrm{HbA1c}$ level is equal to or greater than $6.5 \%$ (32). 
This allowed for different linear relationships for those with HbA1c less than $6.5 \%$ and those above $6.5 \%$. The relationship between blood glucose levels and the outcomes were also assessed using a linear spline model with a break-point at a blood glucose level equal to $200 \mathrm{mg} / \mathrm{dL}$, which is the level used by the American Diabetes Association to diagnose a patient as diabetic (1). 


\section{RESULTS}

\section{Patient Characteristics Table}

As seen in Table 3.1, eighty one patients were separated into two groups, nondiabetics and diabetics, based on prior diagnosis. The ages and sexes between the two groups were similarly distributed and all the patients were Caucasian. The diabetic patients had significantly higher BMI levels, higher HbA1c levels, as well as higher

blood glucose levels ( $P \leq 0.05$ for all three). Greater than $80 \%$ of both populations had hypertension and CAD. Medications that the patients were receiving were also noted. Diabetic patients were actively receiving medications, such as Metformin or insulin, as treatment for diabetes mellitus. The majority of patients regardless of diabetic status were receiving beta-blockers, angiotensin-converting-enzyme (ACE) inhibitors and/or 3hydroxy-3-methyl-glutaryl-CoA reductase inhibitors (statins).

\section{Mitochondrial Morphology and Membrane Potential}

Literature has suggested that mitochondrial dysfunction contributes to mitochondrial morphological alterations $(13,19,25)$. Isolated mitochondria were analyzed by flow cytometry using a dye that only migrates into respiring mitochondria that contain a membrane potential. The mitochondria that were respiring were gated, which allowed only those mitochondria, and not debris, to be measured. Forward and side scatter histograms revealed two different populations, as determined by size and complexity (Figure 3.1A and 3.1B, respectively). No differences in mitochondrial size or internal complexity were observed between diabetic and non-diabetic patients for either 
subpopulation (Figures 3.1C and 3.1D). Although we did not see an alteration in mitochondrial size and internal complexity due to type 2 diabetes mellitus, we observed that the SSM were larger in size and had a greater internal complexity compared to IFM ( $P \leq 0.05$; Figure 3.1C and 3.1D, respectively), which is supported by previous findings in mouse models $(13,15,33)$.

Using a mouse model, our laboratory has previously reported higher membrane potential in the IFM compared to the SSM measured by flow cytometry $(5,15)$. In the human heart, IFM exhibited a greater membrane potential compared to SSM but no change was observed between non-diabetic and diabetic patients $(P \leq 0.05$; Figure 3.1E).

\section{Mitochondrial Respiration}

Mitochondrial respiration rates were measured in isolated mitochondrial subpopulations from non-diabetic and diabetic patients. When using glutamate and malate as substrates to measure maximal glucose-mediated respiration, diabetic SSM displayed significantly decreased state 3 and state 4 respiration rates as compared to the non-diabetic SSM $(P \leq 0.05$; Figure 3.2A), with no differences observed in the IFM (Figure 3.2B).

Glycolysis is impaired during type 2 diabetes mellitus; therefore, the heart must rely primarily on fatty acid oxidation as an energy source (2). When using palmitoylcarnitine and malate as substrates to measure maximal fatty-acid respiration, 
both state 3 and state 4 respiration rates in the diabetic SSM were significantly decreased ( $P \leq 0.05$; Figure 3.2C), with no change for either state in the IFM (Figure $3.2 \mathrm{D})$.

To determine whether the degree of hyperglycemia and HbA1c level was predictive of the mitochondrial dysfunction we observed in diabetic patients, we generated spline models to determine correlative risk. In Figures 3.3A and 3.3B, state 3 respiration rates were plotted against $\mathrm{HbA1c}$ levels, whereas in Figures $3.3 \mathrm{C}$ and 3.3D, state 3 respiration rates were plotted against blood glucose levels. In each model, we selected a break-point (dashed line) that represented the value at which a diagnosis of diabetes mellitus is performed in a clinical setting. For $\mathrm{HbA1c}$ a value of $6.5 \%$ was selected while for blood glucose, a value of $200 \mathrm{mg} / \mathrm{dL}$ was selected. Both of these values are used to make a diabetic diagnosis $(1,32)$. In Figures $3.3 \mathrm{~A}$ and $3.3 \mathrm{C}$, nondiabetic patients (left of the dashed line) displayed linear correlations suggestive of decreased state 3 respiration rates with increasing HbA1c or blood glucose level. In contrast, diabetic patients (right of the dashed line), displayed linear correlations indicating lower state 3 respiration rates which were similar regardless of increasing HbA1c or blood glucose level (Figures 3.3A and 3.3C). Contrary to SSM, The similar analyses in IFM indicated linear slopes that were more similar between diabetic and non-diabetic patients (Figures 3.3B and 3.3D). 


\section{Electron Transport Chain Complex Activities}

Mitochondrial function was further examined by measuring ETC complex activities. As seen in Figure 3.4, ETC complexes I and IV activities were significantly decreased in the SSM of type 2 diabetic patients relative to non-diabetics $(P \leq 0.05$, Figure 3.4A). ETC complex III did not differ in the SSM of non-diabetic and diabetic patients. ETC complexes I, III and IV activities were not altered in diabetic IFM as compared to non-diabetic IFM (Figure 3.4B).

As above, we determined whether the degree of hyperglycemia and HbA1c level was predictive of the ETC complex I functional decrement observed in diabetic patients, using a spline model. In Figures 3.5A and 3.5B, ETC complex I activities were plotted against HbA1c levels, whereas in Figures 3.5C and 3.5D, ETC complex I activities were plotted against blood glucose levels. In Figures 3.5A and 3.5C, non-diabetic patients (left of the dashed line) displayed linear correlations suggestive of decreased ETC complex I activity with increasing HbA1c or blood glucose level. In contrast, diabetic patients (right of the dashed line), displayed linear correlations indicating lower ETC complex I activity which was similar regardless of increasing HbA1c or blood glucose level (Figures 3.5A and 3.5C). As above, similar analyses in IFM indicated linear slopes that were more similar between diabetic and non-diabetic patients (Figures 3.5B and 3.5D). 


\section{Examination of Electron Transport Chain Complex Protein Expression}

To determine whether loss of ETC complexes was associated with decreased enzymatic activity, we assessed the protein content of ETC complexes by BN-PAGE. Both ETC complex I and complex IV were significantly decreased in the diabetic SSM ( $P \leq 0.05$, Figures 3.6A and 3.7A, respectively). In contrast, no differences in protein content for ETC complexes I or complex IV in the diabetic IFM compared to nondiabetic IFM (Figures 3.6B and 3.7B, respectively). These results suggest a possible mechanism underlying the observed decrease in mitochondrial function of the diabetic SSM compared to the non-diabetic SSM.

\section{Presence of Type 2 Diabetes Mellitus, Coronary Artery Disease and Hypertension}

Because many of the patients studied possessed other comorbidities, we were not able to separate the diabetic patients from the non-diabetic patients without including those with both CAD and hypertension. Nevertheless, we attempted to determine whether the presence of a specific comorbidity was responsible for the observed effects on mitochondrial function. Patients were grouped into three categories: (1) non-diabetic and diabetic patients with both CAD and hypertension; (2) non-CAD and CAD patients without diabetes mellitus; and (3) non-hypertensive and hypertensive patients without diabetes mellitus. These groups were chosen because they enabled us to isolate out the individual comorbidities without the presence of diabetes mellitus (groups 2 and 3) and enable comparison with diabetic and non-diabetic patients who possessed both comorbidities (group 1). Table 3.2 displays the mitochondrial functional analyses of non-diabetic and diabetic patients who all have CAD and hypertension and 
the data shows a decrease in both mitochondrial respiration and ETC complex I and IV activity of diabetic patients who have CAD and hypertension. Table 3.3 represents mitochondrial functional analyses of patients who had or did not have CAD, of which none of the patients were hypertensive or diabetic and results indicated no observed mitochondrial dysfunction in the patients who possessed CAD. Table 3.4 illustrates the mitochondrial functional analyses of patients who were or were not hypertensive, of which none had CAD or were diabetic and similarly, data demonstrated that the mitochondrial function of these hypertensive patients. These results suggest that the mitochondrial dysfunction observed in type 2 diabetic patients who have both CAD and hypertension is not due to either CAD or hypertension independently.

\section{The Influence of Body Mass Index (BMI) on Type 2 Diabetes Mellitus}

We sought to determine the influence of BMI on SSM mitochondrial function during type 2 diabetes mellitus using a similar spline approach. The linear spline models in Figures 3.8A and 3.8B include a break-point at an $\mathrm{HbA1c}$ level equal to 6.5\% (dashed line). Based on the World Health Organization, patients whose BMI is less than 30 $\mathrm{kg} / \mathrm{m}^{2}$ are considered non-obese, whereas patients whose BMI is greater than $30 \mathrm{~kg} / \mathrm{m}^{2}$ are considered obese (31). Patients with a $\mathrm{BMI}<30 \mathrm{~kg} / \mathrm{m}^{2}$ are indicated in white while those patients with a BMI $>30 \mathrm{~kg} / \mathrm{m}^{2}$ are indicated in black (Figures $3.8 \mathrm{~A}$ and $3.8 \mathrm{~B}$ ). After categorizing the patients as non-obese or obese, we were able to visualize the impact of obesity on these two measurements of mitochondrial function (Figure 3.8). If obesity were impacting mitochondrial function to a great extent, we would expect to see the obese patients clustered at the bottom of the spline model, indicating a lower 
mitochondrial function compared to the non-obese patients. Interestingly, we observed an even displacement of the obese patients throughout the patients' data, indicating that obesity does not independently impact mitochondrial function, specifically SSM state 3 respiration and SSM ETC complex I activity as measured by HbA1c levels. Our results indicated that SSM state 3 respiration (Figure 3.8A) and SSM ETC complex I activity (Figure 3.8B) are not correlated with a patient's BMI, regardless of diabetic status. These findings suggest that the BMI level of an individual is not an effective predictor of type 2 diabetic status nor mitochondrial dysfunction. 


\section{DISCUSSION}

Mitochondrial dysfunction contributes to cardiovascular complications that are associated with type 2 diabetes mellitus $(13,19,24,25)$. The existence of two mitochondrial subpopulations present in the cardiomyocyte further complicates mitochondrial functional analyses. Our laboratory and others have reported that these two separate pools of mitochondria are affected differently by pathologies, such as diabetes mellitus in rodent models $(13,14,21,22)$. Limited research has used a human heart model to examine mitochondrial function (30) and the effects of diabetes mellitus $(3,4)$. The goal of this translational study was to determine how type 2 diabetes mellitus affects individual mitochondrial subpopulations in human patients. Our results suggest that mitochondrial dysfunction is present in the heart of human type 2 diabetic patients, with the SSM being affected to a greater extent than the IFM, as demonstrated by decreased glucose- and fatty acid-mediated respiration, ETC complex I and IV activities and complex expression.

With our large data set, we were able to analyze relationships between nondiabetics and diabetics by examining state 3 mitochondrial respiration and ETC complex I activities. By utilizing linear regression spline models for state 3 mitochondrial respiration as well as ETC complex I activity and separating non-diabetic and diabetic patients by HbA1c levels, a decreasing slope for non-diabetic patients suggested that as HbA1c level increases, SSM state 3 respiration and ETC complex I activity decreases. Once an $\mathrm{HbA} 1 \mathrm{c}$ level of $6.5 \%$ is reached, the slope of the linear regression 
remains somewhat flat despite further increases in $\mathrm{HbA1c}$ levels. These findings indicate that mitochondrial dysfunction is already present in diabetics with $\mathrm{HbA} 1 \mathrm{c}$ levels in the modestly elevated range and that as HbA1c levels increase the extent of mitochondrial dysfunction remains the same. A similar phenomenon is apparent when analyzing SSM state 3 respiration and SSM ETC complex I activity and applying a spline model which utilizes a blood glucose level of $200 \mathrm{mg} / \mathrm{dL}$. Taken together these findings indicate that mitochondrial dysfunction in the SSM of type 2 diabetic patients is present despite the degree of control over key indices that are predictive of patient morbidity, such as HbA1c and blood glucose levels. Further, these findings may suggest that the presence of the diabetic phenotype independent of the stage or timing of the disease is associated with dysfunctional profiles to cardiac mitochondria.

When analyzing the spline models of state 3 respiration and ETC complex activity I measured by blood glucose levels, similar relationships are observed as compared to examining these mitochondrial functional parameters measured by $\mathrm{HbA1c}$ levels. In regards to SSM state 3 respiration, the linear regression slope before a blood glucose level of $200 \mathrm{mg} / \mathrm{dL}$ is noticeably decreasing, with no visible slope for the diabetic patients whose blood glucose levels are higher than $200 \mathrm{mg} / \mathrm{dL}$. By comparing the two relationships of SSM state 3 mitochondrial respiration between non-diabetic and diabetic patients, we were able to determine that SSM state 3 respiration decreased with increasing blood glucose levels until a blood glucose level of $200 \mathrm{mg} / \mathrm{dL}$ is reached, from which no correlation between mitochondrial dysfunction and increasing blood glucose levels existed. The spline model of SSM ETC complex activity I measured by 
blood glucose levels also revealed a similar phenomenon; however, the linear regression slope was not as dramatic as with SSM state 3 respiration. With that being said, SSM ETC complex I activity decreased with increasing blood glucose levels until a blood glucose level of $200 \mathrm{mg} / \mathrm{dL}$ was reached. Past this threshold, SSM ETC complex I activity dysfunction remained constant, regardless of increasing blood glucose levels.

In contrast to our current study, Rosca et al. reported increased ETC complex I and complex III expression levels in the SSM, with no change in ETC complex IV in a canine coronary heart failure model as measured by BN-PAGE (27). It is unclear why the results from our study differed by may be due in part to differences in the pathologies, heart failure vs. diabetes mellitus, as well as the examination of canine and human models. Anderson et al (4) reported no change in ETC complexes I-V expression in diabetic patients when compared to non-diabetics. This difference may be due to the experimental procedures employed. Anderson et al. examined polypeptides from denatured proteins as opposed to the protein expression of the individual complex as a whole unit, which was the case in our study. This difference could also be explained by the examination of homogenate versus mitochondrial subpopulations used in the current study.

Examining mitochondrial function in the human heart does not come without critical limitations. First, the biopsies are collecting come from individuals undergoing cardiac procedures, which may impart negative effects on the heart. Despite this complication, we feel that the non-diabetic group of patients is the appropriate group to 
compare with the diabetic patients because the clinical variables and medications that may influence the results are no longer taken into consideration due to the fact that the both groups have similar clinical variables, such as CAD, hypertension, and medications (Table 3.1).

The presence of other clinical co-morbidities has been a factor in the examination of the human heart because most of the patients who are undergoing cardiac procedures are diagnosed with a multitude of compounding diseases, such as CAD, type 2 diabetes mellitus and hypertension. Although the patients that were diagnosed with these illnesses were similarly distributed in both groups (Table 3.1), it is unclear as to which disease was affecting the mitochondrial functional outcomes. In this study, we were able to determine that $\mathrm{CAD}$, hypertension nor $\mathrm{BMI}$ alone were the prominent contributing factor to the observed mitochondrial dysfunction (Tables 3.3 and 3.4, and Figure 3.8, respectively) in patients with type 2 diabetes mellitus. Our data is in agreement with Anderson et al. (4) and indicates that type 2 diabetes mellitus, in the presence of $C A D$ and hypertension, is part of a multifaceted interaction that all together contributes to mitochondrial dysfunction (Table 3.2). Although we had a large patient number, we were unable to separate the effects of type 2 diabetes mellitus alone on mitochondrial functionality.

In conclusion, we are the first to demonstrate mitochondrial subpopulationspecific dysfunction in the human heart of type 2 diabetic patients, with the SSM being impacted to a greater extent than the IFM. With the use of linear spline models, we were 
able to illustrate that once a threshold is reached, either with $\mathrm{HbA1c}$ or blood glucose levels, mitochondrial dysfunction is no longer correlated with decreasing levels, highlighting the importance of preserving mitochondrial function regardless of the stage of type 2 diabetic onset. 


\section{ACKNOWLEDGEMENTS}

I would like to acknowledge Ruby Memorial Hospital and West Virginia University School of Medicine, Department of Surgery and the West Virginia Clinical and Translational Science Institute, Department of Biostatistics School of Public Health for their collaboration and contribution to this study.

This work was supported by the National Institutes of Health from the National Institutes of Diabetes and Digestive and Kidney Diseases [DP2DK083095]. This work was also supported by a Grant-In-Aid from the American Heart Association [0855484D]. Tara Croston is a recipient of an NIH Predoctoral Fellowship [T32HL090610]. Danielle Shepherd is a recipient of an NIH Predoctoral Fellowship [T32HL090610]. Cody Nichols is a recipient of an Integrative Graduate Education and Research Traineeship Program [DGE-1144676]. Dustin Long is supported by the National Institute of General Medical Sciences of the National Institutes of Health under Award Number U54GM104942. Flow cytometry experiments were performed in the West Virginia University Flow Cytometry Core Facility, which is supported in part the Institutional Development Award (IDeA)

from the National Institute of General Medical Sciences of the National Institutes of Health under grant numbers [P30GM103488] (CoBRE) and [RCP11011809] (INBRE). 


\section{REFERENCES}

1. ADA. Diabetes Basics, 2013.

2. An D and Rodrigues B. Role of changes in cardiac metabolism in development of diabetic cardiomyopathy. American Journal of Physiology - Heart and Circulatory Physiology 291: H1489-H1506, 2006.

3. Anderson EJ, Kypson AP, Rodriguez E, Anderson CA, Lehr EJ, and Neufer PD. Substrate-Specific Derangements in Mitochondrial Metabolism and Redox Balance in the Atrium of the Type 2 Diabetic Human Heart. Journal of the American College of Cardiology 54: 1891-1898, 2009.

4. Anderson EJ, Rodriguez E, Anderson CA, Thayne K, Chitwood WR, and Kypson AP. Increased propensity for cell death in diabetic human heart is mediated by mitochondrial-dependent pathways. American Journal of Physiology - Heart and Circulatory Physiology 300: H118-H124, 2011.

\section{Baseler WA, Dabkowski ER, Williamson CL, Croston TL, Thapa D, Powell}

MJ, Razunguzwa TT, and Hollander JM. Proteomic alterations of distinct mitochondrial subpopulations in the type 1 diabetic heart: contribution of protein import dysfunction. Am J Physiol Regul Integr Comp Physiol 300: R186-200, 2011.

6. Belke DD, Larsen TS, Gibbs EM, and Severson DL. Altered metabolism causes cardiac dysfunction in perfused hearts from diabetic (db/db) mice. Am J Physiol Endocrinol Metab 279: E1104-1113, 2000.

7. Boudina S and Abel ED. Mitochondrial uncoupling: a key contributor to reduced cardiac efficiency in diabetes. Physiology (Bethesda) 21: 250-258, 2006. 
8. Boudina S, Sena S, Theobald H, Sheng X, Wright JJ, Hu XX, Aziz S, Johnson Jl, Bugger H, Zaha VG, and Abel ED. Mitochondrial energetics in the heart in obesity-related diabetes: direct evidence for increased uncoupled respiration and activation of uncoupling proteins. Diabetes 56: 2457-2466, 2007.

9. Bradford MM. A rapid and sensitive method for the quantitation of microgram quantities of protein utilizing the principle of protein-dye binding. Anal Biochem 72: 248254, 1976.

10. CDC. National diabetes fact sheet: national estimates and general information on diabetes and prediabetes in the United States, 2011. : Atlanta, GA: U.S. Department of Health and Human Services, Centers for Disease Control and Prevention, 2011.

11. Chance B and Williams GR. Respiratory enzymes in oxidative phosphorylation. I. Kinetics of oxygen utilization. J Biol Chem 217: 383-393, 1955.

12. Chance B and Williams GR. Respiratory enzymes in oxidative phosphorylation. VI. The effects of adenosine diphosphate on azide-treated mitochondria. J Biol Chem 221: 477-489, 1956.

13. Dabkowski ER, Baseler WA, Williamson CL, Powell M, Razunguzwa TT, Frisbee JC, and Hollander JM. Mitochondrial dysfunction in the type 2 diabetic heart is associated with alterations in spatially distinct mitochondrial proteomes. Am J Physiol Heart Circ Physiol 299: H529-540, 2010.

14. Dabkowski ER, Baseler WA, Williamson CL, Powell M, Razunguzwa TT, Frisbee JC, and Hollander JM. Mitochondrial dysfunction in the type 2 diabetic heart is associated with alterations in spatially distinct mitochondrial proteomes. Am J Physiol Heart Circ Physiol 299: H529-540. 
15. Dabkowski ER, Williamson CL, Bukowski VC, Chapman RS, Leonard SS, Peer CJ, Callery PS, and Hollander JM. Diabetic cardiomyopathy-associated dysfunction in spatially distinct mitochondrial subpopulations. Am J Physiol Heart Circ Physiol 296: H359-369, 2009.

16. Dabkowski ER, Williamson CL, and Hollander JM. Mitochondria-specific transgenic overexpression of phospholipid hydroperoxide glutathione peroxidase (GPx4) attenuates ischemia/reperfusion-associated cardiac dysfunction. Free Radic Biol Med 45: 855-865, 2008.

17. Garcia MJ, McNamara PM, Gordon T, and Kannel WB. Morbidity and mortality in diabetics in the Framingham population. Sixteen year follow-up study. Diabetes 23: 105-111, 1974.

18. Hofhaus G, Shakeley RM, and Attardi G. Use of polarography to detect respiration defects in cell cultures. Methods Enzymol 264: 476-483, 1996.

19. Kelley DE, He J, Menshikova EV, and Ritov VB. Dysfunction of mitochondria in human skeletal muscle in type 2 diabetes. Diabetes 51: 2944-2950, 2002.

20. Leahy JL. Pathogenesis of Type 2 Diabetes Mellitus. Archives of Medical Research 36: 197-209, 2005.

21. Lesnefsky EJ, Slabe TJ, Stoll MS, Minkler PE, and Hoppel CL. Myocardial ischemia selectively depletes cardiolipin in rabbit heart subsarcolemmal mitochondria. Am J Physiol Heart Circ Physiol 280: H2770-2778, 2001.

22. Moghaddas S, Stoll MS, Minkler PE, Salomon RG, Hoppel CL, and Lesnefsky EJ. Preservation of cardiolipin content during aging in rat heart interfibrillar mitochondria. J Gerontol A Biol Sci Med Sci 57: B22-28, 2002. 
23. Palmer JW, Tandler B, and Hoppel CL. Biochemical properties of subsarcolemmal and interfibrillar mitochondria isolated from rat cardiac muscle. Journal of Biological Chemistry 252: 8731-8739, 1977.

24. Rabol R, Boushel R, and Dela F. Mitochondrial oxidative function and type 2 diabetes. Appl Physiol Nutr Metab 31: 675-683, 2006.

25. Ritov VB, Menshikova EV, He J, Ferrell RE, Goodpaster BH, and Kelley DE. Deficiency of subsarcolemmal mitochondria in obesity and type 2 diabetes. Diabetes 54 : 8-14, 2005.

26. Rolo AP and Palmeira CM. Diabetes and mitochondrial function: role of hyperglycemia and oxidative stress. Toxicol Appl Pharmacol 212: 167-178, 2006.

27. Rosca MG, Vazquez EJ, Kerner J, Parland W, Chandler MP, Stanley W, Sabbah HN, and Hoppel CL. Cardiac mitochondria in heart failure: decrease in respirasomes and oxidative phosphorylation. Cardiovasc Res 80: 30-39, 2008.

28. Semeniuk LM, Kryski AJ, and Severson DL. Echocardiographic assessment of cardiac function in diabetic $\mathrm{db} / \mathrm{db}$ and transgenic $\mathrm{db} / \mathrm{db}$-hGLUT4 mice. Am J Physiol Heart Circ Physiol 283: H976-982, 2002.

29. Trounce IA, Kim YL, Jun AS, and Wallace DC. Assessment of mitochondrial oxidative phosphorylation in patient muscle biopsies, lymphoblasts, and transmitochondrial cell lines. Methods Enzymol 264: 484-509, 1996.

30. Weinstein ES, Benson DW, and Fry DE. Subpopulations of human heart mitochondria. Journal of Surgical Research 40: 495-498, 1986.

31. WHO. BMI classification., 2013. 
32. WHO. Use of Glycated Haemoglobin (HbA1c) in the Diagnosis of Diabetes Mellitus 2011.

33. Williamson CL, Dabkowski ER, Baseler WA, Croston TL, Alway SE, and Hollander JM. Enhanced apoptotic propensity in diabetic cardiac mitochondria: influence of subcellular spatial location. Am J Physiol Heart Circ Physiol 298: H633-642, 2010. 
Figure 3.1

A.

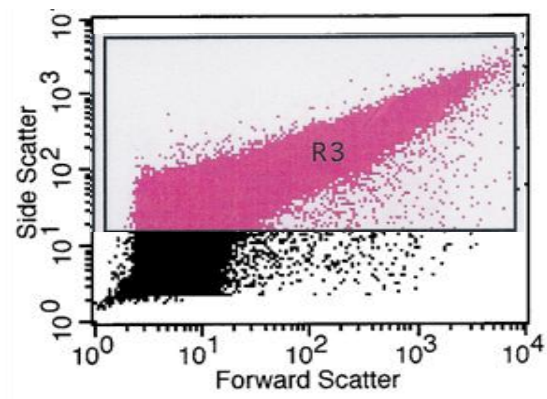

C.

Mitochondrial Size

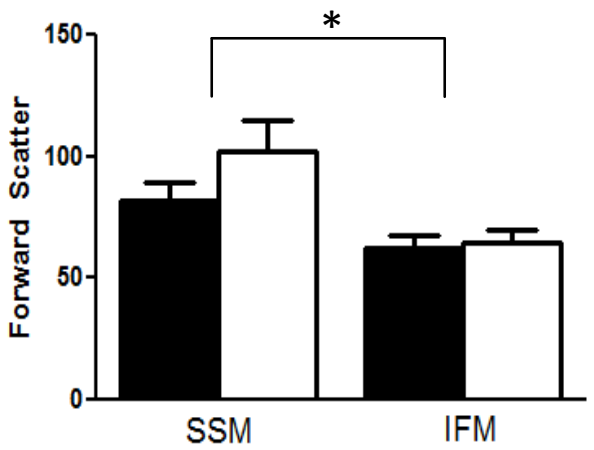

B.

IFM Control

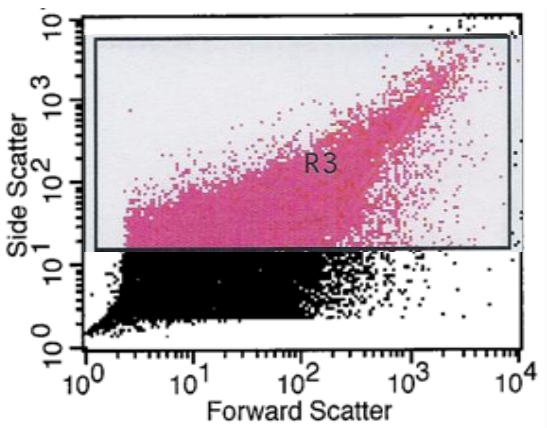

D. Internal Complexity

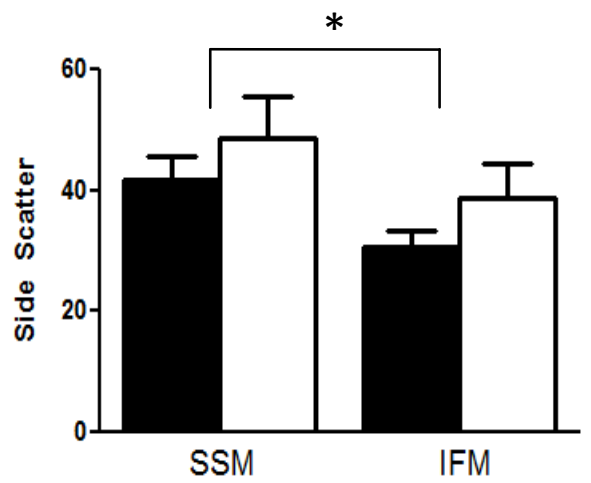

E. Mitochondrial Membrane Potential

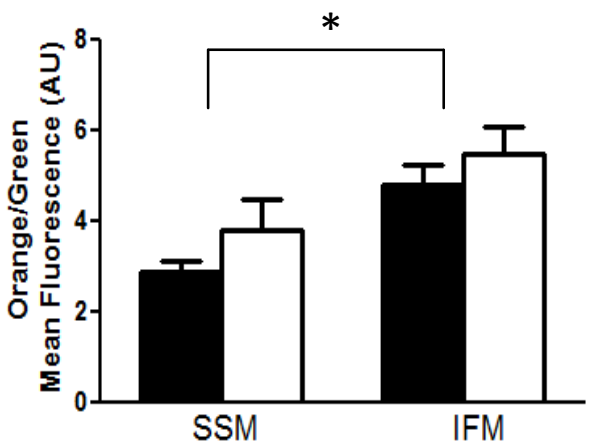


Figure 3.1 Mitochondrial characteristics. Cardiac mitochondrial subpopulations were isolated and incubated with JC-1 dye. Forward scatter and side scatter were used to analyze the isolated mitochondria as seen by a (A) representative histogram of nondiabetic SSM; and a (B) representative histogram of non-diabetic IFM. Mitochondrial size (C) and internal complexity (D) was quantified for each mitochondrial subpopulation. By calculating the ratio of green to orange fluorescence, membrane potential (E) was quantified for non-diabetic and diabetic SSM and IFM. Closed bars represent non-diabetic patients and open bars represent diabetic patients. ${ }^{*} \mathrm{P} \leq 0.05$ SSM non-diabetic vs. IFM non-diabetic; $N=28$ non-diabetics and 23 diabetics. 
Figure 3.2

A.

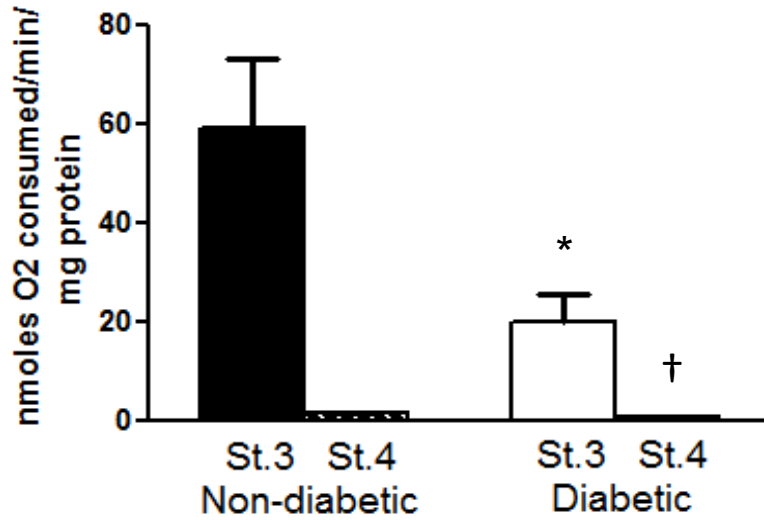

C.

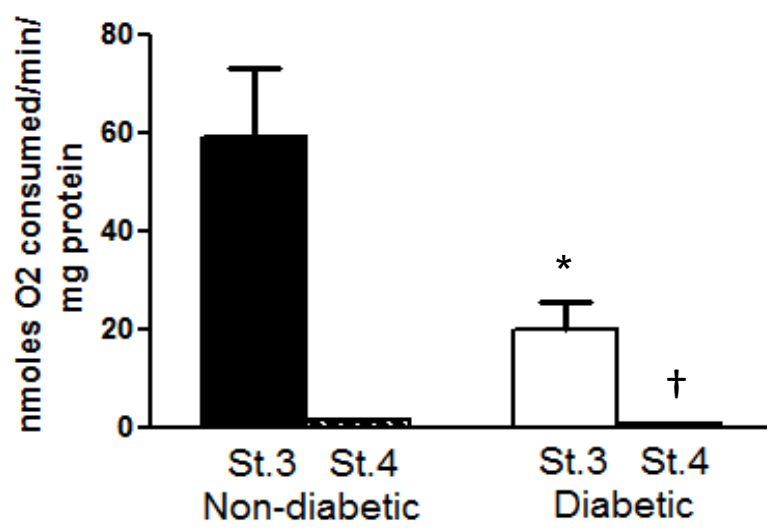

B.

IFM Respiration

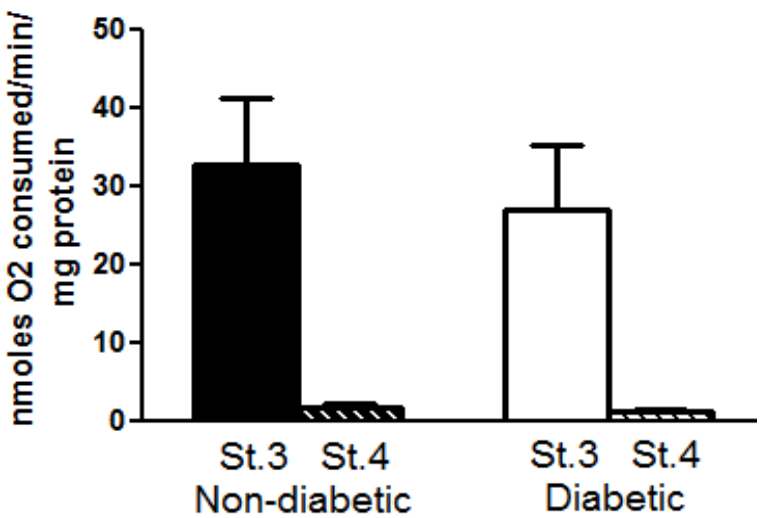

D.

IFM Respiration

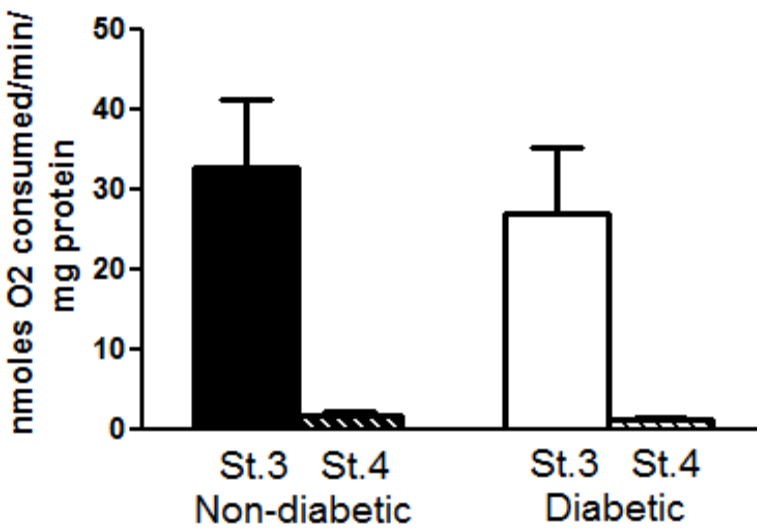


Figure 3.2 Mitochondrial subpopulation respiration rates. Cardiac mitochondrial subpopulations were isolated and respiration rates were measured using different substrates. Glutamate and malate were used as substrates to measure the amount of oxygen consumed (State 3 and State 4) for (A) non-diabetic and diabetic SSM and (B) non-diabetic and diabetic IFM ( $N=28$ non-diabetics and 23 diabetics $)$. Palmitoylcarnitine and malate were used as substrates to measure the amount of oxygen consumed (State 3 and State 4) for (C) non-diabetic and diabetic SSM and (D) non-diabetic and diabetic IFM ( $N=17$ non-diabetics and 10 diabetics). Values are mean \pm SEM. Units are nmoles $\mathrm{O}_{2}$ consumed $/ \mathrm{min} / \mathrm{mg}$ of protein. Closed bars represent nondiabetic patients and open bars represent diabetic patients. ${ }^{*} \mathrm{P} \leq 0.05$ State 3 SSM nondiabetic vs. State 3 SSM diabetic. $† \mathrm{P} \leq 0.05$ State 4 SSM non-diabetic vs. State 4 SSM diabetic. 
Figure 3.3

A.

SSM State 3 Respiration measured by HbA1c levels

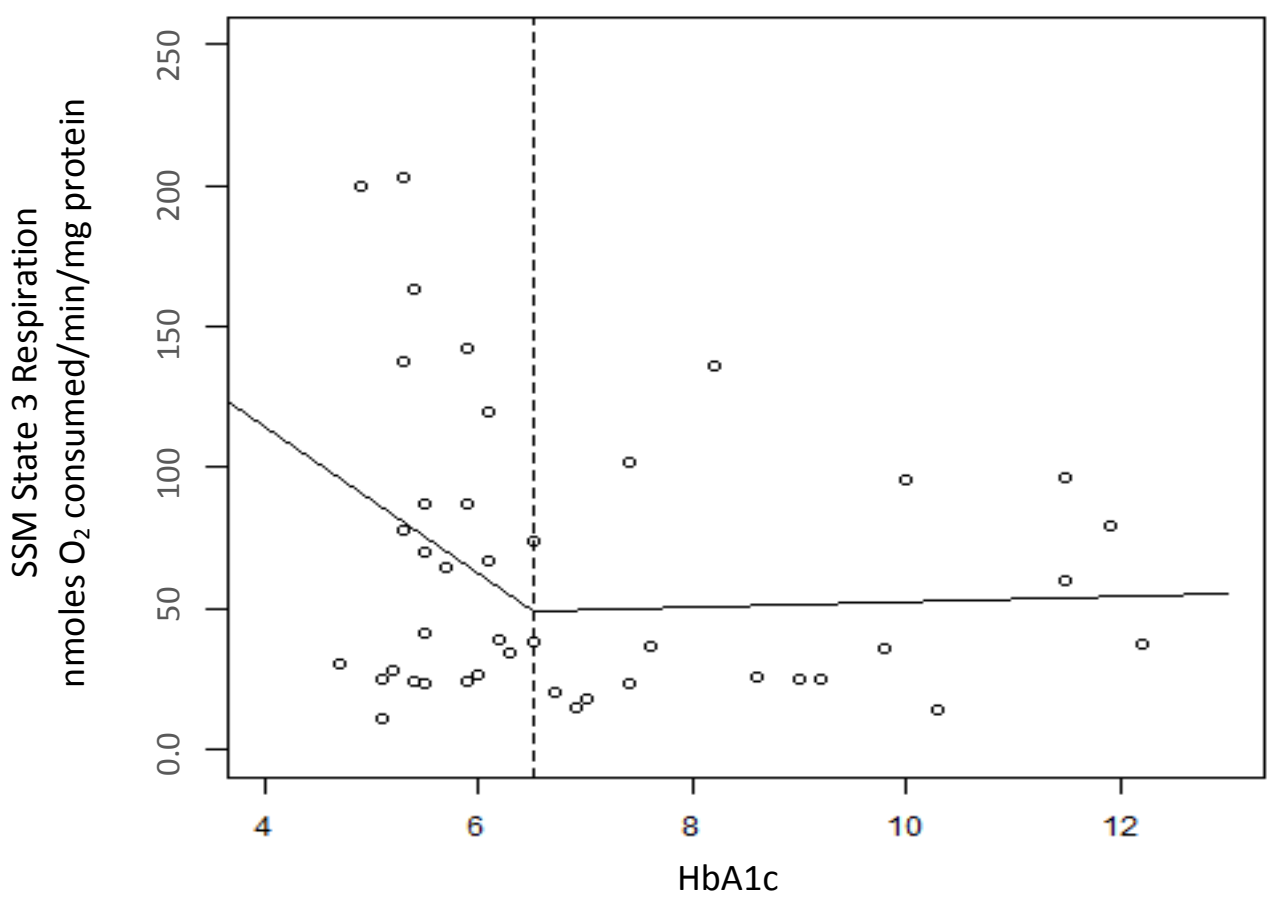

B.

IFM State 3 Respiration measured by HbA1c levels

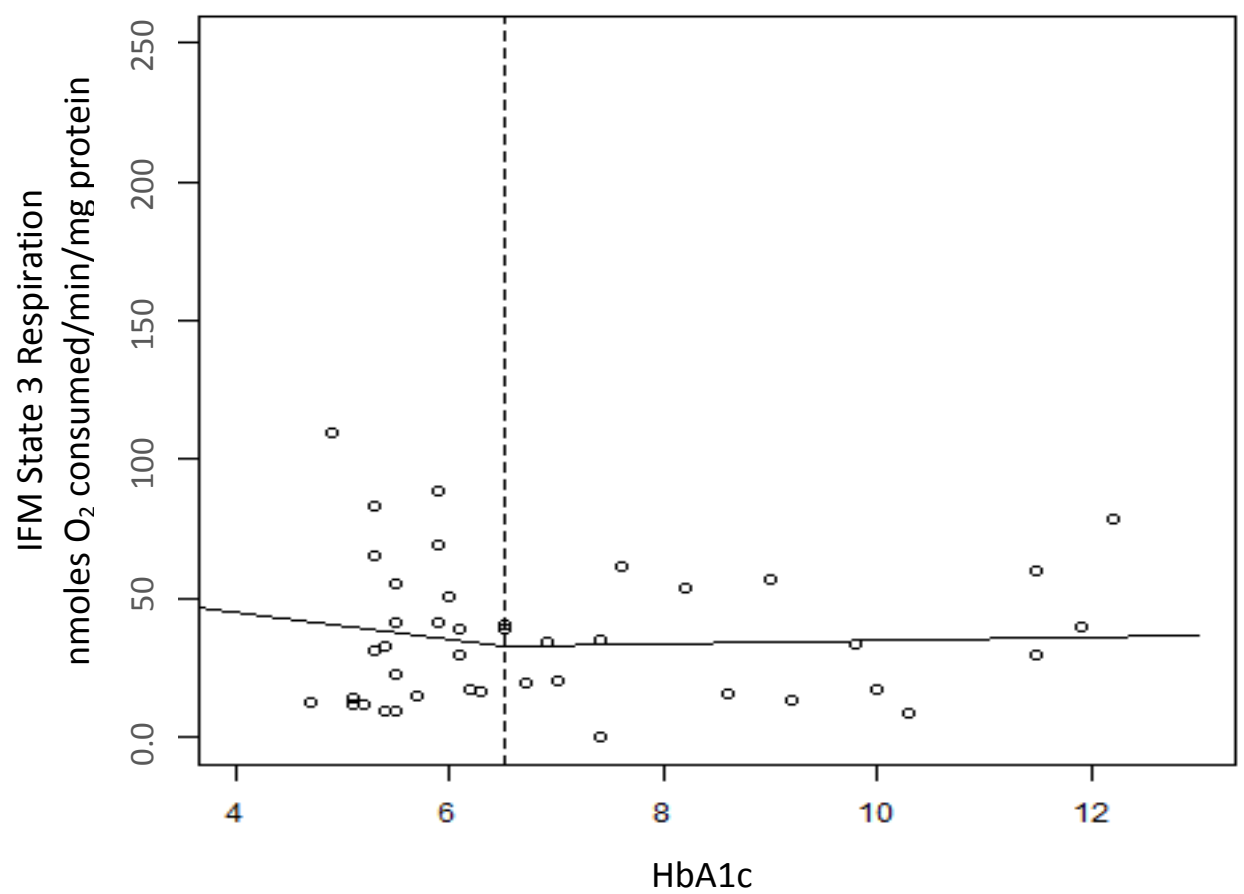


C.

SSM State 3 Respiration measured by blood glucose levels

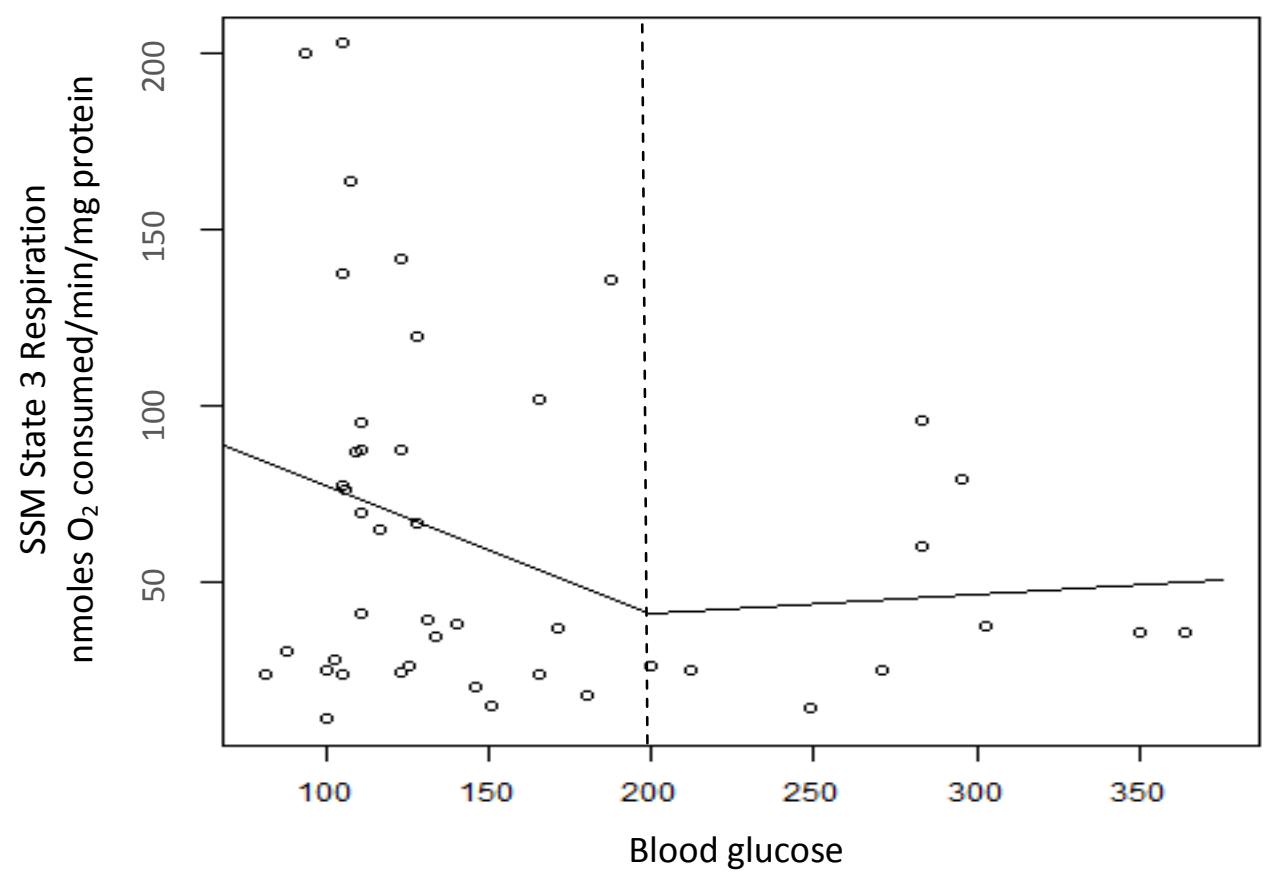

D.

IFM State 3 Respiration measured by blood glucose levels

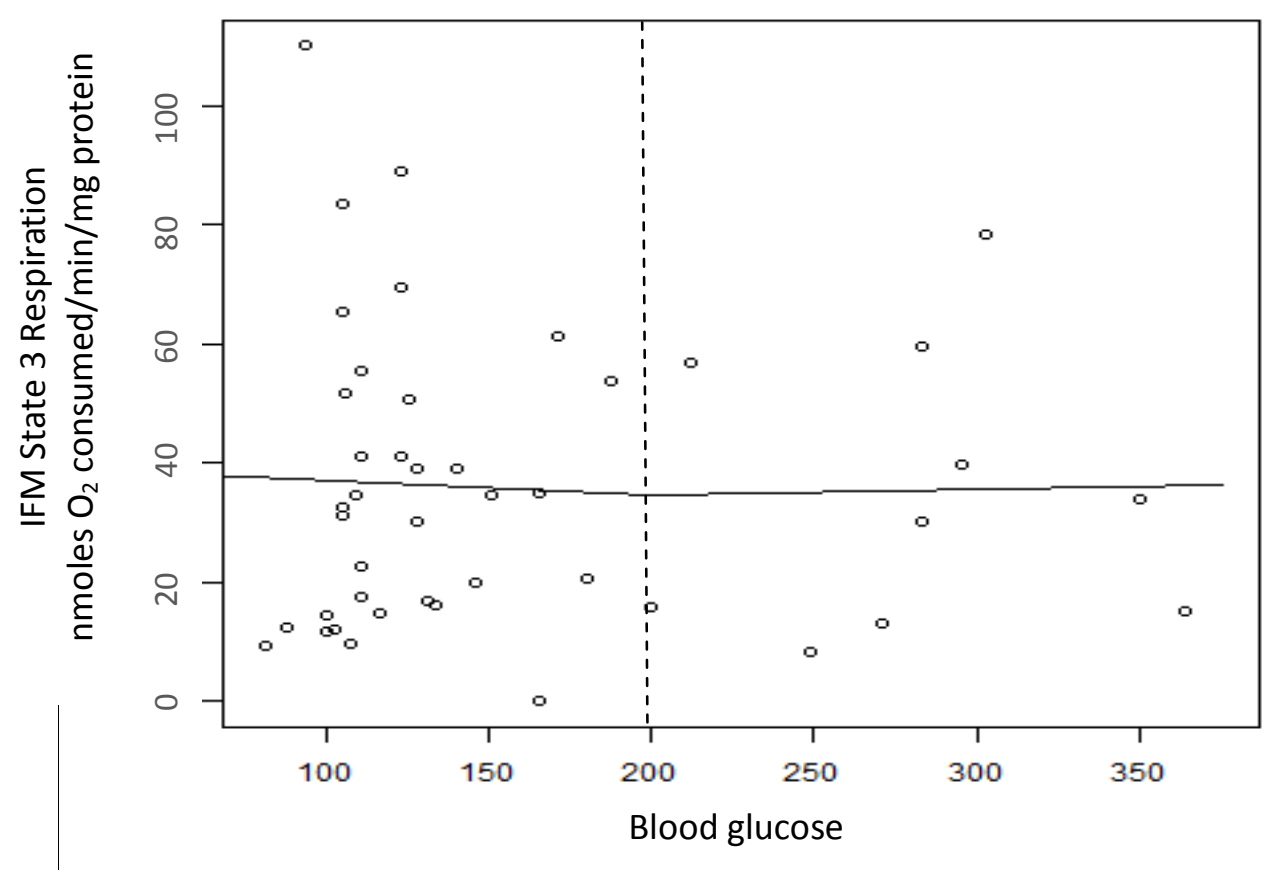


Figure 3.3 Linear spline illustrations for mitochondrial respiration. Linear spline models for $\mathrm{HbA} 1 \mathrm{c}$ and mitochondrial respiration, specifically state 3 , were performed with a break-point at an $\mathrm{HbA} 1 \mathrm{c}$ level equal to $6.5 \%$ (dashed line). The linear relationship before and after the break-point are plotted for SSM (A) and IFM (B) with dots representing each patient with an observed HbA1c level. A linear spline model for blood glucose and state 3 mitochondrial respiration was performed with a break-point at a blood glucose level equal to $200 \mathrm{mg} / \mathrm{dL}$ (dashed line). The linear relationship before and after the break-point are plotted for SSM (C) and IFM (D) with dots representing each patient with an observed blood glucose level. Units are nmoles $\mathrm{O}_{2}$ consumed/min/mg of protein. 
Figure 3.4

A.

SSM

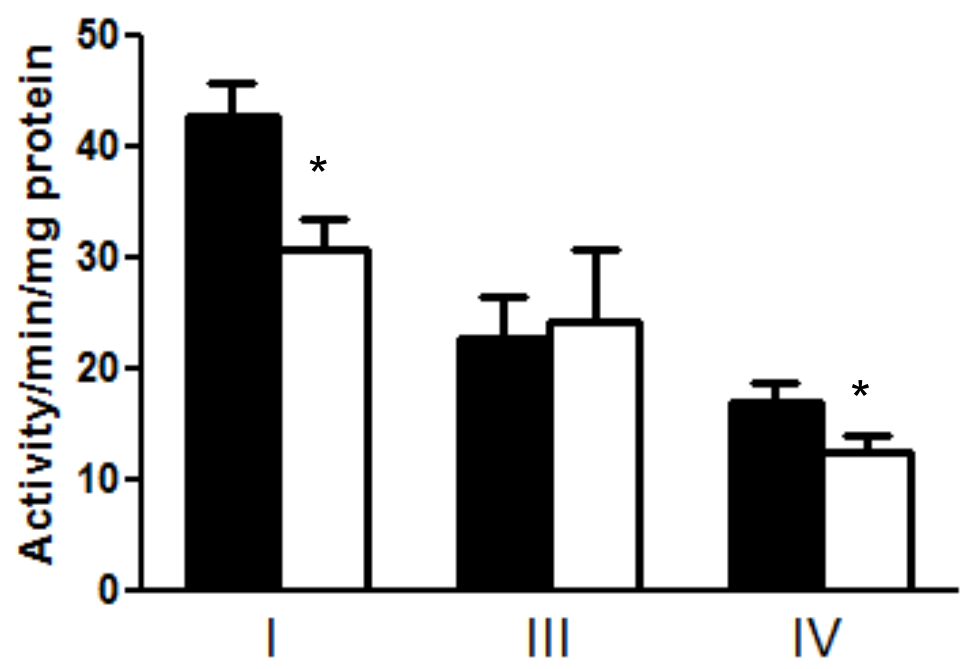

B.

IFM

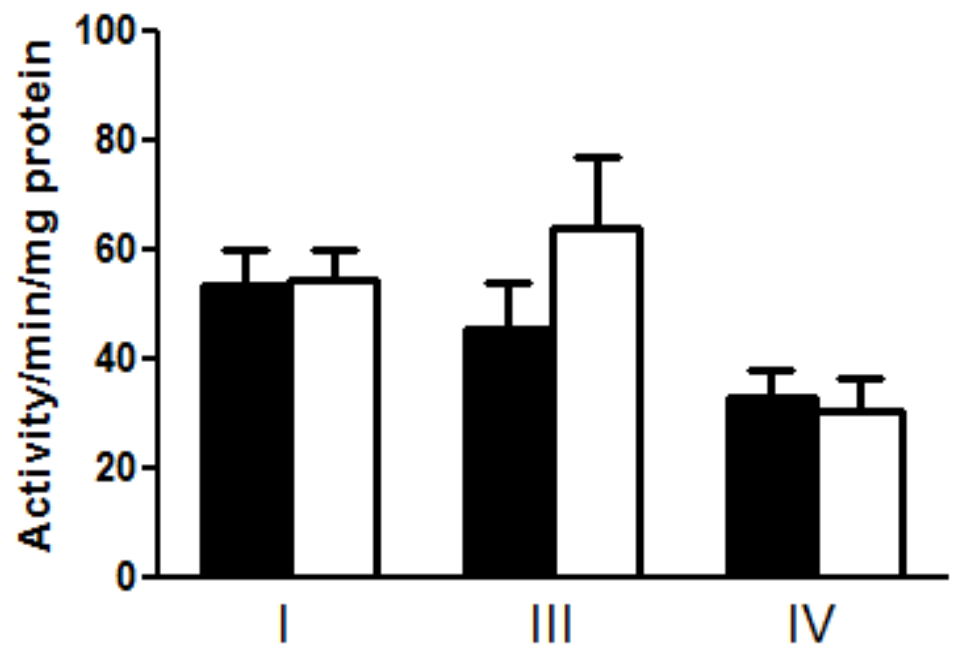


Figure 3.4 Electron transport chain activities in mitochondrial subpopulations. Cardiac mitochondrial subpopulations were isolated and ETC complex I, III, and IV activities were measured. ETC complexes I, III, and IV in the non-diabetic and diabetic SSM (A) and IFM (B). Values are mean \pm SEM. Units are activity $/ \mathrm{min} / \mathrm{mg}$ of protein. Closed bars represent non-diabetic patients and open bars represent diabetic patients. ${ }^{*} \mathrm{P} \leq 0.05$ SSM non-diabetic vs. SSM diabetic; $N=47$ non-diabetics and 34 diabetics. 
Figure 3.5

A.

SSM ETC Complex I Activity measured by HbA1c levels

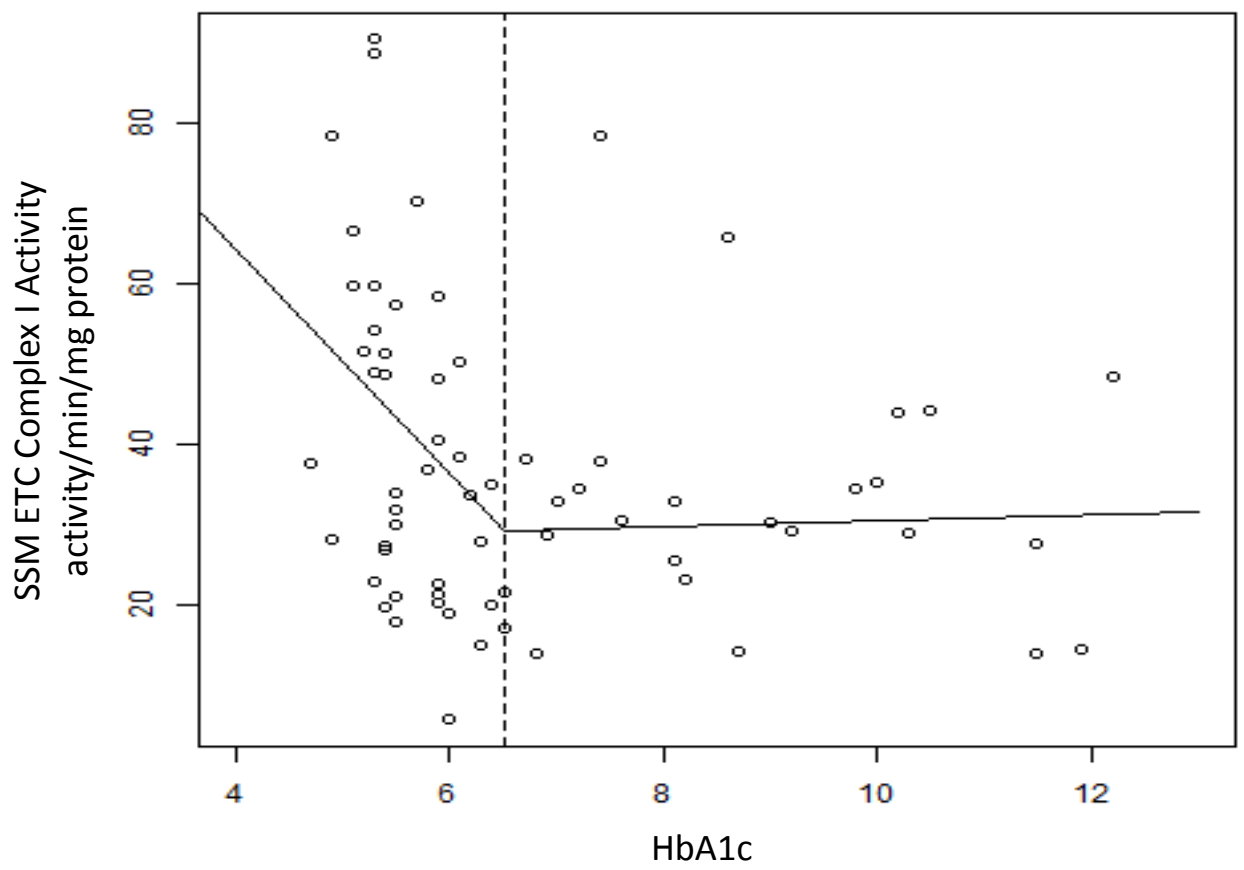

B.

IFM ETC Complex I Activity measured by HbA1c levels

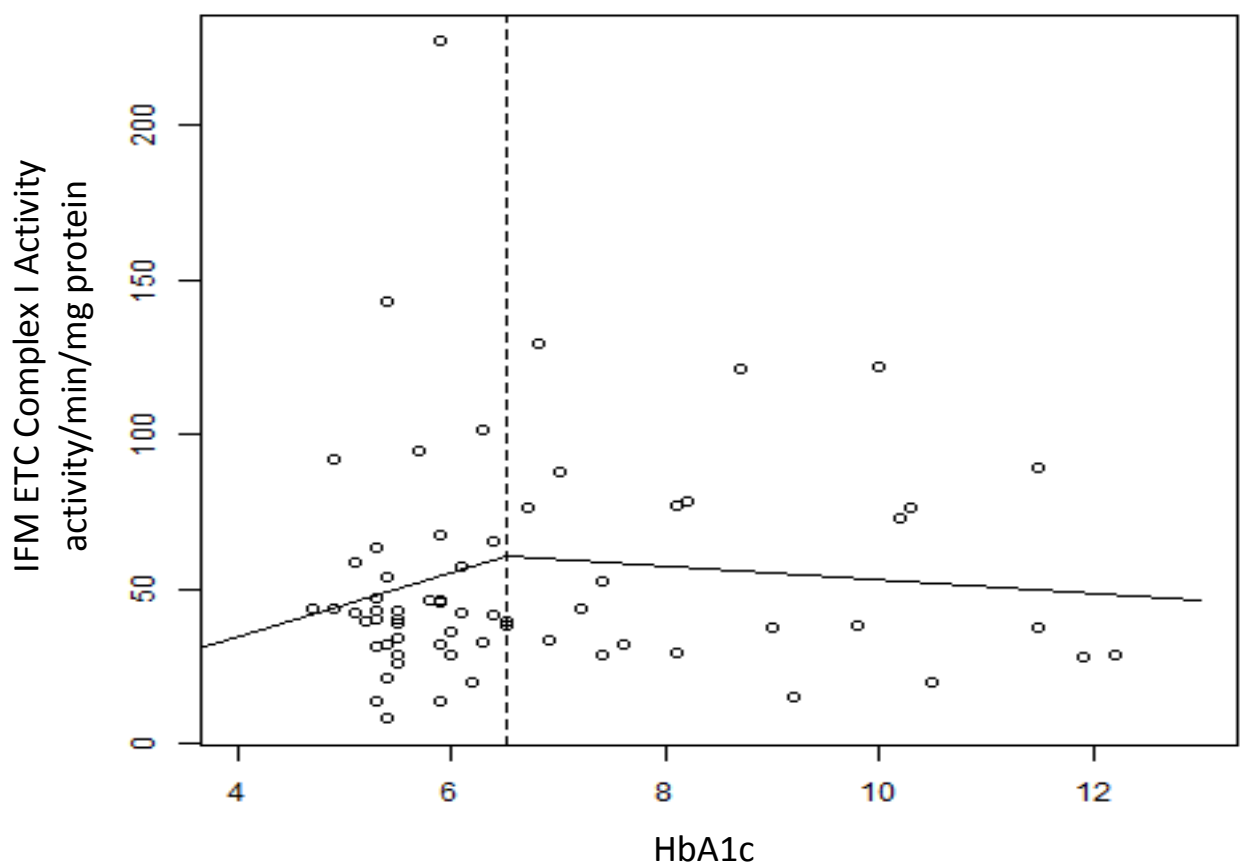


C.

SSM ETC Complex I Activity measured by blood glucose levels

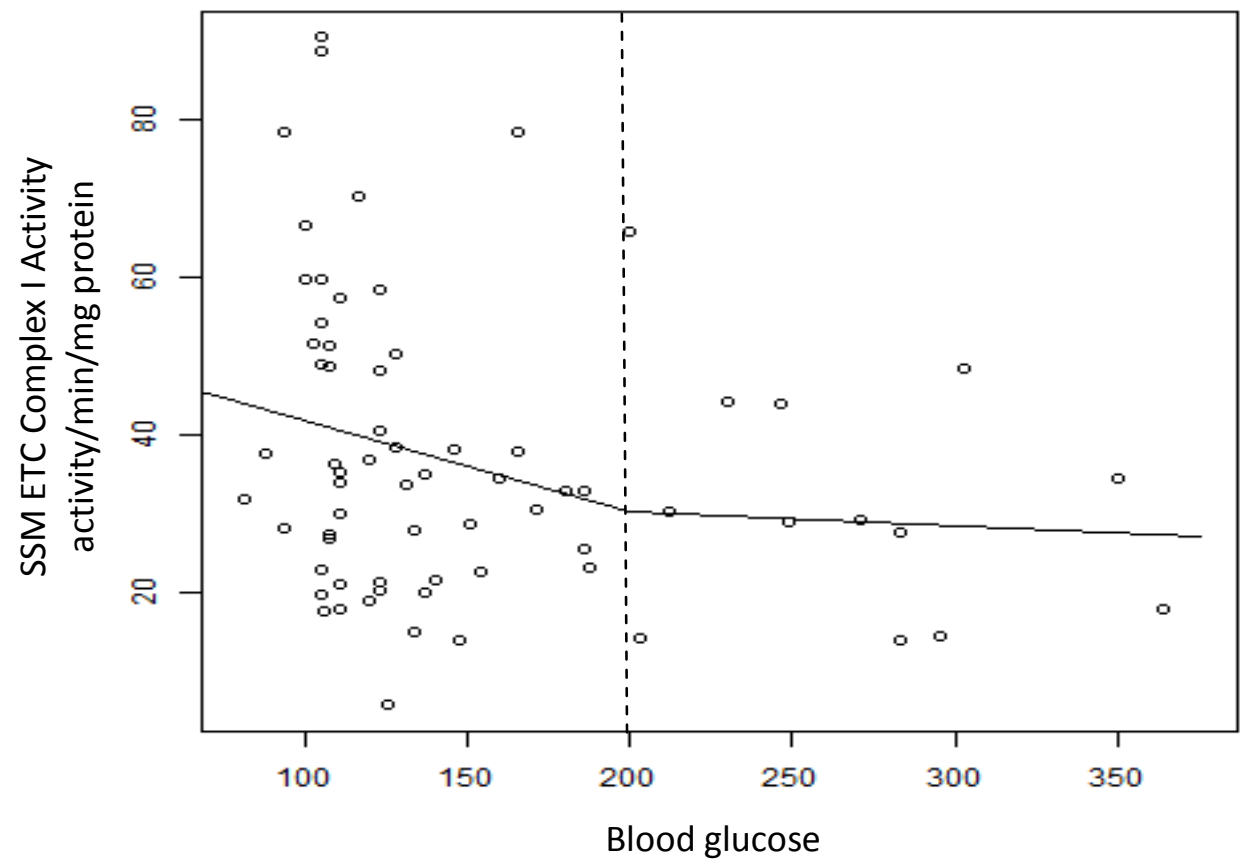

D.

IFM ETC Complex I Activity measured by blood glucose levels

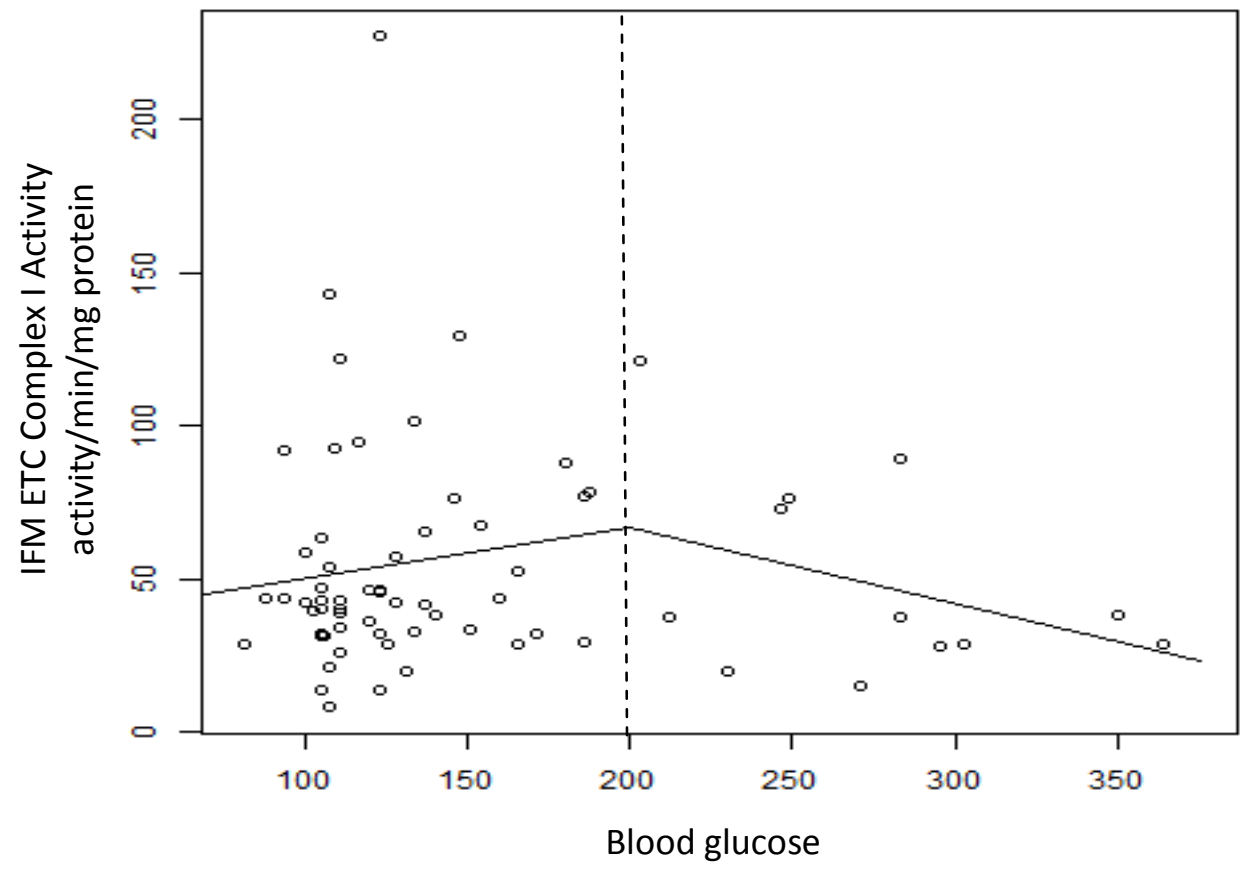


Figure 3.5 Linear spline illustrations for electron transport chain complex I activity. Linear spline models for HbA1c and ETC complex I activity, were performed with a break-point at an $\mathrm{HbA} 1 \mathrm{c}$ level equal to $6.5 \%$ (dashed line). The linear relationship before and after the break-point are plotted for SSM (A) and IFM (B) with dots representing each patient with an observed HbA1c level. A linear spline model for blood glucose and ETC complex I activity was performed with a break-point at blood glucose level equal to $200 \mathrm{mg} / \mathrm{dL}$ (dashed line). The linear relationship before and after the break-point are plotted for SSM (C) IFM (D) with dots representing each patient with an observed blood glucose level. Units are activity/min/mg of protein. 
Figure 3.6

A. SSM Complex I Blue Native

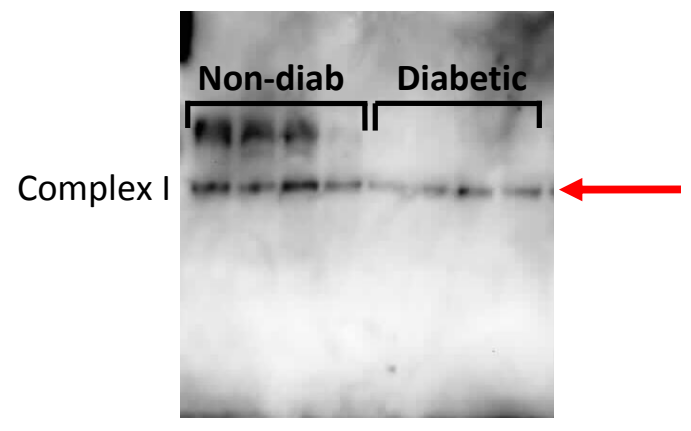

C. IFM Complex I Blue Native

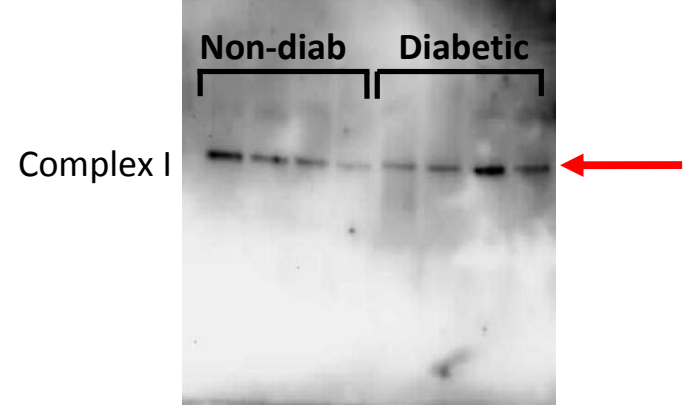

B. SSM: Complex I

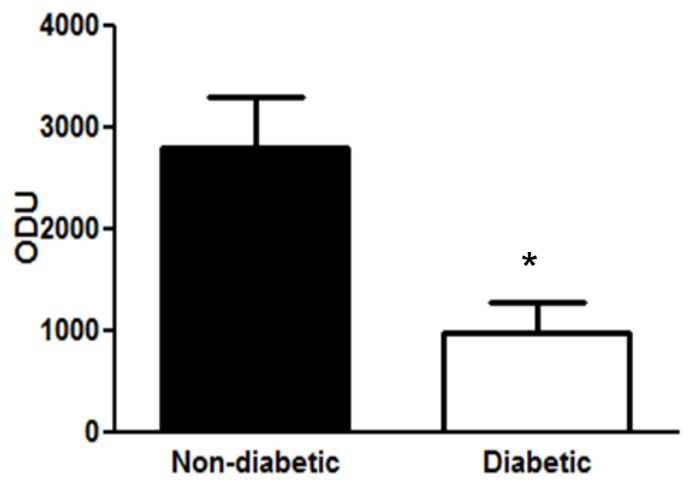

D. IFM: Complex I

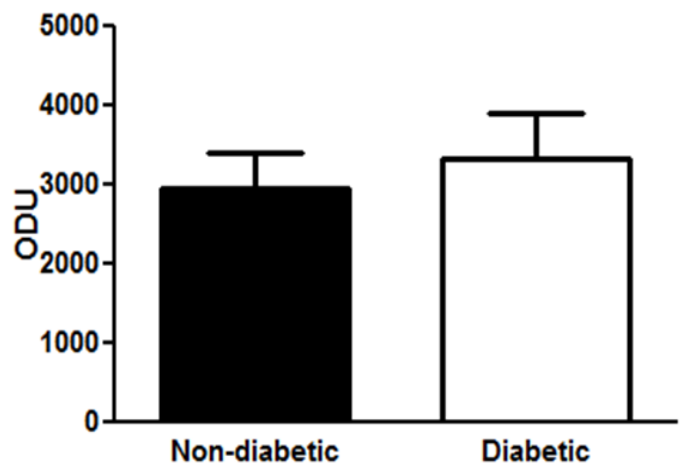


Figure 3.6 Electron transport chain complex I expression. Cardiac mitochondrial subpopulations were isolated and subjected to BN-PAGE to measure complex I protein content. A component of ETC complex I was used to measure the protein content of complex I. The BN-PAGE blots are illustrated in the non-diabetic and diabetic SSM (A) and IFM (C). The optical density was calculated in non-diabetic and diabetic SSM (B) and IFM (D). Values are mean \pm SEM. Closed bars represent non-diabetic patients and open bars represent diabetic patients. ${ }^{*} \mathrm{P} \leq 0.05$ SSM non-diabetic vs. SSM diabetic; $N$ $=4$ non-diabetics and 4 diabetics. 
Figure 3.7

A. SSM Complex IV Blue Native

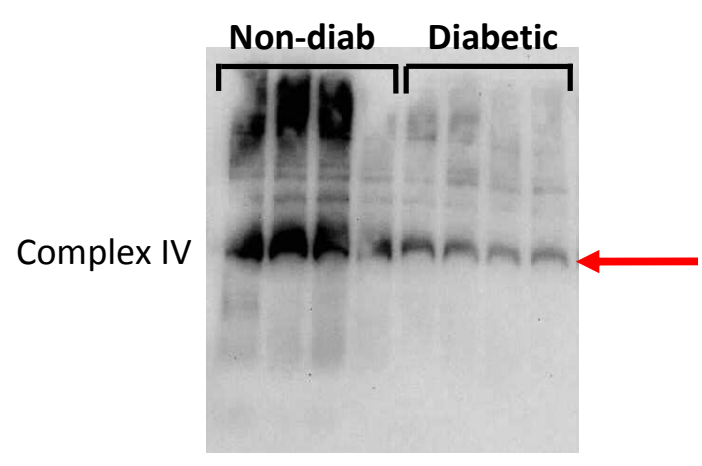

C. IFM Complex IV Blue Native

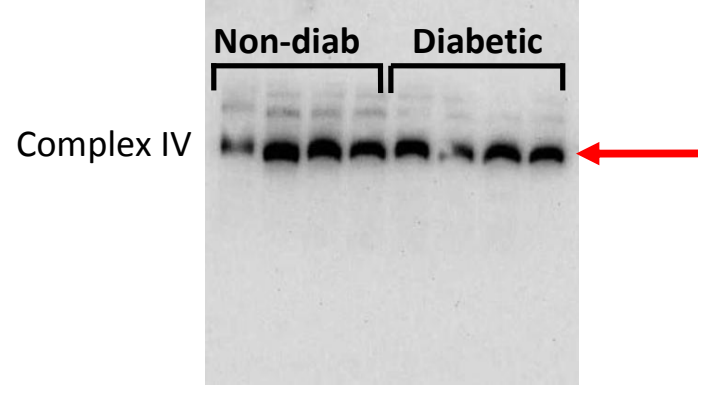

B. SSM: Complex IV

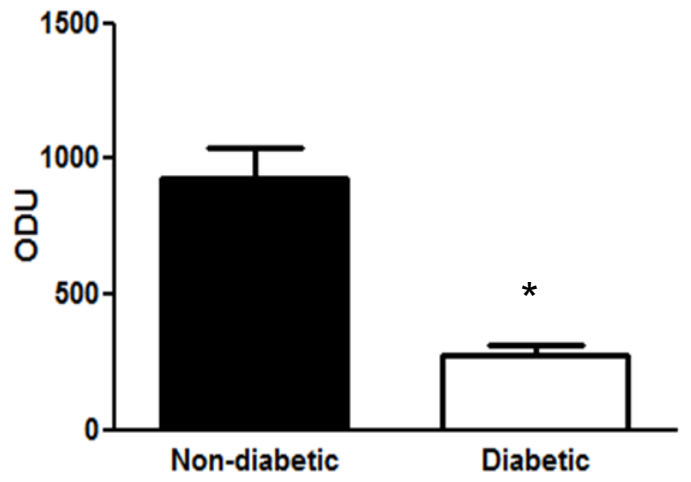

D.

IFM: Complex IV

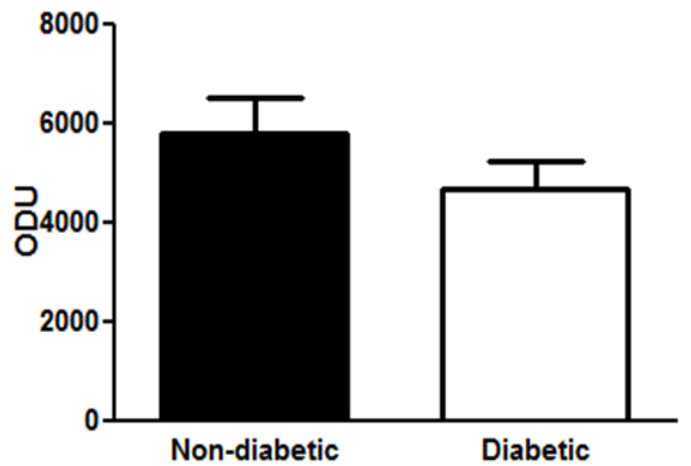


Figure 3.7 Electron transport chain complex IV expression. Cardiac mitochondrial subpopulations were isolated and subjected to BN-PAGE to measure complex I and complex IV protein content. A component of ETC complex IV was used to measure the protein content of complex IV. The BN-PAGE blots are illustrated in the non-diabetic and diabetic SSM (A) and IFM (C). The optical density was calculated in non-diabetic and diabetic SSM (B) and IFM (D). Values are mean \pm SEM. Closed bars represent non-diabetic patients and open bars represent diabetic patients. ${ }^{*} \mathrm{P} \leq 0.05 \mathrm{SSM}$ nondiabetic vs. SSM diabetic; $N=4$ non-diabetics and 4 diabetics. 
Figure 3.8

A.

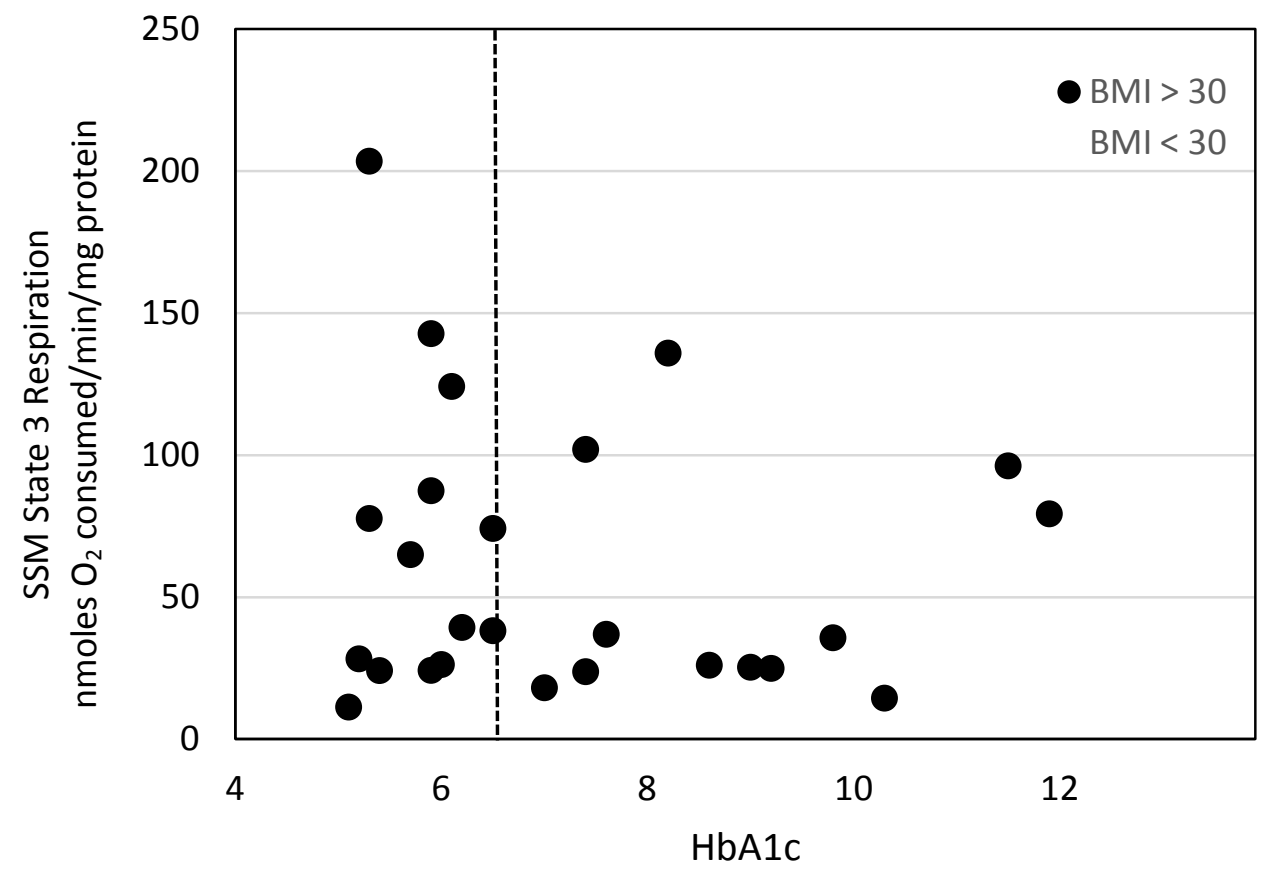

B.

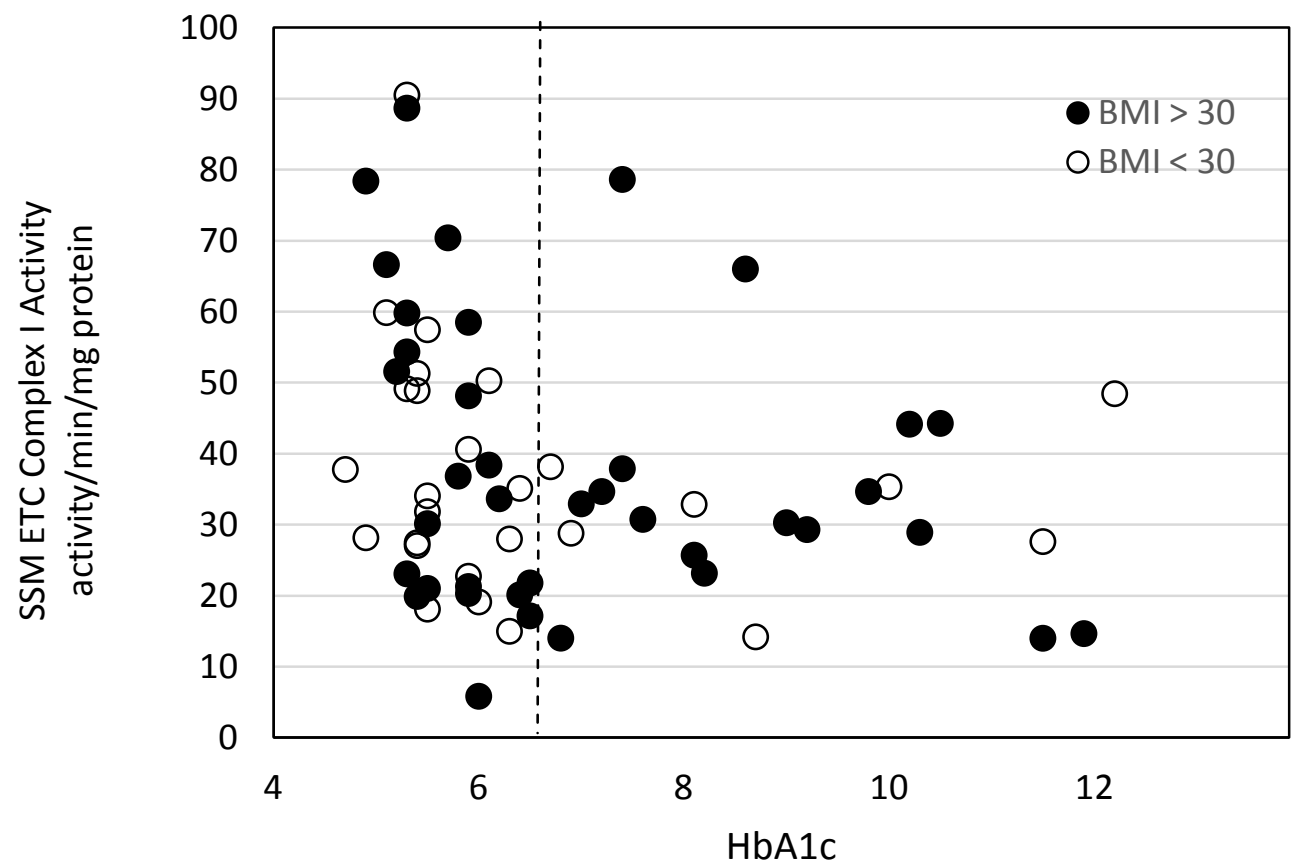


Figure 3.8 Linear spline illustration for state 3 mitochondrial respiration and ETC complex I activity. Linear spline models for HbA1c and SSM state 3 mitochondrial respiration and SSM ETC complex I activity, respectively, with a break-point at an $\mathrm{HbA} 1 \mathrm{c}$ levelg equal to $6.5 \%$ (dashed line). (A) Same linear spline model as Figure 3.3A and $(B)$ is the same graph as Figure $3.5 \mathrm{~A}$, with dots representing each patient with an observed HbA1c level. Open dots represent patients whose BMI is less than $30 \mathrm{~kg} / \mathrm{m}^{2}$, whereas closed dots represent patients whose BMI is greater than $30 \mathrm{~kg} / \mathrm{m}^{2}$. Units are nmoles $\mathrm{O}_{2}$ consumed/min/mg of protein for mitochondrial respiration and activity $/ \mathrm{min} / \mathrm{mg}$ of protein for ETC complex I activity. 
Table 3.1

Non-diabetic Diabetic

\begin{tabular}{|c|c|c|c|}
\hline \multirow[t]{3}{*}{ Demographics } & Age (years) & $60.0 \pm 1.9$ & $61.2 \pm 1.8$ \\
\hline & $\operatorname{Sex}(M / F)$ & $38 / 9$ & $22 / 12$ \\
\hline & Race & Caucasian & Caucasian \\
\hline \multirow[t]{6}{*}{ Characteristics } & BMI & $29.8 \pm 0.8$ & $32.8 \pm 1.1^{*}$ \\
\hline & HbA1c $\quad(n=37,33)$ & $5.5 \pm 0.1$ & $8.5 \pm 0.4^{*}$ \\
\hline & Avg. $B G_{(n=37,33)}$ & $111.8 \pm 2.4$ & $199.2 \pm 12.2^{*}$ \\
\hline & EF & $52.8 \pm 2.1$ & $49.2 \pm 2.7$ \\
\hline & Hypertension $(n=46,34)$ & $78.3 \%$ & $85.3 \%$ \\
\hline & $\mathrm{CAD}_{(\mathrm{n}=47,34)}$ & $83.0 \%$ & $91.2 \%$ \\
\hline \multirow[t]{6}{*}{ Medications } & Insulin & $0 / 47$ & $12 / 34$ \\
\hline & Metformin & $0 / 47$ & $13 / 34$ \\
\hline & Sulfonylureas & $0 / 47$ & $6 / 34$ \\
\hline & $\beta$-blockers & $28 / 47$ & $26 / 34$ \\
\hline & ACE inhibitors & $15 / 47$ & $17 / 34$ \\
\hline & Statins & $27 / 47$ & $20 / 34$ \\
\hline
\end{tabular}


Table 3.1 Patient Demographics and Characteristics. * $P \leq 0.05$ Diabetic vs. Nondiabetic group. 
Table 3.2

\begin{tabular}{|l|c|c|c|c|c|}
\hline SSM & State 3 & State 4 & Complex I & Complex III & Complex IV \\
\hline $\begin{array}{l}\text { Non- } \\
\text { diabetic }\end{array}$ & $65.8 \pm 8.4$ & $4.1 \pm 0.9$ & $42.9 \pm 3.6$ & $24.6 \pm 4.9$ & $16.8 \pm 2.1$ \\
\hline Diabetic & $39.4 \pm 6.0^{\star}$ & $1.8 \pm 0.3^{\star}$ & $30.1 \pm 2.7^{\star}$ & $24.58 \pm 4.6$ & $11.0 \pm 1.3^{\star}$ \\
\hline IFM & & & & & \\
\hline $\begin{array}{l}\text { Non- } \\
\text { diabetic }\end{array}$ & $35.6 \pm 5.5$ & $3.4 \pm 1.0$ & $54.6 \pm 8.5$ & $51.8 \pm 11.5$ & $33.3 \pm 6.5$ \\
\hline Diabetic & $37.4 \pm 3.9$ & $2.3 \pm 0.6$ & $48.9 \pm 5.2$ & $50.6 \pm 10.7$ & $24.1 \pm 4.8$ \\
\hline
\end{tabular}


Table 3.2 Non-diabetic and Diabetic Patients with Both Coronary Artery Diseases and Hypertension. All patients have both coronary artery disease and hypertension. Diabetes mellitus is the only variable in this data set. Values are mean \pm SEM. Units for State 3 and State 4 are nmoles of oxygen consumed $/ \mathrm{min} / \mathrm{mg}$ of protein $(N=21$ Nondiabetics and 20 Diabetic patients). Units from complex activities are activity/min/mg of protein $\left(N=34\right.$ Non-diabetics and 27 Diabetic patients). ${ }^{*} \leq 0.05$ Diabetic vs. nondiabetic group. 
Table 3.3

\begin{tabular}{|l|c|c|c|c|c|}
\hline SSM & State 3 & State 4 & Complex I & Complex III & Complex IV \\
\hline $\begin{array}{l}\text { Non- } \\
\text { CAD }\end{array}$ & $82.5 \pm 10.7$ & $4.0 \pm 0.8$ & $41.8 \pm 3.3$ & $23.0 \pm 4.4$ & $16.3 \pm 1.9$ \\
\hline CAD & $84.8 \pm 27.6$ & $4.5 \pm 0.9$ & $47.2 \pm 10.1$ & $21.8 \pm 7.8$ & $20.7 \pm 3.3$ \\
\hline$\underline{\text { IFM }}$ & & & & & \\
\hline $\begin{array}{l}\text { Non- } \\
\text { CAD }\end{array}$ & $41.8 \pm 6.0$ & $3.3 \pm 0.8$ & $53.6 \pm 7.5$ & $50.3 \pm 10.1$ & $32.2 \pm 5.7$ \\
\hline CAD & $24.7 \pm 6.5$ & $3.6 \pm 0.9$ & $53.9 \pm 10.8$ & $18.4 \pm 3.1$ & $37.0 \pm 9.5$ \\
\hline
\end{tabular}




\section{Table 3.3 Non-Coronary Artery Diseases (CAD) and CAD Patients without}

Diabetes Mellitus. None of the patients have diabetes mellitus or hypertension. Coronary artery disease is the only variable in this data set. Values are mean \pm SEM. Units for State 3 and State 4 are nmoles of oxygen consumed $/ \mathrm{min} / \mathrm{mg}$ of protein $(N=28$ Non-CAD and 4 CAD patients). Units from complex activities are activity/min/mg of protein ( $N=42$ Non-CAD and 8 CAD patients). 
Table 3.4

\begin{tabular}{|l|c|c|c|c|c|}
\hline SSM & State 3 & State 4 & Complex I & Complex III & Complex IV \\
\hline Non-HT & $74 \pm 10.8$ & $4.1 \pm 0.9$ & $42.8 \pm 3.6$ & $24.5 \pm 4.7$ & $17.4 \pm 2.1$ \\
\hline HT & $110.5 \pm 20.4$ & $3.8 \pm 0.8$ & $42.5 \pm 7.2$ & $17.1 \pm 5.4$ & $16.0 \pm 2.7$ \\
\hline IFM & & & & & \\
\hline Non-HT & $34.7 \pm 5.4$ & $3.3 \pm 0.9$ & $54.7 \pm 8.2$ & $50.6 \pm 11.2$ & $34.2 \pm 6.4$ \\
\hline HT & $54.6 \pm 13.6$ & $3.3 \pm 0.7$ & $50.1 \pm 7.3$ & $29.1 \pm 7.3$ & $29.0 \pm 6.4$ \\
\hline
\end{tabular}


Table 3.4 Non-Hypertensive (HT) and Hypertensive Patients without Diabetes Mellitus. None of the patients have diabetes mellitus or coronary artery disease. Hypertension is the only variable in this data set. Values are mean \pm SEM. Units for State 3 and State 4 are nmoles of oxygen consumed/min/mg of protein ( $N=25$ Nonhypertensive (Non-HT) and 7 Hypertensive (HT)). Units from complex activities are activity/min/mg of protein ( $N=40$ Non-hypertensive and 10 Hypertensive patients). 


\section{Chapter 4:}

General Discussion 


\section{GENERAL DISCUSSION}

The overall objective of this dissertation was to evaluate the impact of both type 1 and type 2 diabetes mellitus on the IMM. Specifically, we wanted (1) to determine how the cardiolipin biosynthesis pathway and cardiolipin-protein interactions were impacted by type 1 diabetes mellitus; (2) to determine the therapeutic value of mitochondrial Grp75 overexpression on mitochondrial protein import during a type 1 diabetic insult; and (3) to examine how mitochondrial subpopulations in the human heart were affected by type 2 diabetes mellitus. The long term goal was to understand the importance of IMM preservation during diabetes mellitus and to translate rodent mitochondrial dysfunction that occurs during diabetic cardiomyopathy to the human diabetic heart. The central hypothesis of this dissertation was that the mitochondrion is negatively impacted by diabetes mellitus, leading to deleterious effects to the IMM and compromising the proteins associated with cardiolipin and protein import, which will translate to the diabetic human heart. The rationale for the research conducted for this dissertation is based on the importance of preserving the IMM structure and processes within to alleviate mitochondrial dysfunction associated with the diabetic heart, as well as understanding the effect of diabetes mellitus on human cardiac mitochondrial subpopulations.

Cardiovascular complications, such as diabetic cardiomyopathy, are the leading cause of morbidity and mortality in patients with diabetes mellitus $(13,15,25)$. Multiple studies have linked mitochondrial dysfunction with diabetic cardiomyopathy $(3,9,10$, 
$23,30,31$ ). Mitochondrial studies are complex in the fact that two distinct mitochondrial subpopulations reside in the heart (Figure 1.1). These two spatially distinct pools of mitochondria respond differently to pathological insults, such as diabetes mellitus (7-9, $18,20,24)$. We have previously reported mitochondrial dysfunction in the type 1 diabetic mouse heart, specifically in IFM, as evidenced by decreased mitochondrial respiration, ETC complexes I, III, IV, and V activities, as well as protein import $(3,9)$. Along with increased ROS production, lipid peroxidation and nitrotyrosine residues were significantly increased (9). Our laboratory has also reported detriments to the IMM and subsequent mitochondrial dysfunction, specifically in SSM of a type $2 \mathrm{db} / \mathrm{db}$ mouse model $(8,24)$, as evidenced by decreased mitochondrial respiration and decreased Grp75 protein content. We were the first to evaluate the impact of type 2 diabetes mellitus on mitochondrial subpopulations in human atrial tissue from which the results previously reported from the $\mathrm{db} / \mathrm{db}$ mouse heart translated into the type 2 diabetic human model, suggesting that mitochondria function from type 2 diabetic human heart displays similar mitochondrial dysfunctional profiles with SSM primarily impacted.

Literature suggests that ROS production is the underlying mechanism contributing to the mitochondrial dysfunction associated with diabetes mellitus $(6,14)$. The main source of ROS production is the IMM, making this membrane more susceptible to oxidative damage. The IMM is composed of a specific lipid environment that houses many of the critical mitochondrial processes that are required for mitochondria to function properly (Figure 1.2), such as oxidative phosphorylation and protein import machinery. Our laboratory has reported proteomic alterations in the IFM during a type 1 diabetic insult and in the SSM during a type 2 diabetic insult $(3,8)$. 
Figure $4.1 \mathrm{~A}$ represents the mitochondrial proteome and the abundance of proteins present in each membrane of the mitochondrion. Of the proteins altered during a type 1 diabetic insult, 51\% reside in the IMM (Figure 4.1B). Similarly, of the proteins altered during a type 2 diabetic insult, 50\% reside in the IMM (Figure 4.1C). By comparing the pie charts between the different disease states versus control, one can recognize that the IMM is most affected by diabetes mellitus (17).

\section{A. Mitochondrial Proteome}

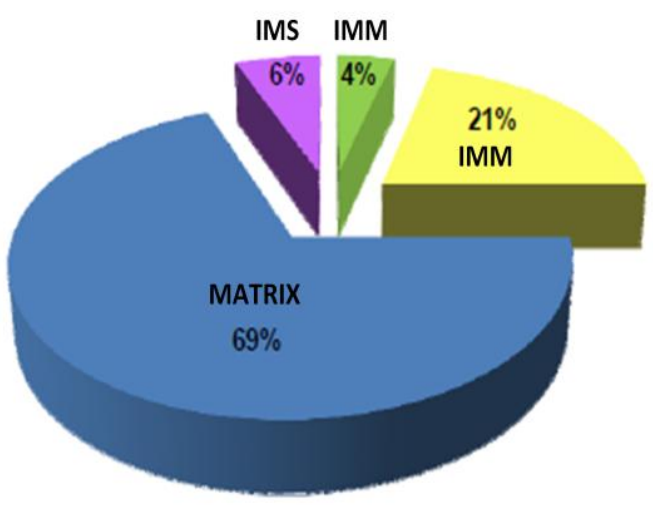

B. Type 1 Diabetic IFM

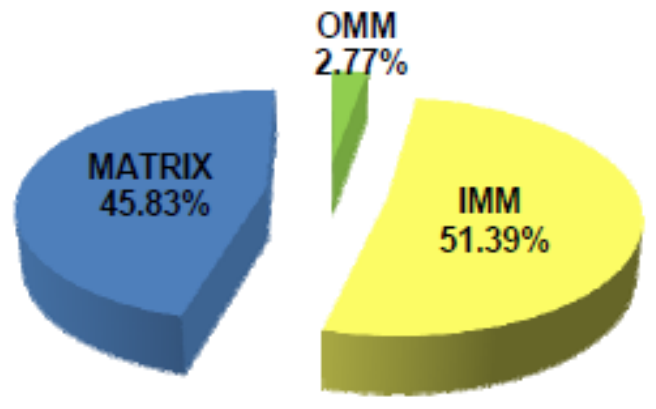

\section{Mitochondrial compartments:}

- $\mathrm{OMM}=$ outer mitochondrial membrane

- $\mathrm{IMM}=$ inner mitochondrial membrane

- $\mathrm{IMS}=$ inner mitochondrial space

- Matrix

\section{Type 2 Diabetic SSM}

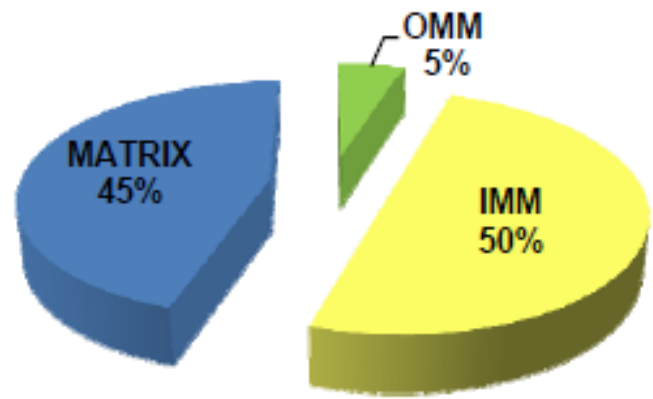

Figure 4.1. Mitochondrial proteome during different diabetic states. (A) Pie chart representing the percentage of proteins present in each membrane of mitochondria in a control setting. (B) Pie chart representing the percentage of proteins altered during a 
type 1 diabetic insult in the IFM. (C) Pie chart representing the percentage of proteins altered during a type 2 diabetic insult in the SSM (17).

Cardiolipin is a key phospholipid that is required for the stability of the IMM (27), as well as for proper functioning of many IMM proteins (Figure 1.3), including the oxidative phosphorylation machinery, cytochrome $c$ attachment and protein import machinery $(12,19,22,28,32)$. Tetralinoleic cardiolipin is the most dominant form of cardiolipin in the heart; however, other forms exist in different tissues and during different pathologies $(1,27)$. Our laboratory and others reported a decrease in total cardiolipin and tetralinoleic cardiolipin during a type 1 diabetic insult (Chapter 2) (16), specifically in IFM (9). Preliminary data generated from type 2 diabetic human atrial tissue utilizing high performance thin layer chromatography also revealed lower total cardiolipin content in the diabetic SSM (not significant) (Figure 6.5).

Although both type 1 and type 2 diabetes mellitus are a consequence of a hyperglycemic environment, the manifestation and phenotypes between the diseases differ. For example, chapters 2 and 6 reported decreased cardiolipin content in both IFM (type 1) and SSM (type 2), which could be caused by different pathways depending on the type of diabetes mellitus. During type 2 diabetes mellitus, fatty acid oxidation and oxidative stress precede the hyperglycemic environment (5), from which circulating fatty acids, as well as triglyceride levels, are increased (33). Because SSM are located just beneath the sarcolemma, this population is affected to a greater extent than IFM during type 2 diabetes mellitus. Supporting this statement, proteins involved in fatty acid 
oxidation were reported to be increased to a greater extent in type 2 diabetic SSM, compared to IFM, resulting in an enhanced lipid environment (8). Literature has also stated that individuals with type 2 diabetes mellitus tend to be more obese and were subjected to myocardial lipotoxicity (4); therefore, cardiolipin content could be decreased in type 2 diabetic SSM due to the enhanced oxidative environment and increased lipotoxicity.

During type 1 diabetes mellitus, the hyperglycemic environment causes an enhanced oxidative environment, from which post-translational modification analyses revealed a higher number of oxidations in type 1 diabetic IFM, compared to type 2 diabetic SSM. This enhanced oxidative environment is a result of increased ROS production that damages important proteins and subsequent processes that are required for proper mitochondrial function. Along with a higher membrane potential, IFM have higher respiration and protein import rates compared to SSM. Because of these characteristics, type 1 diabetic IFM produce higher levels of ROS; and consequently, are impacted to a greater extent than SSM. Although the reported decrease in cardiolipin content in type 1 diabetic IFM could also be caused by the oxidative environment, data reported in chapter 2 indicated that an important constituent required for cardiolipin biosynthesis was significantly decreased; therefore, the enhanced oxidative environment could be the underlying mechanism contributing to the defective biosynthesis of cardiolipin. To identify the mechanism contributing to decreased cardiolipin content, we first evaluated the biosynthetic pathway of cardiolipin during a type 1 diabetic insult (Chapter 2). 
The cardiolipin biosynthetic pathway is a multi-step process that requires many enzymatic reactions to occur in order for the mature form of cardiolipin to be produced (Figures 1.4 and 2.1) $(26,27)$. We hypothesized that at least one of the reactions involved in this pathway was affected by type 1 diabetes mellitus. The mRNA and protein levels of the enzymes directly responsible for the synthesis of cardiolipin were analyzed and results indicated that none of the mRNA levels were altered in the diabetic heart; and interestingly, only one protein was significantly decreased in the type 1 diabetic IFM - CRLS (Table 2.1 and Figure 2.2). Because the protein content of CRLS was decreased, we next analyzed its activity and found that CRLS activity was significantly decreased in the type 1 diabetic IFM (Figure 2.3). These results taken together suggest that the main contributors to the loss of cardiolipin content during both type 1 and type 2 diabetes mellitus is decreased CRLS content and activity.

Housed in the IMM, the oxidative phosphorylation machinery requires an association with cardiolipin to function properly (Figure 1.2) $(11,12,27,29)$. In both the type 1 and type 2 diabetic mouse model, data produced from our laboratory indicated decreased ETC complex activity for complexes I, III, IV and V in the diabetic IFM and diabetic SSM, respectively (Figure 2.4) $(8,9)$. Similarly in the type 2 diabetic human heart model, ETC complex I and IV activities, as well as the protein expression of the aforementioned complexes were decreased (Figures 3.4, 3.6 and 3.7). Data reported in chapter 2 revealed decreased associations between cardiolipin and ATP synthase subunits in the presence of a type 1 diabetic insult (Figure 2.6); therefore, one potential 
mechanism contributing to the decreased ETC complex activities may be the decrease in cardiolipin content.

The dysfunctional ETC complexes can further negatively impact the IMM through different pathways. First, the IMM is the primary source of ROS production, specifically at ETC complex I and complex III (21); therefore, defective ETC complexes I and III could cause an increase in ROS, which would further inhibit proper mitochondrial function. For example, an increase in ROS can enhance the oxidative environment of mitochondria causing stress to the organelle and its functional processes. Also, an increase in ROS can target the double bonds composing tetralinoleic cardiolipin (Figure 1.3). This alteration in cardiolipin composition can negatively impact the interactions between the lipid environment and important associated proteins, rendering IMM proteins, such as ETC complexes, dysfunctional. With a lower cardiolipin content already present during a diabetic insult, alterations to the remaining lipid environment could severely damage cardiac mitochondria. Secondly, if the ETC complexes do not function properly during a diabetic insult, ATP production may decrease, causing mitochondrial dysfunction and ultimately, cardiac contractile dysfunction. Taken all together, the results suggest possible mechanisms underlying the decreased ETC complex activities, all of which are deleterious effects of diabetes mellitus to the IMM.

Processes housed within the IMM also require a proper membrane potential which is generated by the IMM. Cardiolipin plays a role in membrane stability (27); and therefore, in the maintenance of the membrane potential. Because the content of 
cardiolipin is decreased during a diabetic insult, the membrane potential becomes unstable. Using mouse models, we have previously reported decreased membrane potential in type 1 diabetic IFM (9), as well as in type 2 diabetic SSM (8). This decrease in membrane potential negatively impacts the processes that are located within the IMM, such as oxidative phosphorylation and protein import.

Glucose - mediated and fatty acid - mediated respiration were both decreased in SSM of type 2 diabetic human atrial tissue, with no change observed in IFM (Chapter 3). These data are in partial agreement with those data that we reported previously in the $\mathrm{db} / \mathrm{db}$ mouse heart (8). In a type 1 diabetic mouse model, state 3 mitochondrial respiration was also reported to be decreased specifically in IFM (9). Because mitochondrial respiration relies on a membrane potential, this decrease observed in mitochondrial respiration could be attributed in part to the decreased membrane potential in type 1 diabetic IFM and type 2 diabetic SSM.

As depicted in Figure 1.2, the protein import machinery also resides in the IMM and is known to interact with cardiolipin $(19,32)$. Both protein import activity and a key constituent involved in the protein import process, Grp75, have been reported to be decreased during type 1 diabetes mellitus (3). In type 2 diabetic human atrial tissue, preliminary data examining protein import revealed decreased protein import rates in diabetic SSM compared to a non-diabetic heart, with no effect on IFM (Figure 6.4). Grp75 protein content was also decreased in human type 2 diabetic SSM (Figure 6.3). The influence of diabetes on protein import and Grp75 protein levels could be altered 
due to a number of reasons. First, cardiolipin is known to be associated with the protein import machinery $(19,32)$ and the decrease in cardiolipin content could subsequently contribute to the loss in protein import activity (Chapter 2). Secondly, a loss of Grp75 protein content could either be a result of or a mechanism contributing to the decreased protein import (Chapter 6) $(3,8)$. Because decreased protein import is a critical contributor to diabetic-associated mitochondrial dysfunction, a novel transgenic mouse line overexpressing Grp75 was generated in our laboratory (Figure 6.1).

To ensure that the Grp75 overexpressing transgenic mouse was in fact overexpressing Grp75, we co-incubated mitochondrial subpopulations from a control and a transgenic mouse with MitoTracker Deep Red 633, which specifically stain respiring mitochondria, along with a Grp75-PE conjugated antibody. We subjected the co-stained mitochondria to flow cytometry, which was able to detect the mitochondria stained with both MitoTracker Deep Red 633 and Grp75-PE, since each dye used a different laser to emit fluorescence. Table 6.1 depicted the results of Grp75 overexpression between a control mouse and a transgenic mouse as measured by the mean fluorescence intensity, from which we concluded that the Grp75 mouse overexpressed Grp75 compared to its control counterpart.

An additional method to measure Grp75 overexpression would be to perform a Western blot; however, if Grp75 overexpression increased protein import, then the Western blot can no longer be loaded per mitochondrial protein content because the amount of protein between control and the transgenic mouse would more than likely not 
be equal. Therefore, the amount of intact mitochondria in each isolated sample could be measured by selectively staining for intact mitochondria and subjecting the mitochondria to flow cytometry $(9,34)$. A Western blot could then be loaded per intact mitochondria; however, the isolated mitochondrial sample would contain not only intact mitochondria, but also debris; therefore, without separating the debris, a Western blot would not truly depict the amount of Grp75 protein in the overexpressing transgenic mouse. Because of these limitations, analyzing the mean fluorescence intensity of co-stained mitochondrial sub-populations represented the most accurate overexpression measurement.

Once the overexpression of Grp75 was established, the therapeutic benefit of overexpressing GRP75 on mitochondrial function during a diabetic insult was evaluated. Mitochondrial respiration is decreased in IFM during a type 1 diabetic insult (9); however, in the presence of Grp75 overexpression, state 3 mitochondrial respiration was restored in diabetic IFM, as seen in Figure 6.2, with no effect on state 4 or SSM. Taken together, the preliminary data collected demonstrated that the overexpression of Grp75 has the ability to provide cardioprotection in the presence of diabetes mellitus. In order to fully determine the therapeutic advantage to overexpressing Grp75, cardiac functional analyses and further mitochondrial experimentation, including protein import, is warranted.

Table 4.1 lists the reported deleterious effects to mitochondrial subpopulation function observed during diabetes mellitus. Taken together, the mitochondrion is negatively impacted by diabetes mellitus regardless of the type of diabetes mellitus or 
the mitochondrial subpopulation affected. This negative impact leads to deleterious effects to the IMM and compromising the proteins associated with the lipid environment, specifically cardiolipin. Decreased protein import could be contributing to the loss of the CRLS protein, from which cardiolipin content are reduced. This reduction in cardiolipin content contributes to the dysfunctional processes housed within the IMM, including decreased oxidative phosphorylation, as well as further exacerbation of protein import activity. Genetic manipulation of Grp75 provided protection against diabetes-associated mitochondrial dysfunction, possibly by preserving the IMM through restored cardiolipin synthase protein levels and consequently, cardiolipin content. This restoration in cardiolipin content would preserve cardiolipin-IMM protein associations, as well as key processes required for proper mitochondrial function; however, further studies are warranted. The preliminary data gathered from evaluating the overexpression of Grp75 may lend insight to a possible therapeutic target to alleviate the detrimental effects of diabetes mellitus on mitochondrial function. Further, because an increase in the oxidative environment during type 1 diabetes mellitus has been previously reported (2), another possible beneficial treatment could be developed by targeting the antioxidant capacity of mitochondria and inhibiting the increased oxidative environment during type 1 diabetes mellitus. Potential therapeutic strategies are further discussed in Chapter 5. Nonetheless, the damage to the IMM signifies the importance of preserving the IMM by developing therapeutic strategies aimed at relieving mitochondrial dysfunction. 
Table 4.1. Mitochondrial dysfunction associated with type 1 and type 2 diabetes mellitus in mouse and human models.

\begin{tabular}{|c|c|c|c|c|c|c|}
\hline \multirow[b]{3}{*}{ State 3 Respiration (G:M) } & \multicolumn{2}{|c|}{$\begin{array}{c}\text { Type } 1 \text { diabetic } \\
\text { mouse (STZ) }\end{array}$} & \multicolumn{2}{|c|}{$\begin{array}{l}\text { Type } 2 \text { diabetic } \\
\text { mouse (db/db) }\end{array}$} & \multicolumn{2}{|c|}{$\begin{array}{c}\text { Type } 2 \text { diabetic } \\
\text { human }\end{array}$} \\
\hline & $\underline{\text { SSM }}$ & $\underline{I F M}$ & $\underline{\text { SSM }}$ & $\underline{\mathrm{IFM}}$ & SSM & $\underline{I F M}$ \\
\hline & $\overline{-}$ & $\overline{\downarrow^{\#}}$ & $\bar{\downarrow}$ & $\overline{-}$ & $\bar{\downarrow}$ & $\overline{-}$ \\
\hline State 4 Respiration (G:M) & - & N/A & - & - & $\downarrow$ & - \\
\hline State 3 Respiration (PC:M) & N/A & N/A & $\downarrow$ & - & $\downarrow$ & - \\
\hline State 4 Respiration (PC:M) & N/A & N/A & $\downarrow$ & - & $\downarrow$ & - \\
\hline ETC Complex I & - & $\downarrow$ & $\downarrow$ & - & $\downarrow$ & - \\
\hline ETC Complex III & - & $\downarrow$ & $\downarrow$ & - & - & - \\
\hline ETC Complex IV & - & $\downarrow$ & $\downarrow$ & - & $\downarrow$ & - \\
\hline ETC Complex V & - & $\downarrow$ & $\downarrow$ & - & - & - \\
\hline Cardiolipin content & - & $\downarrow$ & N/A & N/A & $\downarrow^{\$ *}$ & - \\
\hline Tetralinoleic cardiolipin content & - & $\downarrow$ & N/A & N/A & N/A & N/A \\
\hline Cardiolipin-key protein associations & - & $\downarrow$ & N/A & N/A & N/A & N/A \\
\hline Protein import & - & $\downarrow$ & N/A & N/A & $\downarrow^{*}$ & $-*$ \\
\hline Grp75 content & - & $\downarrow$ & $\downarrow$ & - & $\downarrow$ & - \\
\hline
\end{tabular}

In conclusion, our data exemplifies the importance of recognizing that most of the processes and subsequent proteins affected during both type 1 and type 2 diabetes mellitus are housed within the IMM, suggesting that the IMM is targeted by diabetes mellitus. Further, the data collected from examining mitochondrial dysfunction during a type 1 and type 2 diabetic insult provides evidence for the importance of examining the two mitochondrial subpopulations separately because the effects of each type of diabetes mellitus impacts the mitochondrial subpopulations oppositely. The results of this dissertation are in agreement with the hypothesis in that the mitochondrion is negatively impacted by diabetes mellitus, leading to deleterious effects to the IMM and compromising the proteins associated with cardiolipin and protein import, which 
translates to the diabetic human heart. The results of the studies in this dissertation establish the importance preserving the IMM and those processes contained within to alleviate mitochondrial dysfunction associated with the diabetic heart. 


\subsection{References}

1. Acehan D, Vaz F, Houtkooper RH, James J, Moore V, Tokunaga C, Kulik W, Wansapura J, Toth MJ, Strauss A, and Khuchua Z. Cardiac and skeletal muscle defects in a mouse model of human Barth syndrome. J Biol Chem 286: 899-908, 2011.

2. Baseler WA, Dabkowski ER, Jagannathan R, Thapa D, Nichols CE, Shepherd DL, Croston TL, Powell M, Razunguzwa TT, Lewis SE, Schnell DM, and Hollander JM. Reversal of mitochondrial proteomic loss in Type 1 diabetic heart with overexpression of phospholipid hydroperoxide glutathione peroxidase. Am J Physiol Regul Integr Comp Physiol 304: R553-565, 2013.

3. Baseler WA, Dabkowski ER, Williamson CL, Croston TL, Thapa D, Powell MJ, Razunguzwa TT, and Hollander JM. Proteomic alterations of distinct mitochondrial subpopulations in the type 1 diabetic heart: contribution of protein import dysfunction. Am J Physiol Regul Integr Comp Physiol 300: R186-200, 2011.

4. Boudina S and Abel ED. Diabetic cardiomyopathy, causes and effects. Rev Endocr Metab Disord 11: 31-39, 2010.

5. Boudina S, Sena S, Theobald H, Sheng X, Wright JJ, Hu XX, Aziz S, Johnson Jl, Bugger H, Zaha VG, and Abel ED. Mitochondrial energetics in the heart in obesity-related diabetes: direct evidence for increased uncoupled respiration and activation of uncoupling proteins. Diabetes 56: 2457-2466, 2007.

6. Brownlee M. The pathobiology of diabetic complications: a unifying mechanism. Diabetes 54: 1615-1625, 2005. 
7. Chen Q, Moghaddas S, Hoppel CL, and Lesnefsky EJ. Ischemic defects in the electron transport chain increase the production of reactive oxygen species from isolated rat heart mitochondria. Am J Physiol Cell Physiol 294: C460-466, 2008.

8. Dabkowski ER, Baseler WA, Williamson CL, Powell M, Razunguzwa TT, Frisbee JC, and Hollander JM. Mitochondrial dysfunction in the type 2 diabetic heart is associated with alterations in spatially distinct mitochondrial proteomes. Am J Physiol Heart Circ Physiol 299: H529-540, 2010.

9. Dabkowski ER, Williamson CL, Bukowski VC, Chapman RS, Leonard SS, Peer CJ, Callery PS, and Hollander JM. Diabetic cardiomyopathy-associated dysfunction in spatially distinct mitochondrial subpopulations. Am J Physiol Heart Circ Physiol 296: H359-369, 2009.

10. Devereux RB, Roman MJ, Paranicas M, OÂ'Grady MJ, Lee ET, Welty TK, Fabsitz RR, Robbins D, Rhoades ER, and Howard BV. Impact of Diabetes on Cardiac Structure and Function : The Strong Heart Study. Circulation 101: 2271-2276, 2000.

11. Eble KS, Coleman WB, Hantgan RR, and Cunningham CC. Tightly associated cardiolipin in the bovine heart mitochondrial ATP synthase as analyzed by 31P nuclear magnetic resonance spectroscopy. J Biol Chem 265: 19434-19440, 1990.

12. Fry $\mathbf{M}$ and Green DE. Cardiolipin requirement for electron transfer in complex I and III of the mitochondrial respiratory chain. Journal of Biological Chemistry 256: 18741880, 1981. 
13. Garcia MJ, McNamara PM, Gordon T, and Kannel WB. Morbidity and mortality in diabetics in the Framingham population. Sixteen year follow-up study. Diabetes 23: 105-111, 1974.

14. Green K, Brand MD, and Murphy MP. Prevention of mitochondrial oxidative damage as a therapeutic strategy in diabetes. Diabetes 53 Suppl 1: S110-118, 2004.

15. Grundy SM, Benjamin IJ, Burke GL, Chait A, Eckel RH, Howard BV, Mitch W, Smith SC, Jr., and Sowers JR. Diabetes and cardiovascular disease: a statement for healthcare professionals from the American Heart Association. Circulation 100: 1134-1146, 1999.

16. Han X, Yang J, Cheng H, Yang K, Abendschein DR, and Gross RW. Shotgun lipidomics identifies cardiolipin depletion in diabetic myocardium linking altered substrate utilization with mitochondrial dysfunction. Biochemistry 44: 16684-16694, 2005.

17. Hollander JM, Baseler WA, and Dabkowski ER. Proteomic remodeling of mitochondria in heart failure. Congest Heart Fail 17: 262-268, 2011.

18. Hoppel CL, Moghaddas S, and Lesnefsky EJ. Interfibrillar cardiac mitochondrial comples III defects in the aging rat heart. Biogerontology 3: 41-44, 2002.

19. Houtkooper R and Vaz F. Cardiolipin, the heart of mitochondrial metabolism. Cellular and Molecular Life Sciences 65: 2493-2506, 2008.

20. Lesnefsky EJ, Chen Q, Slabe TJ, Stoll MSK, Minkler PE, Hassan MO, Tandler B, and Hoppel CL. Ischemia, rather than reperfusion, inhibits respiration through cytochrome oxidase in the isolated, perfused rabbit heart: role of cardiolipin. Am J Physiol Heart Circ Physiol 287: H258-267, 2004. 
21. Orrenius S, Gogvadze V, and Zhivotovsky B. Mitochondrial oxidative stress: implications for cell death. Annu Rev Pharmacol Toxicol 47: 143-183, 2007.

22. Petrosillo G, Ruggiero FM, and Paradies G. Role of reactive oxygen species and cardiolipin in the release of cytochrome c from mitochondria. FASEB J 17: 22022208, 2003.

23. Regan TJ, Lyons MM, Ahmed SS, Levinson GE, Oldewurtel HA, Ahmad MR, and Haider B. Evidence for cardiomyopathy in familial diabetes mellitus. J Clin Invest 60: 884-899, 1977.

24. Ritov VB, Menshikova EV, He J, Ferrell RE, Goodpaster BH, and Kelley DE. Deficiency of subsarcolemmal mitochondria in obesity and type 2 diabetes. Diabetes 54 : 8-14, 2005.

25. Rubler S, Dlugash J, Yuceoglu YZ, Kumral T, Branwood AW, and Grishman A. New type of cardiomyopathy associated with diabetic glomerulosclerosis. Am J Cardiol 30: 595-602, 1972.

26. Saini-Chohan HK, Holmes MG, Chicco AJ, Taylor WA, Moore RL, McCune SA, Hickson-Bick DL, Hatch GM, and Sparagna GC. Cardiolipin biosynthesis and remodeling enzymes are altered during development of heart failure. Journal of Lipid Research 50: 1600-1608, 2009.

27. Schlame M, Rua D, and Greenberg ML. The biosynthesis and functional role of cardiolipin. Prog Lipid Res 39: 257-288, 2000.

28. Sedlak E and Robinson NC. Phospholipase A2 Digestion of Cardiolipin Bound to Bovine Cytochrome c Oxidase Alters Both Activity and Quaternary Structureâ€ Biochemistry 38: 14966-14972, 1999. 
29. Sedlak E and Robinson NC. Phospholipase A(2) digestion of cardiolipin bound to bovine cytochrome c oxidase alters both activity and quaternary structure. Biochemistry 38: 14966-14972, 1999.

30. Severson DL. Diabetic cardiomyopathy: recent evidence from mouse models of type 1 and type 2 diabetes. Can J Physiol Pharmacol 82: 813-823, 2004.

31. Tomita M, Mukae S, Geshi E, Umetsu K, Nakatani M, and Katagiri T. Mitochondrial respiratory impairment in streptozotocin-induced diabetic rat heart. Jpn Circ J 60: 673-682, 1996.

32. Torok Z, Demel RA, Leenhouts JM, and de Kruijff B. Presequence-mediated intermembrane contact formation and lipid flow. A model membrane study. Biochemistry 33: 5589-5594, 1994.

33. Wende AR and Abel ED. Lipotoxicity in the heart. Biochimica et Biophysica Acta (BBA) - Molecular and Cell Biology of Lipids 1801: 311-319, 2010.

34. Williamson CL, Dabkowski ER, Baseler WA, Croston TL, Alway SE, and Hollander JM. Enhanced apoptotic propensity in diabetic cardiac mitochondria: influence of subcellular spatial location. Am J Physiol Heart Circ Physiol 298: H633-642, 2010. 


\section{Chapter 5:}

\section{Future Directions}




\section{FUTURE DIRECTIONS}

Research has established an association between mitochondrial dysfunction and the type 2 diabetic heart (2-4); however, the lipid environment, lipid-protein associations, and specific processes contained within have not yet been examined in diabetic mitochondrial subpopulations. Future studies examining the IMM phospholipid environment in a mouse model of type 2 diabetes mellitus, specifically cardiolipin and cardiolipin remodeling, would advance the understanding of the environment and the protein interactions within, which are required for proper mitochondrial function. Preliminary data collected for the studies included in this dissertation determined lower cardiolipin content in type 2 diabetic SSM from human atrial tissue, which was gathered from high performance thin layer chromatography and iodine vapor staining. A more accurate method employing mass spectrometry would more efficiently measure the levels of total cardiolipin content, as well as the levels of different cardiolipin species. Data reported in chapter 2 revealed decreased interactions between cardiolipin and ATP synthase subunits in a type 1 diabetic mouse model, specifically in IFM. These interactions could also be measured during a type 2 diabetic insult, as well as the interactions between cardiolipin and other proteins housed in the IMM. Together, these results could potentially lend insight as to why some of the proteomic alterations observed during a type 2 diabetic insult (4) may be occurring.

After revealing the negative impact of type 2 diabetes mellitus on the SSM in

human atrial tissue (Chapter 3), alterations in the mitochondrial proteome should be 
further examined. Chapter 3 included data that reported decreased ETC complex I and IV activities in type 2 diabetic SSM. The underlying mechanism causing this decrease in ETC complex I and IV activities was determined to be decreased protein expression of ETC complex I and IV. Although this measurement included the ETC complex as a whole unit, individual subunits are also more than likely affected by a type 2 diabetic insult; therefore, this finding warrants further experimentation examining alterations in the mitochondrial proteomic makeup during type 2 diabetes mellitus.

Preserving the IMM can be accomplished by focusing on different proteins or processes located within the IMM. For example, during diabetes mellitus, mitochondrial membrane potential is decreased and in conjunction with a disruption of the lipid environment, specifically cardiolipin, a preservation strategy targeting the structure and integrity of the IMM could prove to be beneficial in protecting mitochondrial function. This could be through genetic manipulation of structural proteins that are altered in the presence of diabetes mellitus. In order to conserve the IMM integrity, another strategy could target the phospholipid environment.

Total cardiolipin content was lower in SSM of type 2 diabetic human atrial tissue (Chapter 3), and significantly decreased in IFM of type 1 diabetic mouse hearts (Chapter 2) along with tetralinoleic cardiolipin (5). The data in chapter 2 also revealed decreased CRLS protein content and activity, which suggested that the decrease in cardiolipin was due to deficient cardiolipin biosynthesis at the level of CRLS. In order to 
restore cardiolipin content, our laboratory created a genetically modified mouse model that overexpressed CRLS (Figure 5.1).

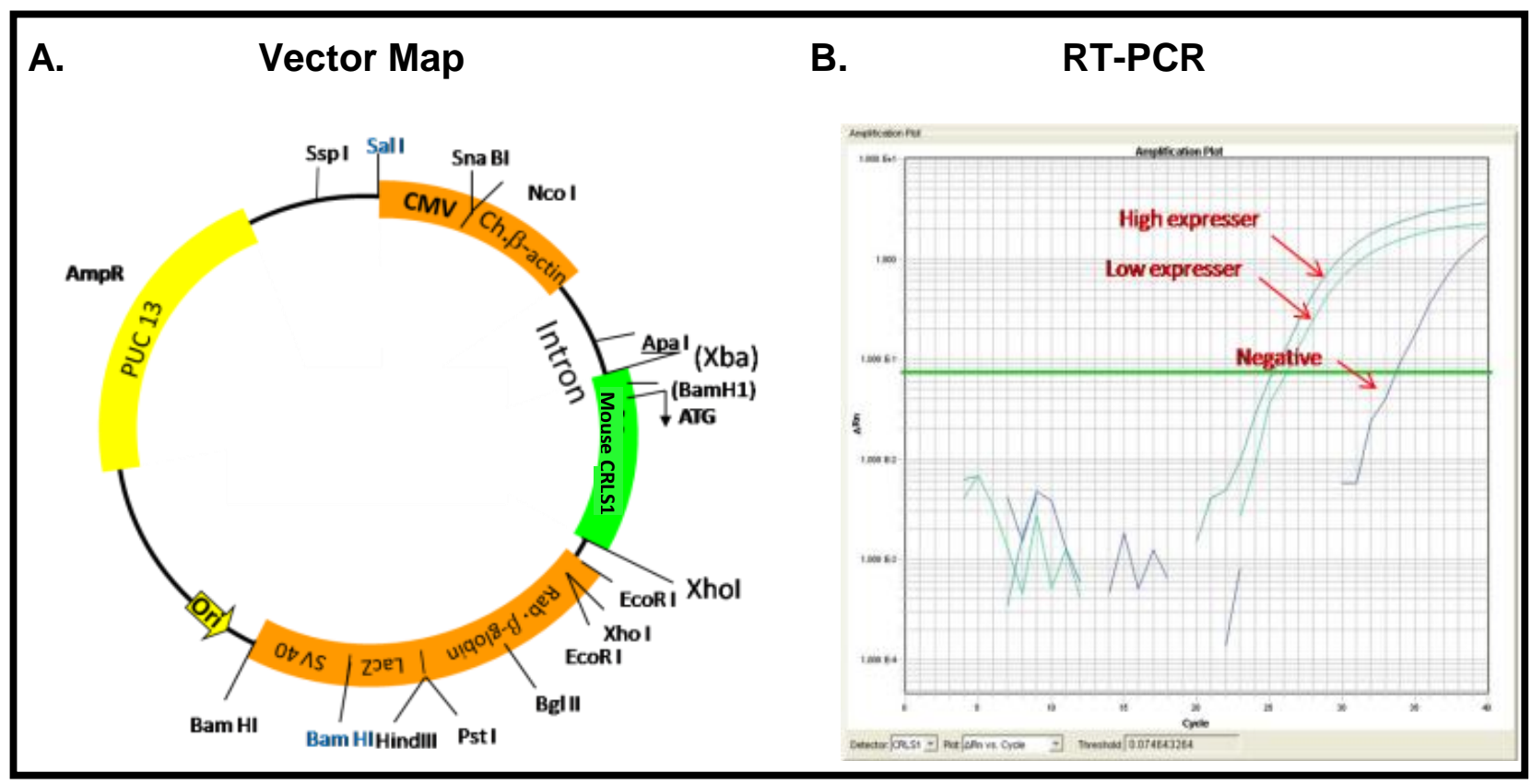

Figure 5.1. CRLS pCAGGS vector map. (A) Map of CLS transgenic construct using pCAGGS. Utilization of this vector provides high levels of protein expression. (B) Real time PCR amplification plot using a custom designed probe which amplifies the CMV region of the CLS construct.

The CRLS construct was placed under the control of the human cytomegalovirus immediate-early enhancer and chicken $\beta$-actin promoter with first intron. This vector allows for high levels of protein expression (9) in all tissues, which would be beneficial for studies using heart, skeletal or liver tissue (8). Interestingly, Kiebish et al. also developed a mouse line that overexpressed CRLS; however, the transgene was heart specific (7). Although Kiebish et al. did not show restored total cardiolipin levels during a 
type 1 diabetic insult as one would expect, the authors determined that tetralineolic cardiolipin levels were restored back to normal levels, which ultimately attenuated the mitochondrial dysfunction associated with type 1 diabetes mellitus.

Because CRLS is nuclear-encoded, another strategy that could potentially result in increased CRLS protein content is to increase protein import rates. During type 1 diabetes mellitus, protein import rates are decreased, specifically in the diabetic IFM. By restoring protein import rates, more CRLS could enter mitochondria and potentially improve cardiolipin content, ultimately preserving mitochondrial function. By utilizing the novel transgenic mouse line described in this dissertation (Chapter 4), future studies can examine the therapeutic benefit of Grp75 overexpression on protein import rates to determine if the import rates are rescued, which in turn could restore CRLS and other proteins altered during a type 1 diabetic insult.

Increased ROS production is the main contributor to mitochondrial dysfunction associated with diabetes mellitus; therefore, prevention of an enhanced oxidative environment may lead to preservation of the IMM. For example, animal models manipulated to overexpress players involved in the antioxidant defense system, such as manganese superoxide dismutase, have provided protection against mitochondrial dysfunction associated with diabetes mellitus (10). Our laboratory recently reported that overexpression of mitochondrial phospholipid hydroperoxide glutathione peroxidase, which is involved in the antioxidant defense system of mitochondria, restores mitochondrial function during type 1 diabetes mellitus through scavenging lipid 
hydroperoxides (1). This model specifically could also be beneficial to the cardiolipin environment within the IMM, by potentially restoring cardiolipin levels and ultimately, mitochondrial function during diabetes mellitus.

Other than cardiolipin, phospholipid alterations to phospholipids housed within in the IMM could restore functionality to a damaged IMM. For example, cardiac mitochondrial PG content has also shown to be decreased during type 1 diabetes mellitus (6). By creating a transgenic mouse line that overexpresses a constituent in the biosynthetic pathway of PG, the decreased PG content may be restored. Not only would this restoration aid in the preservation of the IMM, but PG, along with CDP-DAG, is also hydrolyzed by CRLS to produce cardiolipin; therefore, cardiolipin levels could also be restored. Other phospholipids housed within the IMM include, but are not limited to, phosphatidylcholine, phosphatidylserine, and phosphatidyl-ethanolamine. If damaged during diabetes mellitus, restoration of any of the phospholipids that compose the IMM would be beneficial to the IMM environment and subsequent function.

The goal of this dissertation was to evaluate the impact of type 1 diabetes mellitus on the IMM phospholipid environment, specifically cardiolipin, and the processes that are housed within. The overall conclusion highlighted the importance of preserving the IMM; therefore, future directions should focus on this preservation through further examination of the impact of diabetes mellitus to the mitochondrial proteome and processes, in order to attenuate the mitochondrial dysfunction that is associated with diabetes mellitus. 


\subsection{References}

1. Baseler WA, Dabkowski ER, Jagannathan R, Thapa D, Nichols CE, Shepherd DL, Croston TL, Powell M, Razunguzwa TT, Lewis SE, Schnell DM, and Hollander JM. Reversal of mitochondrial proteomic loss in Type 1 diabetic heart with overexpression of phospholipid hydroperoxide glutathione peroxidase. Am J Physiol Regul Integr Comp Physiol 304: R553-565, 2013.

2. Boudina S and Abel ED. Mitochondrial uncoupling: a key contributor to reduced cardiac efficiency in diabetes. Physiology (Bethesda) 21: 250-258, 2006.

3. Bugger H and Abel ED. Rodent models of diabetic cardiomyopathy. Dis Model Mech 2: 454-466, 2009.

4. Dabkowski ER, Baseler WA, Williamson CL, Powell M, Razunguzwa TT, Frisbee JC, and Hollander JM. Mitochondrial dysfunction in the type 2 diabetic heart is associated with alterations in spatially distinct mitochondrial proteomes. Am J Physiol Heart Circ Physiol 299: H529-540, 2010.

5. Dabkowski ER, Williamson CL, Bukowski VC, Chapman RS, Leonard SS, Peer CJ, Callery PS, and Hollander JM. Diabetic cardiomyopathy-associated dysfunction in spatially distinct mitochondrial subpopulations. Am J Physiol Heart Circ Physiol 296: H359-369, 2009.

6. Han X, Yang J, Cheng H, Yang K, Abendschein DR, and Gross RW. Shotgun lipidomics identifies cardiolipin depletion in diabetic myocardium linking altered substrate utilization with mitochondrial dysfunction. Biochemistry 44: 16684-16694, 2005. 
7. Kiebish MA, Yang K, Sims HF, Jenkins CM, Liu X, Mancuso DJ, Zhao Z, Guan S, Abendschein DR, Han X, and Gross RW. Myocardial Regulation of Lipidomic Flux by Cardiolipin Synthase: SETTING THE BEAT FOR BIOENERGETIC EFFICIENCY. J Biol Chem 287: 25086-25097, 2012.

8. Lu B, Xu FY, Jiang YJ, Choy PC, Hatch GM, Grunfeld C, and Feingold KR. Cloning and characterization of a cDNA encoding human cardiolipin synthase (hCLS1). J Lipid Res 47: 1140-1145, 2006.

9. Niwa H, Yamamura K, and Miyazaki J. Efficient selection for high-expression transfectants with a novel eukaryotic vector. Gene 108: 193-199, 1991.

10. Shen X, Zheng S, Metreveli NS, and Epstein PN. Protection of cardiac mitochondria by overexpression of MnSOD reduces diabetic cardiomyopathy. Diabetes 55: 798-805, 2006. 


\section{Chapter 6:}

Appendix 
Figure 6.1

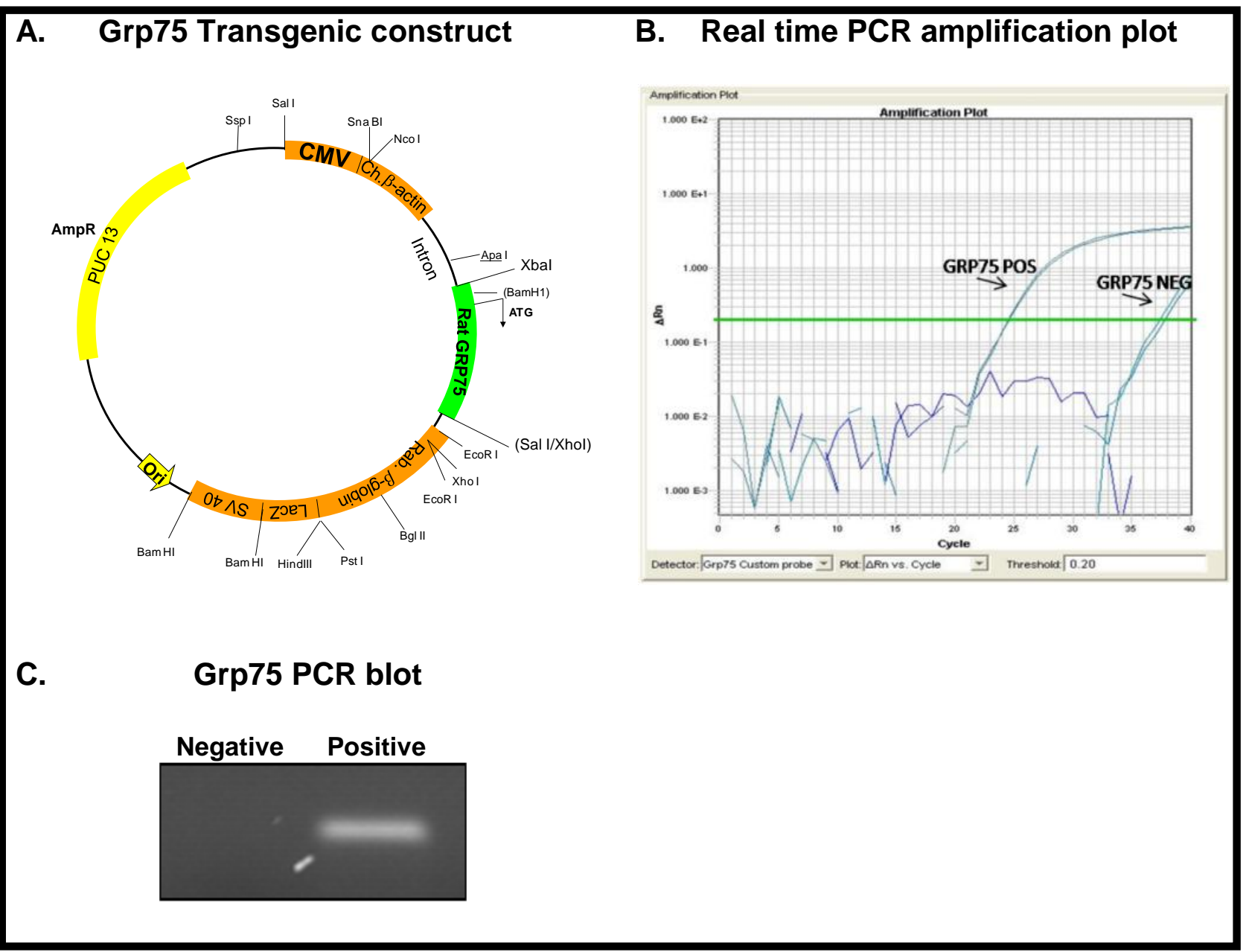


Figure 6.1. Grp75 vector map. (A.) Map of GRP75 transgenic construct housed in a pCAGGS vector. (B.) Real time PCR amplification plot using a custom designed probe which amplifies the CMV region of the Grp75 construct. (C.) PCR analysis was also performed to confirm transgene presence in positive mice. $N=2$. 
Figure 6.2

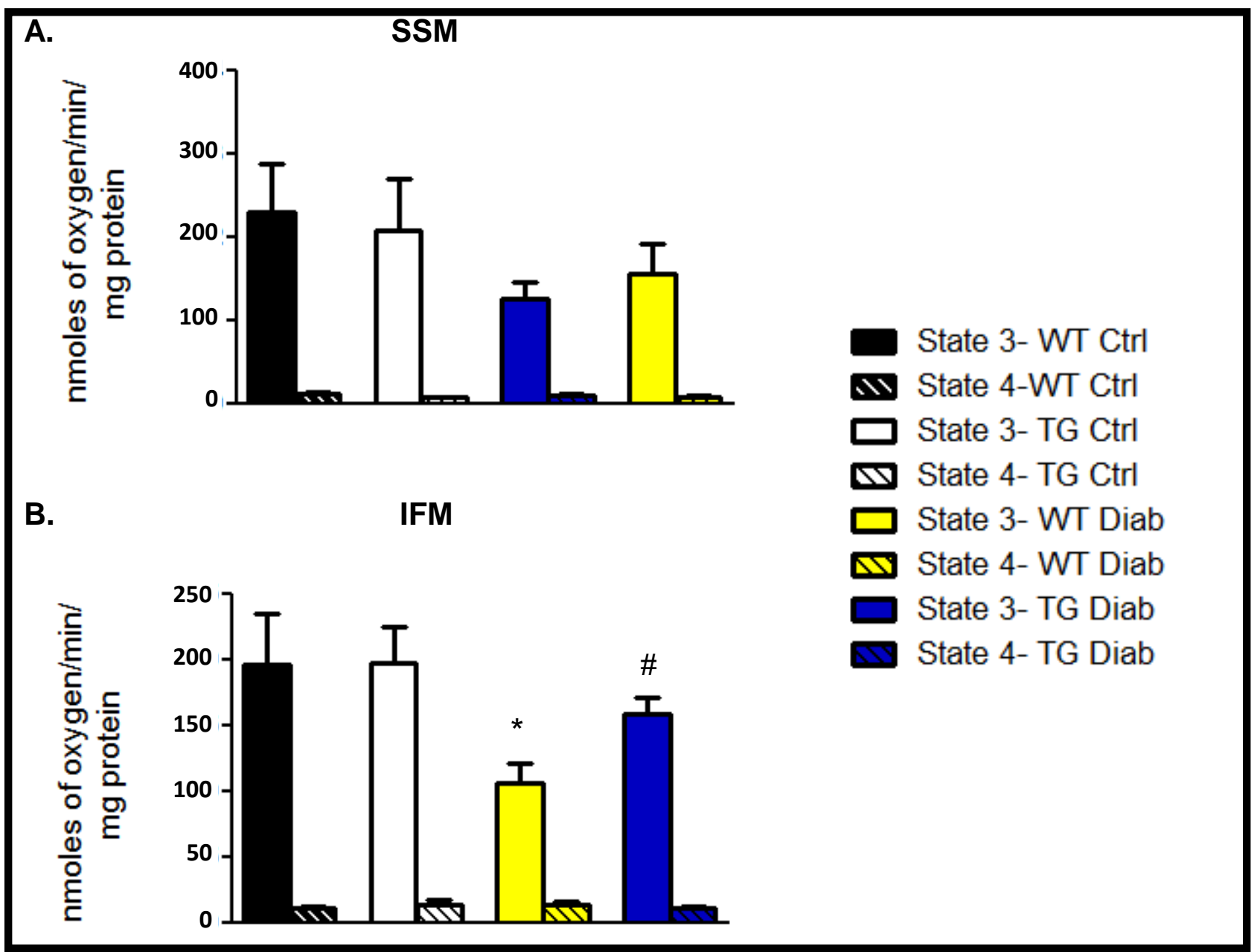


Figure 6.2. The protective benefit of Grp75 overexpression on mitochondrial respiration. Mitochondria respiration measured in isolated mitochondria from wild-type control (WT Ctrl), Grp75 transgenic control (TG Ctrl), wild-type diabetic (WT Diab) and transgenic diabetic (TG Diab). (A.) SSM state 3 and state 4 respiration measured in all four groups. (B.) IFM state 3 and state 4 respiration measured in all four groups. Key is included. Values are presented as means \pm SEM; ${ }^{*} \mathrm{P} \leq 0.05$ WT Diab vs WT Ctrl. \#P $\leq$ 0.05 WT Diab vs TG Diab; $N=7$ control wild-type control, 6 Grp75 transgenic, 9 wildtype diabetic and 6 transgenic diabetic. 
Figure 6.3

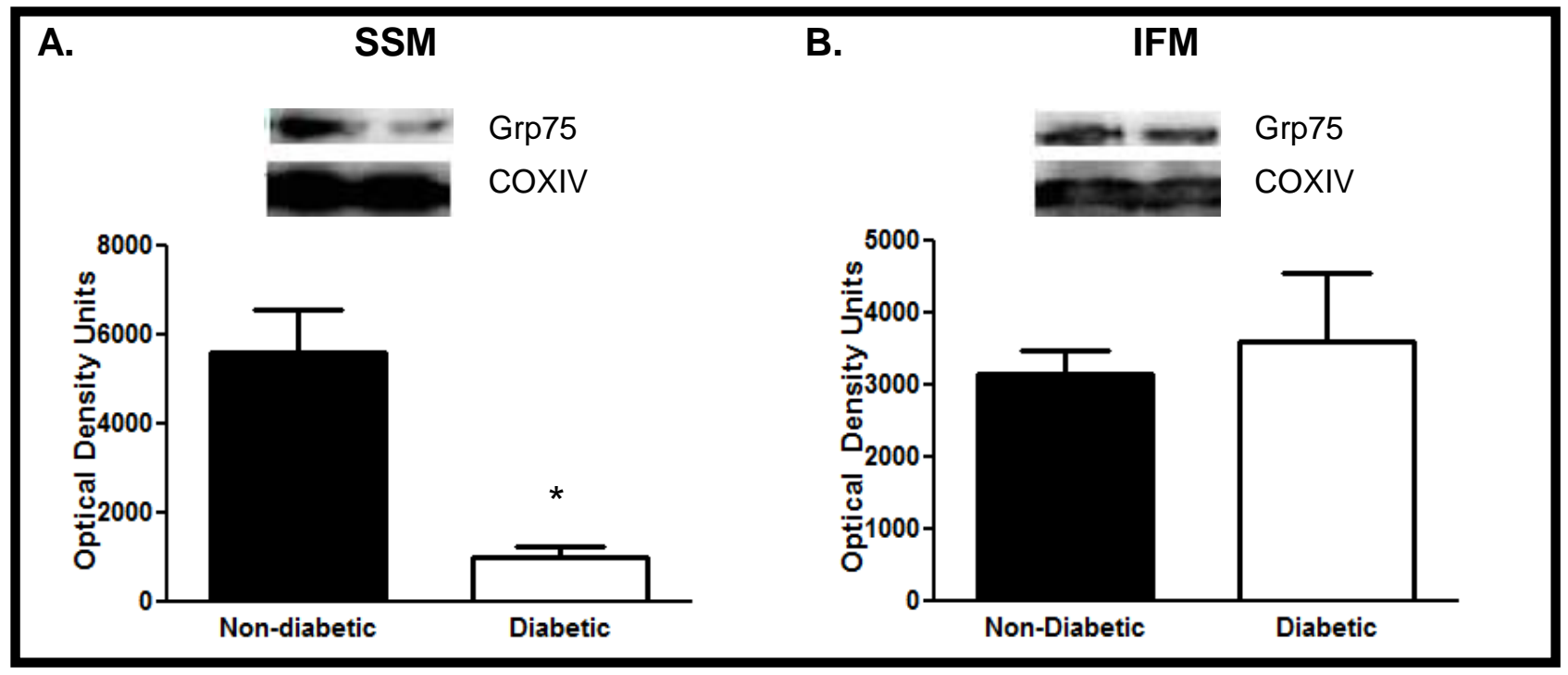


Figure 6.3. Protein levels of Grp75 in the type 2 diabetic human heart. Western blot analysis of protein import constituent Grp75 in non-diabetic and diabetic human heart tissue. (A.) Representation of Grp75 protein content in SSM and in (B.) IFM. Control for protein loading was confirmed by COXIV. Values are presented as means $\pm \mathrm{SEM}$; ${ }^{*} \mathrm{P}<$ 0.05 Non-diabetic vs. diabetic; $N=4$. 
Figure 6.4

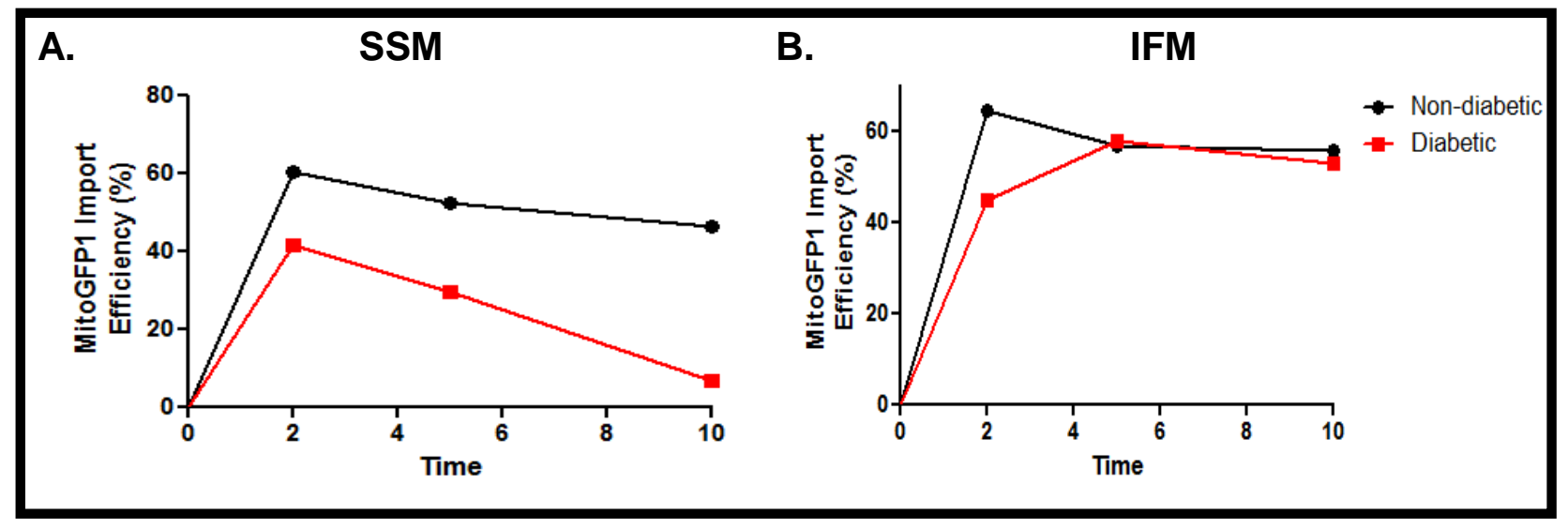


Figure 6.4. Preliminary protein import in the type 2 diabetic human heart. (A.) Protein import rates between non-diabetic and diabetic human SSM. (B.) Protein import rates between non-diabetic and diabetic human IFM. Values are presented as percent of control. $N=1$. 
Figure 6.5

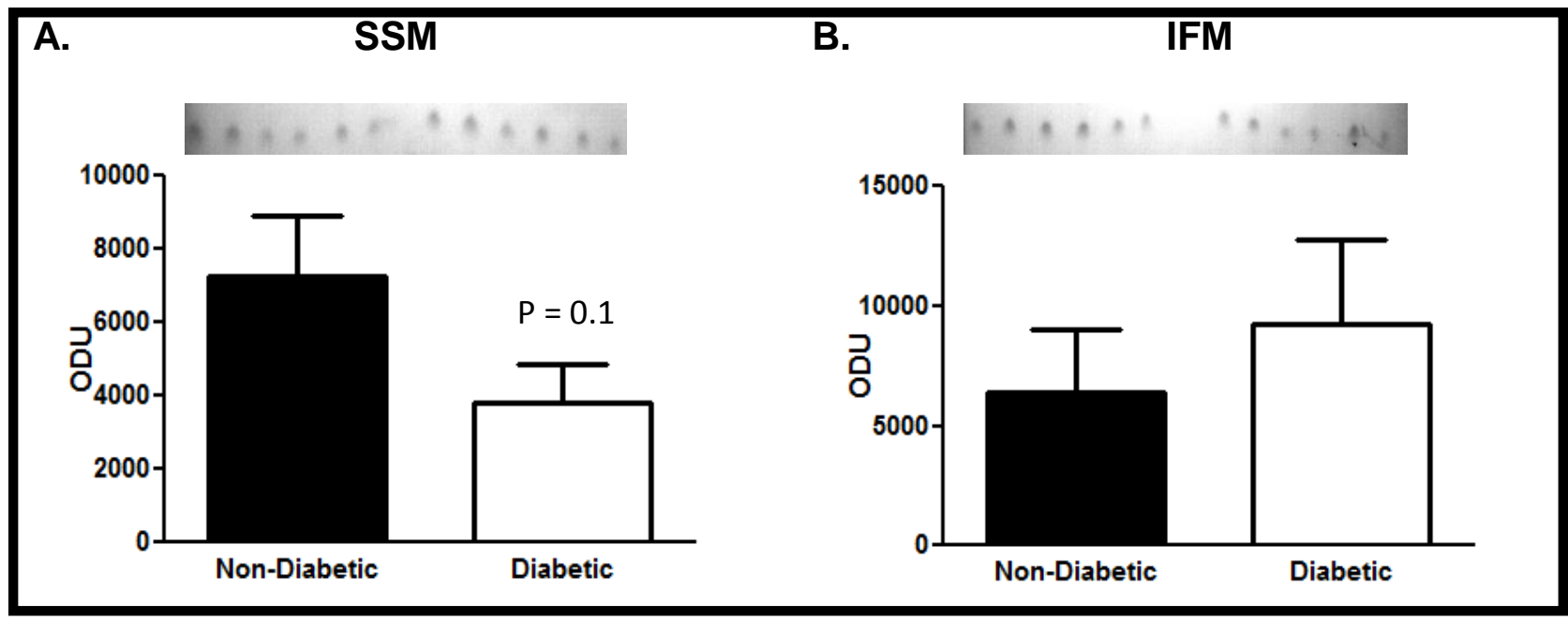


Figure 6.5. Cardiolipin content in the type 2 diabetic heart. High performance thin layer chromatography of cardiolipin content in isolated mitochondrial subpopulations of non-diabetics and type 2 diabetic patients. (A.) Representation of cardiolipin content in SSM and in (B.) IFM. Values are presented as means \pm SEM. Two spots represent one sample; $N=3$. 
Table 6.1

\begin{tabular}{|l|c|}
\hline \multicolumn{2}{|c|}{$\begin{array}{c}\text { Mean Fluorescence } \\
\text { Intensity }\end{array}$} \\
\hline SSM & \\
\hline Control & 36.77 \\
\hline Transgenic Grp75 & 39.41 \\
\hline IFM & \\
\hline Control & 42.42 \\
\hline Transgenic Grp75 & 45.92 \\
\hline
\end{tabular}


Table 6.1. Grp75 overexpression in isolated mitochondrial subpopulations. Mitochondrial subpopulations were incubated with MitoTracker Deep Red 633 and Grp75-PE and subjected to flow cytometry. Mitochondria that were co-stained with both dyes were analyzed. The control mean fluorescence intensity was compared to the transgenic mean fluorescence intensity for each subpopulation. $N=1$. 


\section{CURRICULUM VITAE}

\section{Tara L. Croston}

\section{OFFICE ADDRESS}

Division of Exercise Physiology

Center for Cardiovascular and Respiratory Sciences

West Virginia School of Medicine

P.O. Box 9227

Morgantown, WV 26506

Tel: (304) 293-7311; Fax: (304) 293-7105

Email: tcroston@hsc.wvu.edu

\section{EDUCATION}

West Virginia University (Morgantown, WV)

2008 - Present

School of Medicine; Division of Exercise Physiology

Center for Cardiovascular and Respiratory Sciences

Biomedical Health Sciences; Ph.D. Candidate

2012 - Present

Frostburg State University (Frostburg, MD)

$2002-2007$

Bachelor of Science in Biology/Secondary Education

Chemistry minor 


\section{Dissertation}

The Diabetic Heart: Examination of the Inner Mitochondrial Membrane

Mentor: Dr. John Hollander

Committee Members: Stephen Alway, S. Jamal Mustafa, Emidio Pistilli, and Mark Olfert

\section{HONORS and AWARDS}

Selected speaker to present at E.J. Van Liere Memorial Convocation

and HSC Research Day; WVU

Selected speaker from West Virginia University to present at

Appalachian Regional Cell Conference; WVU

Appointed trainee on the NIH Predoctoral Training Grant in

$2010-2012$

Cardiovascular and Pulmonary Diseases (T-32); WVU

E.J. Van Liere Memorial Research Convocation: $2^{\text {nd }}$ Place

2010

Poster Presentation; WVU

\section{PROFESSIONAL SOCIETIES}

Member, American Physiology Society; WVU

Member, American Heart Association; WVU

Member, American College of Sports Medicine; WVU

Member, Beta Beta Beta National Biology Honor Society; FSU

President, Beta Beta Beta National Biology Honor Society; FSU

Member, American Chemical Society; FSU
2010 - Present
$2011-2012$
$2010-2011$
$2004-2007$
$2005-2007$
$2004-2007$ 


\section{RESEARCH SUPPORT}

WVU School of Medicine Predoctoral Fellowship

2012 - Present

$2008-2010$

AHA Predoctoral Fellowship (Submitted January 2012; not funded)

2012

Average Priority Score:

1.81

Percentile Rank:

$23.96 \%$

NIH T-32 Pre-doctoral Training Grant (5T32HL090610)

$2010-2012$

Appointed trainee member on the NIH T32 Training Grant in cardiovascular and pulmonary diseases

Role: Trainee

\section{PRESENTATIONS}

Croston TL, Holden AA, Tveter K, and Hollander JM. Is the type 2 diabetic mouse heart a good predictor for the diabetic human population? Van Liere Convocation, West Virginia University. 2013

Croston TL, Shepherd DL, Baseler WA, Dabkowski ER, Thapa D, Nichols CE, Jagannathan R, Lewis SE, Hollander JM. Cardiolipin impacts mitochondrial functional integrity in diabetic cardiomyopathy. Appalachian Regional Cell Conference. 2012 
Croston TL, Shepherd DL, Baseler WA, Thapa D, Nichols CE, Hollander JM. Examination of cardiolipin biosynthesis in the diabetic heart. FASEB J. 2012 26:Ib746

Croston TL, Shepherd DL, Baseler WA, Dabkowski ER, Thapa D, Nichols CE, Jagannathan R, Lewis SE, Hollander JM. Influence of diabetic cardiomyopathy on cardiolipin biosynthesis. Van Liere Convocation, West Virginia University. 2012

Croston TL, Baseler, WA, Thapa D, Lewis SE, Nurkiewicz TR, Yi J, Hollander JM. Influence of Nanoparticle Inhalation on Cardiac Mitochondrial Function FASEB J. 2011 25:Ib561

Croston TL, Hollander JM. Examination of Cardiolipin Synthase in the Diabetic Heart. CCRS Retreat. 2011

Croston TL, Baseler, WA, Thapa D, Nurkiewicz TR, Hollander JM. Examination of Mitochondrial Function Influenced by Environmental Nanoparticle Exposure. Van Liere Convocation, West Virginia University. 2011

Croston TL, Williamson CL, Dabkowski ER, Baseler WA, Hollander JM. Heat Shock Protein 27 Overexpression Protects against Diabetic Cardiomyopathy through a Mitochondria-associated Mechanism. Van Liere Convocation, West Virginia University. 2010

Croston TL, Dabkowski ER, Williamson .L, Baseler WA, Hollander JM. Mitochondrial subpopulationspecific proteomic alterations in the type 2 diabetic heart. FASEB J. 2010 24:Ib573 


\section{PUBLICATIONS}

\section{Articles}

Croston TL, Lewis SE, Thapa D, Shepherd DL, Nichols CE, Jagannathan R, Hollander JM. Mitochondrial dysfunction in diabetic human heart: impact on distinct mitochondrial subpopulations. 2013 (In preparation)

Croston TL, Shepherd DL, Thapa D, Nichols CE, Lewis SE, Dabkowski ER, Jagannathan R, Baseler WA, Hollander JM. Evaluation of the cardiolipin biosynthetic pathway and its interactions in the diabetic heart. 2013 Life Sci. 2013 (doi: 10.1016/j.Ifs.2013.07.005)

Shepherd DL, Croston TL, Nichols CE, Thapa D, Baseler WA, Lewis SE, Hollander JM. Longitudinal assessment of type I diabetes mellitus using conventional echocardiography and speckle-tracking based strain imaging. 2013 (In preparation)

Jagannathan R, Thapa D, Baseler W, Shepherd D, Croston T, Nichols C, Lewis S, Hollander J. Translational Regulation of the Mitochondrial Genome Following Redistribution of Mitochondrial MicroRNA (MitomiR) in the Diabetic Heart. Cell Metabolism. 2012 (Submitted)

Baseler W, Thapa D, Jagannathan R, Dabkowski ER, Croston TL, Hollander JH. miR-141 as a Regulator of the Mitochondrial Phosphate Carrier (SIc25a3) in the Type 1 Diabetic Heart. Am J Physiol Cell Physiol. 2012 (PMID:23034391) 
Baseler W, Dabkowski ER, Jagannathan R, Thapa D, Nichols CE, Shepherd DL, Croston TL, Powell M, Razunguzwa TT, Lewis SE, Schnell DM, Hollander JH. Reversal of Mitochondrial Proteomic Loss in Type 1 Diabetic Heart with Overexpression of Phospholipid Hydroperoxide Glutathione Peroxidase. Am J Physiol Regul Integr Comp Physiol. 2012 [Epub ahead of print]

Baseler WA, Croston TL, Hollander JM. Mortalin Biology- Stress, Life and Death. Chapter 4: Functional Characteristics of Mortalin. Springer Science, (2012). ISBN 978-94-007-3026-7

Baseler WA, Dabkowski ER, Williamson CL, Croston TL, Thapa D, Powell MJ, Razunguzwa TT, Hollander JM. Proteomic alterations of distinct mitochondrial subpopulations in the type 1 diabetic heart: contribution of protein import dysfunction. Am J Physiol Regul Integr Comp Physiol. 2011 Feb;300(2):R186-200. Epub 2010 Nov 3. PMID: 21048079

Williamson CL, Dabkowski ER, Baseler WA, Croston TL, Alway SE, Hollander JM. Enhanced apoptotic propensity in diabetic cardiac mitochondria: influence of subcellular spatial location. Am J Physiol Heart Circ Physiol. 2010 Feb;298(2):H633-42. Epub 2009 Dec 4. PMID: 19966057

\section{Abstracts}

Croston TL, Holden AA, Tveter K, and Hollander JM. Is the type 2 diabetic mouse heart a good predictor for the diabetic human population? Van Liere Convocation, West Virginia University. 2013. 
Thapa D, Jagannathan R, Croston TL, Hollander JM. Novel interaction of mitofilin with $F_{0}-A T P$ synthase subunits in cardiac subpopulations. Van Liere Convocation, West Virginia University. 2013.

Shepherd DL, Croston TL, Lewis SE, Nichols CE, Thapa D, Jagannathan R, Hollander JM. Mitochondrial Translocation and Phosphorylation: What's the Hsp27 connection? Van Liere Convocation, West Virginia University. 2013.

Croston TL, Holden AA, Tveter KJ, Lewis SE, Thapa D, Shepherd DL, Nichols CE, Jagannathan R, Hollander JM. Diabetic mouse hearts: a good predictor for the human population? FASEB J. 2013, Abstract \#1668

Fancher IS, Croston TL, Shepherd DL, Dick GM, Hollander JM. Cardiac interfibrillar mitoK ATP $_{\text {channels are }}$ sensitive to diazoxide and have a regulatory subunit profile distinct from subsarcolemmal mitoK ATP. $_{\text {. }}$

FASEB J. 2013

Lewis SE, Dabkowski ER, Baseler WA, Shepherd DL, Croston TL, Thapa D, Nichols CE, Hollander JM. Impact of mitochondria phospholipid hydroperoxide glutathione peroxidase (mPHGPx) overexpression on the type 1 diabetic heart. FASEB J. 2013, Abstract \#1670 
Nichols CE, Shepherd DL, Croston TL, Thapa D, Lewis SE, Jagannathan R, Yi J, Nurkiewicz TR, Hollander JM. Acute Inhalation Exposure of Nano-Titanium Dioxide induces Cardiac and Mitochondrial Dysfunction in Mice. FASEB J. 2013, Abstract \#1679

Thapa D, Jagannathan R, Croston TL, Baseler WA, Nichols CE, Shepherd DL, Lewis SE, Hollander JM. Interaction of mitofilin with respiratory complexes in mitochondrial subpopulations. FASEB J. 2013, Abstract \#1669

Croston TL, Shepherd DL, Baseler WA, Dabkowski ER, Thapa D, Nichols CE, Jagannathan R, Lewis SE, Hollander JM. Cardiolipin impacts mitochondrial functional integrity in diabetic cardiomyopathy. Appalachian Regional Cell Conference. 2012

Nichols CE, Croston TL, Shepherd DL, Thapa D, LaFata GL, Lewis SE, Knuckles TL, McCawley M, Hendryx M, Nurkiewicz TR, Hollander JM. Mountain-top mining particulate exposure decreases function and increases apoptotic propensity of distinct subcellular species of cardiac mitochondria. Appalachian Regional Cell Conference. 2012

Shepherd DL, Croston TL, Lewis SE, Nichols CE, Thapa D, Jagannathan R, Hollander JM. Mitochondrial Translocation of Heat Shock Protein 27 (hsp27) Protects Against Diabetic Cardiomyopathy. Appalachian Regional Cell Conference. 2012 
Thapa D, Baseler WA, Jagannathan R, Dabkowski ER, Croston TL, Nichols CE, Shepherd DL, Lewis SE, Hollander JM. Cardiomyocytes enrichment with miR-141 regulates ATP content. Appalachian Regional Cell Conference. 2012

Croston TL, Shepherd DL, Baseler WA, Thapa D, Nichols CE, Hollander JM. Examination of cardiolipin biosynthesis in the diabetic heart. FASEB J. 2012 26:Ib746

Baseler WA, Dabkowski ER, Jagannathan R, Thapa D, Nichols CE, Shepherd DL, Croston TL, Schnell DM, Hollander JM. Overexpression of phospholipid hydroperoxide glutathione peroxidase (MPHGPX) attenuates cardiac mitochondrial proteomic loss and reverses protein import detriments observed with type 1 diabetes mellitus. FASEB J. 2012 26:1127.4

Fancher IS, Baseler WA, Croston TL, Thapa D, Shepherd DL, Nichols CE, Lewis SE, Jagannathan R, Asano S, Dick GM, Hollander JM. Differential expression of mitoKATP subunits in cardiac mitochondrial subpopulations and the influence of Type I diabetes. FASEB J. 2012 26:1057.6

Lewis SE, Baseler WA, Croston TL, Thapa D, Jagannathan R, Hollander JM. Type 1 diabetes mellitus differentially regulates mitochondrially-encoded proteins in cardiac mitochondrial subpopulations. FASEB J. 2012 26:Ib748 
Jagannathan R, Baseler WA, Thapa D, Croston TL, Shepherd DL, Nichols CE, Hollander JM. HDAC6 regulates mitochondrial oxidative phosphorylation by ATP synthase $\beta$ subunit acetylation in diabetic cardiomyopathy. FASEB J. 2012 26:869.13

Nichols CE, Baseler WA, Thapa D, LaFata GL, Croston TL, Shepherd DL, Lewis SE, Knuckles TL, McCawley M, Hendryx M, Nurkiewicz TR, Hollander JM. Mountain-top mining particulate matter exposure increases markers of mitochondrially-driven apoptosis in rat cardiac tissue. FASEB J. 2012 26:1036.15

Shepherd DL, Croston TL, McLaughlin SL, Baseler WA, Nichols CE, Thapa D, Lewis SE, Hollander JM. Longitudinal assessment of type I diabetes mellitus using conventional echocardiography and speckletracking based strain imaging. FASEB. 2012 26:1054.11

Thapa D, Baseler WA, Jagannathan R, Dabkowski ER, Croston TL, Nichols CE, Shepherd DL, Lewis SE, Hollander JM. miRNA-141 is a potential regulator of the mitochondrial phosphate carrier (slc25a3) in the type 1 diabetic heart. FASEB J. 2012 26:869.11

Croston TL, Shepherd DL, Baseler WA, Dabkowski ER, Thapa D, Nichols CE, Jagannathan R, Lewis SE, Hollander JM. Influence of diabetic cardiomyopathy on cardiolipin biosynthesis. Van Liere Convocation, West Virginia University. 2012 
Shepherd DL, Croston TL, Nichols CE, Baseler WA, Thapa D, Hollander JM. Conventional echocardiography and speckle-tracking based strain imaging of the type 1 diabetic heart. Van Liere Convocation, West Virginia University. 2012

Croston TL, Baseler WA, Thapa D, Lewis SE, Nurkiewicz TR, Yi J, Hollander JM. Influence of Nanoparticle Inhalation on Cardiac Mitochondrial Function. FASEB J. 2011 25:Ib561

Baseler WA, Dabkowski ER, Thapa D, Croston TL, Williamson CL, Hollander JM. Proteomic alterations of distinct mitochondrial subpopulations in the type 1 diabetic heart: Contribution of protein import dysfunction. FASEB J. 2011 25: 825.6

Dabkowski ER, Baseler WA, Croston TL, Thapa D, Hollander JM. Mitochondrial phospholipid hydroperoxide glutathione peroxidase (mPHGPx) overexpression preserves the inner mitochondrial membrane in the diabetic heart. FASEB J. 2011 25:1095.5

Thapa D, Baseler WA, Croston TL, Lewis SE, Hollander JM. Examination of microRNA (miRNA) dysregulation in the type 1 diabetic heart and its functional implications. FASEB J. 2011 25:Ib464 
Croston TL, Baseler WA, Thapa D, Nurkiewicz TR, Hollander JM. Examination of Mitochondrial Function Influenced by Environmental Nanoparticle Exposure. Van Liere Convocation, West Virginia University. 2011

Thapa D, Baseler WA, Croston TL, Lewis SE, Hollander JM. MicroRNA Dysregulation and Functional Implications on Mitochondrial Proteomes in Type 1 Diabetic Heart. Van Liere Convocation, West Virginia University. 2011

Croston TL, Dabkowski ER, Williamson CL, Baseler WA, Hollander JM. Mitochondrial subpopulationspecific proteomic alterations in the type 2 diabetic heart. FASEB J. 2010 24:Ib573

Baseler WA, Williamson CL, Dabkowski ER, Croston TL, Hollander JM. Mitochondria-specific overexpression of phospholipid hydroperoxide glutathione peroxidase (GPx4) attenuates ischemia/reperfusion (I/R) associated apoptosis. FASEB J. 2010 24:Ib560

Dabkowski ER, Baseler WA, Croston TL, Williamson CL, Hollander JM. Mitochondrial Overexpression of Phospholipid Hydroperoxide Glutathione Peroxidase 4 (mPHGPx) Provides Cardioprotection From Type 1 Diabetes Mellitus Insult. FASEB J. 2010 24:789.2 
Croston TL, Williamson CL, Dabkowski ER, Baseler WA, Hollander JM. Heat Shock Protein 27

Overexpression Protects against Diabetic Cardiomyopathy through a Mitochondria-associated Mechanism. Van Liere Convocation, West Virginia University. 2010

\section{$\underline{\text { References }}$}

Dr. John Hollander; Director of Graduate Studies and Associate Professor

(304) 293-3683

of Exercise Physiology; PhD Mentor

jhollander@hsc.wvu.edu

Dr. Stephen Alway; Professor and Chair of Exercise Physiology

(304) 293-0772

salway@hsc.wvu.edu

Dr. S. Jamal Mustafa; Professor of Physiology and Pharmacology

(304) 293-5830

and Assistant VP for Research

smustafa@hsc.wvu.edu 